\title{
Modality for Scenario Analysis and Maximum Likelihood Allocation
}

\author{
Takaaki Koike* and Marius Hofert ${ }^{\dagger}$
}

April 28, 2022

\begin{abstract}
We study the variability of a risk from the statistical viewpoint of multimodality of the conditional loss distribution given that the aggregate loss equals an exogenously provided capital. This conditional distribution serves as a building block for calculating risk allocations such as the Euler capital allocation of Value-at-Risk. A superlevel set of this conditional distribution can be interpreted as a set of severe and plausible stress scenarios the given capital is supposed to cover. We show that various distributional properties of this conditional distribution, such as modality, dependence and tail behavior, are inherited from those of the underlying joint loss distribution. Among these properties, we find that modality of the conditional distribution is an important feature in risk assessment related to the variety of risky scenarios likely to occur in a stressed situation. Under unimodality, we introduce a novel risk allocation method called maximum likelihood allocation (MLA), defined as the mode of the conditional distribution given the total capital. Under multimodality, a single vector of allocations can be less sound. To overcome this issue, we investigate the so-called multimodalty adjustment to increase the soundness of risk allocations. Properties of the conditional distribution, MLA and multimodality adjustment are demonstrated in numerical experiments. In particular, we observe that negative dependence among losses typically leads to multimodality, and thus a higher multimodality adjustment can be required.
\end{abstract}

JEL classification: C02, G32

Keywords: Risk allocation, Scenario analysis, Variability measure, Conditional distribution, Unimodality, Mode

\section{Introduction}

Risk allocation concerns the quantification of the risk of each unit of a portfolio. For a $d$-dimensional portfolio of risks or losses represented by an $\mathbb{R}^{d}$-valued random vector $\boldsymbol{X}=\left(X_{1}, \ldots, X_{d}\right), d \in \mathbb{N}$, the overall loss $S=X_{1}+\cdots+X_{d}$ is covered by a total capital $K \in \mathbb{R}$, which is typically determined as $K=\varrho(S)$

\footnotetext{
${ }^{*}$ Corresnponding author: Department of Statistics and Actuarial Science, University of Waterloo, Waterloo, ON, Canada, E-mail: tkoike@uwaterloo.ca

${ }^{\dagger}$ Department of Statistics and Actuarial Science, University of Waterloo, Waterloo, ON, Canada, E-mail: marius.hofert@uwaterloo.ca
} 
for a risk measure $\rho$. The Euler principle, proposed in Tasche (1995), is one of the most well-known rules of risk allocation. It is economically justified, for example, in Tasche (1995) and Kalkbrener (2005), and the derived allocated capital is also known as the Aumann-Shapley value (Aumann and Shapley, 2015) in cooperative game theory; see Denault (2001).

The Euler principle is applicable when the total capital is determined by a risk measure via $K=\varrho(S)$. However, as pointed out by Asimit et al. (2019), the total capital in practice may not always coincide with the risk measure itself but includes various adjustments such as stress scenarios and liquidity adjustments. In such cases, the capital does not possess the original meaning as a risk measure and the formula under the Euler principle is not straightforwardly applicable. In addition, there are situations when the total capital is given exogenously as a constant; see Laeven and Goovaerts (2004). For the case when the total capital is regarded as a constant, various allocation methods have been proposed in the literature. One of the main streams found, for example, in Laeven and Goovaerts (2004) and Dhaene et al. (2012), is to derive an allocation as a minimizer of some loss function over a set of allocations $\mathcal{K}_{d}(K)=\left\{\boldsymbol{x} \in \mathbb{R}^{d}: x_{1}+\cdots+x_{d}=K\right\}$. Another method is to find a confidence level for which the corresponding risk measure coincides with $K$, and then allocate $K$ by regarding it as measured by a risk measure. For example, if Value-at-Risk (VaR) or Expected Shortfall (ES) are chosen as risk measures, confidence levels $p_{\mathrm{VaR}}, p_{\mathrm{ES}} \in(0,1)$ are first found such that $K=\operatorname{VaR}_{p_{\mathrm{VaR}}}(S)$ or, respectively, $K=\operatorname{ES}_{p_{\mathrm{ES}}}(S)$ hold for a given total capital $K$. After performing this procedure, the Euler principle becomes applicable and the resulting allocated capital of $K$ to the $j$ th risk $X_{j}$ is $\mathbb{E}\left[X_{j} \mid\{S=K\}\right]$ or, respectively, $\mathbb{E}\left[X_{j} \mid\left\{S \geq \operatorname{VaR}_{p_{\mathrm{ES}}}(S)\right\}\right]$; see Section 2.1 for details.

Although these methods provide plausible risk allocations, they sometimes ignore important distributional properties of $\boldsymbol{X}$ related to the soundness of risk allocations and to risky scenarios expected to be covered by the allocated capitals. As we will see in Section 2.2, most allocation methods provide the homogeneous allocation $(K / d, \ldots, K / d)$ when $\boldsymbol{X}$ is exchangeable in the sense that $\boldsymbol{X} \stackrel{\mathrm{d}}{=}\left(X_{\pi(1)}, \ldots, X_{\pi(d)}\right)$ for any permutation $(\pi(1), \ldots, \pi(d))$ of $\{1, \ldots, d\}$. This homogeneous allocation can be sound when the conditional distribution of $\boldsymbol{X}$ in a stressed situation is unimodal with the mode $(K / d, \ldots, K / d)$ since this homogeneous allocation covers the risky scenario most likely to occur in a stressed situation. On the other hand, the same allocation $(K / d, \ldots, K / d)$ is derived when the conditional distribution in a stressed situation is multimodal and $(K / d, \ldots, K / d)$ is supposed to cover multiple risky scenarios on average. In this multimodal case, the homogeneous allocation can be less sound than in the former unimodal case since multiple risky scenarios are hidden in a single vector of allocations. Therefore, the soundness of risk allocations depends on the distributional properties of the conditional distribution of $\boldsymbol{X}$ in a stressed situation. In the multimodal case, imposing a multimodality loading to the capital $K$ can be required to take the variability of scenarios into account.

In this paper, we study the variability of a risk from the statistical viewpoint of multimodality of the conditional distribution of $\boldsymbol{X}$ given $\{S=K\}$. Since $\boldsymbol{X} \mid\{S=K\}$ takes values in $\mathcal{K}_{d}(K)$, this random vector serves as a building block for deriving risk allocations. For example, the Euler allocation of $\operatorname{VaR}_{p}(S), p \in(0,1)$, arises as the expectation of $\boldsymbol{X} \mid\{S=K\}$ with $K=\operatorname{VaR}_{p}(S)$. In addition, we show in Section 2.2 that a superlevel set of $\boldsymbol{X} \mid\{S=K\}$ can be regarded as a set of severe and plausible stress scenarios the given capital $K$ is supposed to cover. Based on the motivation provided there, we investigate distributional properties of $\boldsymbol{X} \mid\{S=K\}$ in Section 3. We show that unimodality, dependence and tail behavior of $\boldsymbol{X} \mid\{S=K\}$ are typically inherited from those of the underlying unconditional loss $\boldsymbol{X}$, 
respectively. Moreover, we demonstrate in Section 5.2 that negative dependence among $\boldsymbol{X}$ typically leads to multimodality of $\boldsymbol{X} \mid\{S=K\}$. These observations can be useful to detect the hidden risk of multimodality in risk allocation. The properties of $\boldsymbol{X} \mid\{S=K\}$ studied in this paper are of potential importance in simulation and statistical inference of $\boldsymbol{X} \mid\{S=K\}$ using Markov chain Monte Carlo (MCMC) methods for efficiently simulating the distribution of interest; see Remark 2 and Appendix F.

We also propose a novel risk allocation method termed maximum likelihood allocation (MLA), which is defined as the mode of $\boldsymbol{X} \mid\{S=K\}$ assuming that it is unimodal. Besides the mean (which leads to the Euler allocation of $\mathrm{VaR}$ ), the mode is also an important summary statistics of $\boldsymbol{X} \mid\{S=K\}$. It can be interpreted as the risky scenario most likely to occur in the stressed situation $\{S=K\}$. By searching for the global mode of $\boldsymbol{X} \mid\{S=K\}$, possibly multiple local modes can be detected. As explained in Section 2.2, this procedure of detecting multimodality is beneficial for evaluating the soundness of risk allocations, for discovering hidden multiple scenarios likely to occur in the stressed situation $\{S=K\}$ and for constructing more flexible risk allocations by weighting important scenarios. In Section 4.1 we present a definition and properties of MLA expected to hold for a risk allocation. In Section 4.2 we introduce the so-called multimodality adjustment to increase the soundness of risk allocations when $\boldsymbol{X} \mid\{S=K\}$ is multimodal. The multimodality adjustment can be regarded as a measure of variability of the underlying risk; see Furman and Landsman (2006) and Furman et al. (2017) for studies of variability measures of tail risk. MLA and multimodality-adjusted allocated capitals are estimated and compared with Euler allocations in numerical experiments in Section 5. Concluding remarks are given in Section 6 and all proofs can be found in Appendix A.

\section{Preliminaries}

\subsection{A brief introduction to capital allocation}

On a standard atomless probability space $(\Omega, \mathcal{A}, \mathbb{P})$, let $\boldsymbol{X}=\left(X_{1}, \ldots, X_{d}\right), d \geq 2$ be a $d$-dimensional random vector with joint distribution function $F_{\boldsymbol{X}}$ with margins $F_{X_{1}}, \ldots, F_{X_{d}}$ and a copula $C$. Furthermore, let $S=X_{1}+\cdots+X_{d}$ and denote $F_{S}$ by its distribution function. If $F_{S}$ and $F_{\boldsymbol{X}}$ have densities, we denote them by $f_{S}$ and $f_{\boldsymbol{X}}$, respectively, with marginal densities $f_{X_{1}}, \ldots, f_{X_{d}}$ of $f_{\boldsymbol{X}}$ and copula density $c$. The variable $X_{j}$ is interpreted as loss of the $j$ th asset, business line, economic entity and so on, of the portfolio $\boldsymbol{X}$ in a fixed period of time. Similarly, $S$ is regarded as the aggregate risk of the portfolio $\boldsymbol{X}$. Positive values of $X_{1}, \ldots, X_{d}$ and $S$ are understood as losses and negative values are interpreted as profits.

The amount of total capital required to cover the risk of the portfolio $\boldsymbol{X}$ is often determined as $\varrho(S)$ where $\varrho$ is a risk measure, that is, a map from a set of random variables to a real number. Examples of risk measures include Value-at-Risk (VaR) at confidence level $p \in(0,1)$ defined by

$$
\operatorname{VaR}_{p}(X)=\inf \left\{x \in \mathbb{R}: F_{X}(x) \geq p\right\}
$$

for a random variable $X$ on $(\Omega, \mathcal{A}, \mathbb{P})$ and its distribution function $F_{X}$, and Expected Shortfall (ES) at confidence level $p \in(0,1)$, also known as Conditional VaR, Tail VaR and Average VaR, defined by

$$
\operatorname{ES}_{p}(X)=\frac{1}{1-p} \int_{p}^{1} \operatorname{VaR}_{q}(X) \mathrm{d} q
$$


provided that $\mathbb{E}[|X|]<\infty$.

Once the total capital is determined as $K \in \mathbb{R}$, it is decomposed into $d$ real numbers $\mathrm{AC}_{1}, \ldots, \mathrm{AC}_{d}$ such that the full allocation property

$$
\mathrm{AC}_{1}+\cdots+\mathrm{AC}_{d}=K
$$

holds. The set of all possible allocations is denoted by

$$
\mathcal{K}_{d}(K):=\left\{\boldsymbol{x} \in \mathbb{R}^{d}: x_{1}+\cdots+x_{d}=K\right\}
$$

If $K=\varrho(S)$ for a positive homogeneous risk measure $\varrho$, the so-called Euler principle determines the $j$ th allocated capital by

$$
\mathrm{AC}_{j}^{\text {Euler }}=\left.\frac{\partial \varrho\left(\boldsymbol{\lambda}^{\top} \boldsymbol{X}\right)}{\partial \lambda_{j}}\right|_{\boldsymbol{\lambda}=\mathbf{1}_{d}}, \quad \text { where } \quad \mathbf{1}_{d}=(1, \ldots, 1) \in \mathbb{R}^{d}
$$

provided that the partial derivative exists; see Tasche (2001) for more details on the differentiability argument. The Euler principle leads to the VaR contributions and ES contributions given by

$$
\left.\frac{\partial \varrho\left(\boldsymbol{\lambda}^{\top} \boldsymbol{X}\right)}{\partial \lambda_{j}}\right|_{\boldsymbol{\lambda}=\mathbf{1}_{d}}=\mathbb{E}\left[X_{j} \mid\left\{S=\operatorname{VaR}_{p}(S)\right\}\right] \quad \text { when } \varrho=\operatorname{VaR}_{p}
$$

and

$$
\left.\frac{\partial \varrho\left(\boldsymbol{\lambda}^{\top} \boldsymbol{X}\right)}{\partial \lambda_{j}}\right|_{\boldsymbol{\lambda}=\mathbf{1}_{d}}=\mathbb{E}\left[X_{j} \mid\left\{S \geq \operatorname{VaR}_{p}(S)\right\}\right], \quad \text { when } \varrho=\mathrm{ES}_{p},
$$

respectively.

We consider the case when the capital is an exogenously given constant $K \in \mathbb{R}$. Our proposed risk allocation introduced in Section 4 is based on the conditional distribution

$$
F_{\boldsymbol{X} \mid\{S=K\}}(\boldsymbol{x})=\mathbb{P}(\boldsymbol{X} \leq \boldsymbol{x} \mid\{S=K\}), \quad \boldsymbol{x} \in \mathbb{R}^{d} .
$$

The conditional distribution (4) is degenerate and its first $d^{\prime}=d-1$ components $\boldsymbol{X}^{\prime} \mid\{S=K\}=$ $\left(X_{1}, \ldots, X_{d^{\prime}}\right) \mid\{S=K\}$ determine the last one via $X_{d}\left|\{S=K\}=K-\left(X_{1}+\cdots+X_{d^{\prime}}\right)\right|\{S=K\}$. Therefore, it suffices to consider the $d^{\prime}$-dimensional marginal distribution $F_{\boldsymbol{X}^{\prime} \mid\{S=K\}}$. Note that throughout this paper, the '-notation is used to denote quantities related to this non-degenerate distribution in $d-1$ dimensions and should not be confused with matrix transposition for which we will use the ${ }^{\top}$-symbol. Assuming that $\boldsymbol{X}$ and $\left(\boldsymbol{X}^{\prime}, S\right)$ admit densities, $\boldsymbol{X}^{\prime} \mid\{S=K\}$ also has a density and is given by

$$
f_{\boldsymbol{X}^{\prime} \mid\{S=K\}}\left(\boldsymbol{x}^{\prime}\right)=\frac{f_{\left(\boldsymbol{X}^{\prime}, S\right)}\left(\boldsymbol{x}^{\prime}, K\right)}{f_{S}(K)}=\frac{f_{\boldsymbol{X}}\left(\boldsymbol{x}^{\prime}, K-\mathbf{1}_{d^{\prime}}^{\top} \boldsymbol{x}^{\prime}\right)}{f_{S}(K)}, \quad \boldsymbol{x}^{\prime} \in \mathbb{R}^{d^{\prime}},
$$

where the last equality follows from an affine transformation $\left(\boldsymbol{X}^{\prime}, S\right) \mapsto \boldsymbol{X}$ with unit Jacobian.

\subsection{A motivating example}

The distribution of $\boldsymbol{X} \mid\{S=K\}$ is a primary subject in this paper. In this section, we provide a motivating example for investigating this distribution from the viewpoint of risk alloation. 
(a)

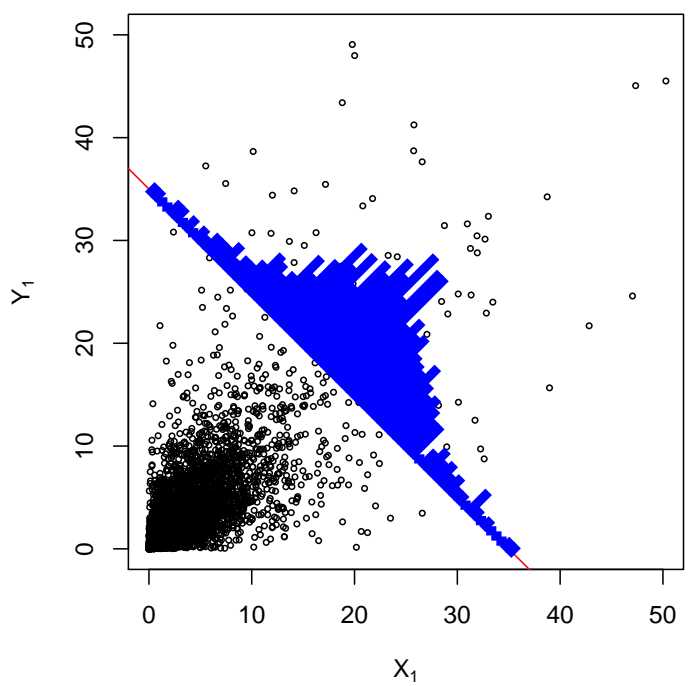

(b)

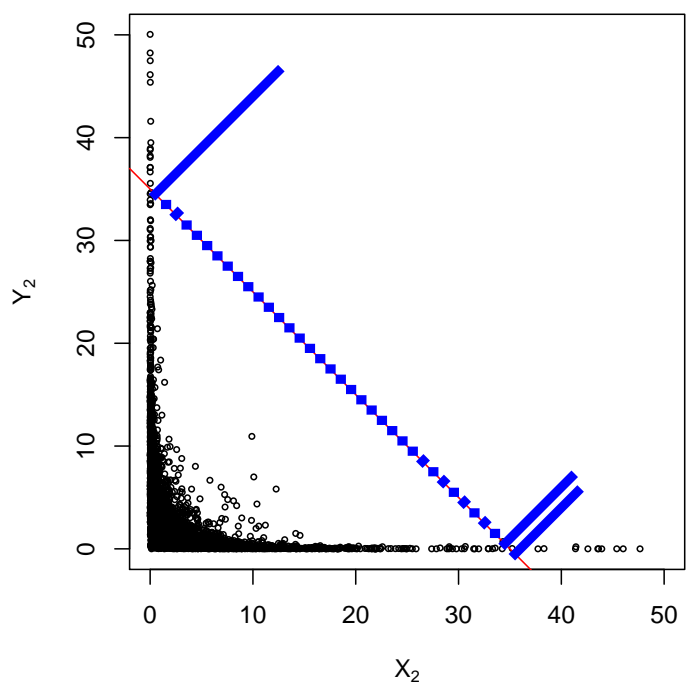

Figure 1: Scatter plots (black dots) of (a) $\left(X_{1}, Y_{1}\right)$ and (b) $\left(X_{2}, Y_{2}\right)$ such that all of $X_{1}, Y_{1}, X_{2}$ and $Y_{2}$ identically follow Pareto distributions with shape parameter 3 and scale parameter 5 , and $\left(X_{1}, Y_{1}\right)$ and $\left(X_{2}, Y_{2}\right)$ have Student $t$ copulas $C_{\nu, \rho_{1}}^{t}$ and $C_{\nu, \rho_{2}}^{t}$, respectively, where $\nu=5$ is the degrees of freedom, and $\rho_{1}=0.8$ and $\rho_{2}=-0.8$ are the correlation parameters. The red line indicates $x+y=K$ for $K=35$. Histograms (blue) of the conditional distributions of (a) $\left(X_{1}, Y_{1}\right)$ and (b) $\left(X_{2}, Y_{2}\right)$ on the (approximate) set of allocations $\left\{(x, y) \in \mathbb{R}^{2}: K-\delta<x+y<K+\delta\right\}, \delta=0.5$, are drawn on $\mathcal{K}_{d}(K)=\left\{(x, y) \in \mathbb{R}^{2}: x+y=K\right\}$.

\subsubsection{Soundness of risk allocations}

Consider two bivariate risks (a) $\left(X_{1}, Y_{1}\right)$ and (b) $\left(X_{2}, Y_{2}\right)$ such that all of $X_{1}, Y_{1}, X_{2}$ and $Y_{2}$ identically follow Pareto distributions with shape parameter 3 and scale parameter 5 , and $\left(X_{1}, Y_{1}\right)$ and $\left(X_{2}, Y_{2}\right)$ have Student $t$ copulas $C_{\nu, \rho_{1}}^{t}$ and $C_{\nu, \rho_{2}}^{t}$, respectively, where $\nu=5$ is the degrees of freedom parameter and $\rho_{1}=0.8$ and $\rho_{2}=-0.8$ are the correlation parameters. Suppose that the exogenously given total capital equals $K=35$. By exchangeability of the risk models (a) and (b), most allocation rules provide the homogeneous allocation $(K / 2, K / 2)=(17.5,17.5)$ in both cases (a) and (b). For instance, if $K$ is regarded as VaR or ES at some confidence levels and is allocated according to the Euler principle, then both VaR and ES contributions lead to homogeneous allocations. As we see in Figure 1, however, the conditional distributions of $\left(X_{1}, Y_{1}\right)$ and of $\left(X_{2}, Y_{2}\right)$ on the set of allocations $\mathcal{K}_{d}(K)$ differ substantially. Positive dependence among $X_{1}$ and $Y_{1}$ prevents the two random variables from moving in opposite directions under the constraint $X_{1}+Y_{1}=K$, which results in unimodality of the conditional distribution on $\mathcal{K}_{d}(K)$. On the other hand, negative dependence among $X_{2}$ and $Y_{2}$ allows them to move in opposite directions, which leads to bimodality of the conditional distribution. From the viewpoint of risk management, the homogeneous allocation $(K / 2, K / 2)$ seems to be a more sound capital allocation in Case (a) because it covers the most likely risky scenario. In Case (b), the two risky scenarios around the corners $(K, 0)$ and $(0, K)$ occur equally likely and the allocation $(K / 2, K / 2)$ can be understood as an average of these scenarios. However, the likelihood around $(K / 2, K / 2)$ is quite small and a single vector of the equal allocation $(K / 2, K / 2)$ obscures 
the two distinct risky scenarios. Moreover, either of $X_{2}$ or $Y_{2}$ is likely to suffer a large amount of loss if $(K / 2, K / 2)$ is reserved as capital. Consequently, the soundness of the allocated capital depends on the modality of the conditional loss distribution, and multiple risky scenarios can be hidden in a single vector of risk allocations

Remark 1 (Multimodality and variability). Models (a) and (b) may not be directly comparable since the total capital for Model (b) is typically smaller than that for Model (a). However, the variability of a risk under Model (b) is larger than under Model (a) in the sense that a wider variety of scenarios are likely to occur under Model (b) than under Model (a). Therefore, if the total capital $K$ does not incorporate the variability of a risk, an adjustment of $K$ may be required to take the multimodality of scenarios into account.

\subsubsection{Stress testing of risk allocations}

Inspecting modes of $\boldsymbol{X} \mid\{S=K\}$ can also be regarded as a stress test of risk allocations. Breuer et al. (2018) requires stress scenarios to be severe and plausible. We define the scenario set with a level of plausibility $t>0$ by $L_{t}(\boldsymbol{X})=\left\{\boldsymbol{x} \in \mathbb{R}^{d}: f_{\boldsymbol{X}}(\boldsymbol{x}) \geq t\right\}$ where $f_{\boldsymbol{X}}$ is the density function of $\boldsymbol{X}$ assuming that it exists. Among the scenario set $L_{t}(\boldsymbol{X})$, the set $L_{t}(\boldsymbol{X}) \cap \mathcal{K}_{d}(K)$ can be regarded as the set of the most severe scenarios the given total capital $K$ can cover. Using the convention $f_{\boldsymbol{X} \mid\{S=K\}}(\boldsymbol{x})=f_{\boldsymbol{X}}(\boldsymbol{x}) \mathbf{1}_{\left\{\mathbf{1}_{d}^{\top} \boldsymbol{x}=K\right\}} / f_{S}(K)$, $\boldsymbol{x} \in \mathbb{R}^{d}$, the set $L_{t}(\boldsymbol{X}) \cap \mathcal{K}_{d}(K)$ leads to the superlevel set of $\boldsymbol{X} \mid\{S=K\}$ with level $t / f_{S}(K)$ since

$$
\begin{aligned}
L_{t}(\boldsymbol{X}) \cap \mathcal{K}_{d}(K) & =\left\{\boldsymbol{x} \in \mathbb{R}^{d}: f_{\boldsymbol{X}}(\boldsymbol{x}) \mathbf{1}_{\left\{\mathbf{1}_{d}^{\top} \boldsymbol{x}=K\right\}} \geq t\right\} \\
& =\left\{\boldsymbol{x} \in \mathbb{R}^{d}: f_{\boldsymbol{X} \mid\{S=K\}}(\boldsymbol{x}) \geq t / f_{S}(K)\right\}=L_{t / f_{S}(K)}(\boldsymbol{X} \mid\{S=K\}) .
\end{aligned}
$$

Throughout the paper, the superlevel set of $\boldsymbol{X} \mid\{S=K\}$ is treated as a set of stress scenarios. In particular, the modes of $\boldsymbol{X} \mid\{S=K\}$ are the most severe and plausible scenarios that $K$ can cover since they attain the highest level of plausibility among the stress scenarios. Unimodality of $\boldsymbol{X} \mid\{S=K\}$ (see Definition 1 for its formal definition) implies that there exists one representative stress scenario the total capital $K$ can cover, and thus the mode can be a sound risk allocation covering the risky scenario most likely to occur. On the other hand, multimodality of $\boldsymbol{X} \mid\{S=K\}$ means that there are multiple distinct stress scenarios that are severe and plausible, and thus it may not be sufficient to only focus on a single scenario without identifying the other ones.

Remark 2 (Simulation of $\boldsymbol{X} \mid\{S=K\}$ with MCMC methods). Another motivation for investigating distributional properties of $\boldsymbol{X} \mid\{S=K\}$ is an efficient simulation of this conditional distribution. This is a challenging task in general since there is no general and tractable sampling method known for $\boldsymbol{X} \mid\{S=K\}$. Although samples from $\boldsymbol{X} \mid\{S=K\}$ can be generated by first simulating $\boldsymbol{X}$ and then extracting those satisfying the constraint $\{S=K\}$, the probability $\mathbb{P}(S=K)$ is zero, and thus such samples virtually never exist when $S$ admits a density. A potential remedy for this problem is to modify the conditioning set $\{S=K\}$ to $\{K-\delta<S<K+\delta\}$ for a small $\delta>0$ so that $P(K-\delta<S<K+\delta)>0$. However, this modification distorts the distribution of $\boldsymbol{X} \mid\{S=K\}$ and the resulting estimates of risk allocations

suffer from inevitable biases. To overcome this issue, Koike and Minami (2019) and Koike and Hofert (2020) proposed MCMC methods for exact simulation from $\boldsymbol{X} \mid\{S=K\}$. Although MCMC methods 
improve sample efficiency and the resulting estimates are unbiased, their performance highly depends on distributional properties of $\boldsymbol{X} \mid\{S=K\}$, in particular on its modality and tail behavior; see Appendix $\mathrm{F}$ for more details. From this viewpoint, investigating properties of $\boldsymbol{X} \mid\{S=K\}$ is of potential importance to construct efficient MCMC methods for simulating $\boldsymbol{X} \mid\{S=K\}$.

\section{Properties of the conditional distribution given a constant sum}

In Section 2.2, we showed that the conditional distribution of $\boldsymbol{X}$ given a constant sum $\{S=K\}$ plays an important role in risk allocations. With this motivation in mind, we study the support, modality, dependence and tail behavior of $\boldsymbol{X} \mid\{S=K\}$ in this section. As introduced in Section 2.1, we consider the $d^{\prime}$-dimensional random vector $\boldsymbol{X}^{\prime} \mid\{S=K\}$ for $d^{\prime}=d-1$ to avoid the degeneracy of $\boldsymbol{X} \mid\{S=K\}$.

\subsection{Support of $\boldsymbol{X} \mid\{S=K\}$}

We start with the support of $\boldsymbol{X}^{\prime} \mid\{S=K\}$. Assuming that $\boldsymbol{X}^{\prime} \mid\{S=K\}$ admits a density $f_{\boldsymbol{X}^{\prime} \mid\{S=K\}}$, Equation (5) implies that

$$
\operatorname{supp}\left(\boldsymbol{X}^{\prime} \mid\{S=K\}\right)=\left\{\boldsymbol{x}^{\prime} \in \mathbb{R}^{d^{\prime}}: f_{\boldsymbol{X}^{\prime} \mid\{S=K\}}\left(\boldsymbol{x}^{\prime}\right)>0\right\}=\left\{\boldsymbol{x}^{\prime} \in \mathbb{R}^{d^{\prime}}: f_{\boldsymbol{X}}\left(\boldsymbol{x}^{\prime}, K-\mathbf{1}_{d^{\prime}}^{\top} \boldsymbol{x}^{\prime}\right)>0\right\} .
$$

Therefore, if $X_{1}, \ldots, X_{d}$ are supported on $\mathbb{R}^{d}$, then $\operatorname{supp}\left(\boldsymbol{X}^{\prime} \mid\{S=K\}\right)=\mathbb{R}^{d^{\prime}}$. Another typical case is when $\operatorname{supp}(\boldsymbol{X})=\left(l_{1}, \infty\right) \times \cdots \times\left(l_{d}, \infty\right)$ for some $l_{1}, \ldots, l_{d}>-\infty$, which implies that $X_{1}, \ldots, X_{d}$ are bounded from below, that is, $X_{j} \geq l_{j} \mathbb{P}$-almost surely (a.s.), for $j=1, \ldots, d$. In this case, the support of $\boldsymbol{X}^{\prime} \mid\{S=K\}$ is given by

$$
\operatorname{supp}\left(\boldsymbol{X}^{\prime} \mid\{S=K\}\right)=\left\{\boldsymbol{x}^{\prime} \in \mathbb{R}^{d^{\prime}}: x_{1}>l_{1}, \ldots, x_{d^{\prime}}>l_{d^{\prime}}, \sum_{j=1}^{d^{\prime}} x_{j}^{\prime}<K-l_{d}\right\} .
$$

If $l_{1}=\cdots=l_{d}=0$, that is, when $\boldsymbol{X}$ models the nonnegative part of losses, then the closure of (6) is known as the $K$-simplex. Since the set in (6) is bounded, simulation of $\boldsymbol{X}^{\prime} \mid\{S=K\}$ can be more straightforward than in the former case when $\operatorname{supp}\left(\boldsymbol{X}^{\prime} \mid\{S=K\}\right)=\mathbb{R}^{d^{\prime}}$. For instance, an independent Metropolis-Hastings (MH) algorithm can be applied by first generating a sample $\boldsymbol{y}^{\prime}$ uniformly on the set in (6) and then replacing the current state $\boldsymbol{x}^{\prime}$ with the new state $\boldsymbol{y}^{\prime}$ with probability $\alpha\left(\boldsymbol{x}^{\prime}, \boldsymbol{y}^{\prime}\right)=f_{\boldsymbol{X}^{\prime} \mid\{S=K\}}\left(\boldsymbol{y}^{\prime}\right) / f_{\boldsymbol{X}^{\prime} \mid\{S=K\}}\left(\boldsymbol{x}^{\prime}\right)=f_{\boldsymbol{X}}\left(\boldsymbol{y}^{\prime}, K-\mathbf{1}_{d^{\prime}}^{\top} \boldsymbol{y}^{\prime}\right) / f_{\boldsymbol{X}}\left(\boldsymbol{x}^{\prime}, K-\mathbf{1}_{d^{\prime}}^{\top} \boldsymbol{x}^{\prime}\right)$.

\section{$3.2 X \mid\{S=K\}$ in the elliptical case}

Elliptical distributions are important exceptions for which the distribution of $\boldsymbol{X}^{\prime} \mid\{S=K\}$ can be derived explicitly. See Chapter 6 of McNeil et al. (2015) for applications of elliptical distributions to risk management. Throughout this work, the set of all $d \times d$ positive definite matrices is denoted as $\mathcal{M}_{+}^{d \times d}$. The characteristic function of a random vector $\boldsymbol{X}$ is given by $\phi_{\boldsymbol{X}}(\boldsymbol{t})=\mathbb{E}\left[\exp \left(i \boldsymbol{t}^{\top} \boldsymbol{X}\right)\right], \boldsymbol{t} \in \mathbb{R}^{d}$. If a function $\psi(t):[0, \infty) \rightarrow \mathbb{R}$ is such that $\psi\left(\boldsymbol{t}^{\top} \boldsymbol{t}\right)$ is a $d$-dimensional characteristic function, then $\psi$ is called a characteristic generator; see Fang (2018) for details. Let $\Psi_{d}$ denote the class of all characteristic generators. A $d$-dimensional random vector $\boldsymbol{X}$ is said to have an elliptical distribution, denoted by $\boldsymbol{X} \sim \mathcal{E}_{d}(\boldsymbol{\mu}, \Sigma, \psi)$, if 
its characteristic function can be expressed as

$$
\phi_{\boldsymbol{X}}(\boldsymbol{t})=\exp \left(i \boldsymbol{t}^{\top} \boldsymbol{\mu}\right) \psi\left(\frac{1}{2} \boldsymbol{t}^{\top} \Sigma \boldsymbol{t}\right)
$$

for a location vector $\boldsymbol{\mu} \in \mathbb{R}^{d}$, dispersion matrix $\Sigma \in \mathcal{M}_{+}^{d \times d}$ and a characteristic generator $\psi \in \Psi_{d}$. When an elliptical distribution $\boldsymbol{X} \sim \mathcal{E}_{d}(\boldsymbol{\mu}, \Sigma, \psi)$ admits a density function, it is of the form

$$
f_{\boldsymbol{X}}(\boldsymbol{x})=\frac{c_{d}}{\sqrt{|\Sigma|}} g\left(\frac{1}{2}(\boldsymbol{x}-\boldsymbol{\mu})^{\top} \Sigma^{-1}(\boldsymbol{x}-\boldsymbol{\mu}) ; d\right), \quad \boldsymbol{x} \in \mathbb{R}^{d}
$$

for some normalizing constant $c_{d}>0$ and a density generator $g(\cdot ; d)$ satisfying

$$
\int_{0}^{\infty} t^{d / 2-1} g(t ; d) \mathrm{d} t<\infty
$$

see Fang (2018). We omit the second argument and write $g(\cdot)=g(\cdot ; d)$ when it can be ignored.

In the following proposition we derive the distribution of $\boldsymbol{X}^{\prime} \mid\{S=K\}$ provided that $\boldsymbol{X} \sim \mathcal{E}_{d}(\boldsymbol{\mu}, \Sigma, \psi)$.

Proposition 1 (Ellipticality of $\left.\boldsymbol{X}^{\prime} \mid\{S=K\}\right)$. Suppose $\boldsymbol{X} \sim \mathcal{E}_{d}(\boldsymbol{\mu}, \Sigma, \psi)$. Then $\boldsymbol{X}^{\prime} \mid\{S=K\}$ follows an elliptical distribution $\mathcal{E}_{d^{\prime}}\left(\boldsymbol{\mu}_{K}, \Sigma_{K}, \psi_{K}\right)$ for some characteristic generator $\psi_{K} \in \Psi_{d^{\prime}}$,

$$
\boldsymbol{\mu}_{K}=\boldsymbol{\mu}^{\prime}+\frac{K-\mu_{S}}{\sigma_{S}^{2}}\left(\Sigma \mathbf{1}_{d}\right)^{\prime} \quad \text { and } \quad \Sigma_{K}=\Sigma^{\prime}-\frac{1}{\sigma_{S}^{2}}\left(\Sigma \mathbf{1}_{d}\right)^{\prime}\left(\Sigma \mathbf{1}_{d}\right)^{\prime \top},
$$

where $\boldsymbol{\mu}^{\prime}$ and $\left(\Sigma \mathbf{1}_{d}\right)^{\prime}$ are the first $d^{\prime}$-components of $\boldsymbol{\mu}$ and $\left(\Sigma \mathbf{1}_{d}\right)$, respectively, $\Sigma^{\prime}$ is the principal submatrix of $\Sigma$ deleting the dth row and column, $\mu_{S}=\mathbf{1}_{d}^{\top} \boldsymbol{\mu}$ and $\sigma_{S}^{2}=\mathbf{1}_{d}^{\top} \Sigma \mathbf{1}_{d}$. Furthermore, if $\boldsymbol{X}$ admits a density with density generator $g$, then $\boldsymbol{X}^{\prime} \mid\{S=K\}$ admits a density with density generator

$$
g_{K}(t)=g\left(t+\Delta_{K}\right) \quad \text { where } \quad \Delta_{K}=\frac{1}{2}\left(\frac{K-\mu_{S}}{\sigma_{S}}\right)^{2} .
$$

Note that the characteristic generator $\psi_{K}$ of $\boldsymbol{X}^{\prime} \mid\{S=K\}$ is in general different from that of $\boldsymbol{X}$; see the proof in Appendix A. By Proposition 1, ellipticality is preserved under conditioning $\{S=K\}$ and thus a change of the shape of the distribution as observed in Figure 1 (b) does not occur when $\boldsymbol{X}$ is elliptical. The capital $K$ is typically much larger than the mean of the total loss $\mu_{S}$ in practice. Therefore, by (8), the density generator $g_{K}$ is typically the tail part of the generator $g$. Moreover, the location vector $\boldsymbol{\mu}_{K}$ typically increases in proportion to the sum of covariances $\left(\Sigma \mathbf{1}_{d}\right)^{\prime}$. As a consequence, more (less) capital is assigned to losses which are positively (negatively) correlated with the other losses. On the other hand, the dispersion matrix $\Sigma_{K}$ decreases in proportion to the term $\left(\Sigma \mathbf{1}_{d}\right)^{\prime}\left(\Sigma \mathbf{1}_{d}\right)^{\prime \top}$ and the reduction depends on the variance of the sum.

Example 1 (Student $t$ distribution). A $d$-dimensional Student $t$ distribution $t_{\nu}(\boldsymbol{\mu}, \Sigma)$ is an elliptical distribution $\mathcal{E}_{d}(\boldsymbol{\mu}, \Sigma, \psi)$ with density generator

$$
g(t ; d)=\left(1+\frac{t}{\nu}\right)^{-\frac{d+\nu}{2}}, \quad t \geq 0,
$$

where $\nu \geq 1$ is the degrees of freedom parameter. It is known, for example, from Roth (2012) and Ding (2016) that the conditional distribution of the Student $t$ distribution is again Student $t$. We can check this 
closedness property with Proposition 1. By (8), the random variable $\boldsymbol{X}^{\prime} \mid\{S=K\}$ follows an elliptical distribution $\mathcal{E}_{d^{\prime}}\left(\boldsymbol{\mu}_{K}, \Sigma_{K}, g_{K}\right)$ with density generator (up to a constant) given by

$$
g_{K}(t)=\left(1+\frac{t}{\nu+\Delta_{K}}\right)^{-\frac{d+\nu}{2}}
$$

for which the corresponding distribution is known as the Pearson type VII distribution; see Schmidt (2002). In fact, this distribution reduces to a $d^{\prime}$-dimensional Student $t$ distribution since

$$
g_{K}(t)=\left(1+\frac{t}{\nu+\Delta_{K}}\right)^{-\frac{d+\nu}{2}} \propto\left(1+\frac{\nu+1}{\nu+\Delta_{K}} \frac{t}{\nu+1}\right)^{-\frac{d^{\prime}+\nu+1}{2}},
$$

and the multiplier $(\nu+1) /\left(\nu+\Delta_{K}\right)$ can be absorbed by redefining the dispersion matrix as $\tilde{\Sigma}_{K}=(\nu+$ $\left.\Delta_{K}\right) \Sigma_{K} /(\nu+1)$ for $\left(\nu+\Delta_{K}\right) /(\nu+1)>0$. Consequently, $\boldsymbol{X}^{\prime} \mid\{S=K\}$ follows $t_{\nu+1}\left(\boldsymbol{\mu}_{K}, \tilde{\Sigma}_{K}\right)$. Since the degrees of freedom of $\boldsymbol{X}^{\prime} \mid\{S=K\}$ increases by $1, \boldsymbol{X}^{\prime} \mid\{S=K\}$ has slightly lighter tails than $\boldsymbol{X}$.

\subsection{Unimodality of $\boldsymbol{X} \mid\{S=K\}$}

Next we study the modality of $\boldsymbol{X}^{\prime} \mid\{S=K\}$. Among various definitions of unimodality considered in the literature, we adopt those defined based on the superlevel set

$$
L_{t}(f)=\left\{\boldsymbol{x} \in \mathbb{R}^{d}: f(\boldsymbol{x}) \geq t\right\}, \quad t \in\left(0, \max \left\{f(\boldsymbol{x}): \boldsymbol{x} \in \mathbb{R}^{d}\right\}\right],
$$

where $f$ is a density on $\mathbb{R}^{d}$ which is assumed to be bounded for simplicity so that $\max \{f(\boldsymbol{x}): \boldsymbol{x} \in \mathbb{R}\}$ exists. By definition, $L_{t}(f)$ is a decreasing set, that is, $L_{t^{\prime}}(f) \subseteq L_{t}(f)$ for $0<t \leq t^{\prime}$. We also write $L_{t}(\boldsymbol{X})$ for $L_{t}(f)$ if $\boldsymbol{X}$ has density $f$. A set $A \subseteq \mathbb{R}^{d}$ is called star-shaped about $\boldsymbol{x}_{0} \in A$ if, for any $\boldsymbol{y} \in A$, the line segment from $\boldsymbol{x}_{0}$ to $\boldsymbol{y}$ is in $A$.

Definition 1 (Concepts of unimodality). For a bounded density function $f$ on $\mathbb{R}^{d}$, we call $M(f)=L_{t^{*}}(f)$ the mode set of $f$ for $t^{*}=\max \left\{f(\boldsymbol{x}): \boldsymbol{x} \in \mathbb{R}^{d}\right\}$. If $L_{t^{*}}(f)=\{\boldsymbol{m}\}$ then we call $\boldsymbol{m} \in \mathbb{R}^{d}$ the mode of $f$. Furthermore, $f$ is said to be weakly unimodal if $L_{t}(f)$ is connected, star unimodal about the center $\boldsymbol{x}_{0} \in \mathbb{R}^{d}$ if $L_{t}(f)$ is star-shaped about $\boldsymbol{x}_{0}$ and convex unimodal if $L_{t}(f)$ is convex, for all $0<t \leq t^{*}$. Finally, $f$ is said to be multimodal if $L_{t}(f)$ is not connected for some $0<t \leq t^{*}$.

From Definition 1, convex unimodality implies star unimodality and star unimodality implies weak unimodality. Other notions of unimodality, such as block unimodality, linear unimodality, monotone unimodality, $\alpha$-unimodality, orthounimodality and Khinchin's unimodality are not introduced in this paper due to their intractability for our purpose; see Dharmadhikari and Joag-Dev (1988) for a comprehensive discussion on unimodality. As mentioned in Section 2.2, $L_{t}(\boldsymbol{X})$ can be understood as a plausible scenario set with $t>0$ being the level of plausibility. In addition, $L_{t}(\boldsymbol{X} \mid\{S=K\})$ can be regarded as a set of severe and plausible stress scenarios the total capital $K$ is supposed to cover. From these interpretations, we believe that the notion of unimodality should describe tractability of these scenario sets, such as connectivity and convexity. The superlevel set $L_{t}(f)$ can also be important when $f$ is simulated with MCMC methods since the ratio of levels of $f$ is a primary quantity of interest for such methods. MCMC methods are required to be specifically designed when $L_{t}(f)$ is not connected since in this case a Markov chain needs to traverse distinct regions to simulate samples from the entire space. 
Note that uniqueness of the maximum of a density $f$, that is, the mode set of $f$ being a singleton $L_{t^{*}}(f)=\{\boldsymbol{m}\}$ for $\boldsymbol{m} \in \mathbb{R}^{d}$, is an important but different concept of unimodality from those in Definition 1 . In fact, uniqueness of the maximum may not be an appropriate concept of unimodality when the relationship between $\boldsymbol{X}$ and $\boldsymbol{X}^{\prime} \mid\{S=K\}$ is of interest. This is because the uniqueness of the maximum of $f_{\boldsymbol{X}^{\prime} \mid\{S=K\}}$ is equivalent to that of $f_{\boldsymbol{X}}$ on the restricted domain $\mathcal{K}_{d}(K)$ via $(5)$, and thus the uniqueness of the maximum of $f_{\boldsymbol{X}}$ on the entire support $\mathbb{R}^{d}$ does not provide any information on the shape of $f_{\boldsymbol{X}}$ on $\mathcal{K}_{d}(K)$ unless the mode of $f_{\boldsymbol{X}}$ on $\mathbb{R}^{d}$ is in $\mathcal{K}_{d}(K)$.

The following proposition reveals some relationships between unimodality of $\boldsymbol{X}$ and that of $\boldsymbol{X}^{\prime} \mid\{S=$ $K\}$.

Proposition 2 (Unimodality of $\boldsymbol{X}^{\prime} \mid\{S=K\}$ ).

1. Suppose $\boldsymbol{X} \sim \mathcal{E}_{d}(\boldsymbol{\mu}, \Sigma, \psi)$ admits a density with density generator $g$. If $g$ is decreasing on $\mathbb{R}_{+}$, then $f_{\boldsymbol{X}^{\prime} \mid\{S=K\}}$ is convex unimodal. Furthermore, if the equation $g(t)=\Delta_{K}$ of $t \in \mathbb{R}_{+}$has a unique solution $t_{K}^{*}$, then $f_{\boldsymbol{X}^{\prime} \mid\{S=K\}}$ has the mode $\boldsymbol{m}=\boldsymbol{\mu}_{K}$.

2. If $\boldsymbol{X}$ is convex unimodal, then $\boldsymbol{X}^{\prime} \mid\{S=K\}$ is convex unimodal.

Unlike convex unimodality, neither weak unimodality nor star unimodality of $\boldsymbol{X}$ implies any of the unimodality concepts of $\boldsymbol{X}^{\prime} \mid\{S=K\}$ introduced in Definition 1. To provide a counterexample, we introduce the following class of distributions.

Definition 2 (Homothetic density). A d-dimensional random vector $\boldsymbol{X}$ is said to have a homothetic density, denoted by $\boldsymbol{X} \sim \mathcal{H}(\boldsymbol{\mu}, D, r)$, with a location parameter $\boldsymbol{\mu} \in \mathbb{R}^{d}$, shape set $D \subseteq \mathbb{R}^{d}$ and a scaling function $r: \mathbb{R}_{+} \rightarrow \mathbb{R}_{+}$if $\boldsymbol{X}-\boldsymbol{\mu}$ admits a density $f_{D}$ satisfying

$$
L_{t}\left(f_{D}\right)=r(t) D=\{s \boldsymbol{x}: 0 \leq s \leq r(t), \boldsymbol{x} \in D\}
$$

for some continuous and decreasing function $r$ and a bounded and star-shaped set $D \in \mathbb{R}^{d}$ around $\mathbf{0}$ such that

$$
\int_{0}^{\infty} \operatorname{Leb}_{d}(r(t) D) \mathrm{d} t=1
$$

where $\mathrm{Leb}_{d}$ denotes the Lebesgue measure on $\mathbb{R}^{d}$.

Note that Condition (10) is required to ensure that $\int_{\mathbb{R}^{d}} f_{D}(\boldsymbol{x}) \mathrm{d} \boldsymbol{x}=1$. To see this, we have

$$
\begin{aligned}
\int_{\mathbb{R}^{d}} f_{D}(\boldsymbol{x}) \mathrm{d} \boldsymbol{x} & =\int_{\mathbb{R}^{d}} \int_{0}^{f_{D}(\boldsymbol{x})} \mathrm{d} t \mathrm{~d} \boldsymbol{x}=\int_{\mathbb{R}^{d}} \int_{0}^{\infty} \mathbf{1}_{\left\{\boldsymbol{x} \in L_{t}\left(f_{D}\right)\right\}} \mathrm{d} t \mathrm{~d} \boldsymbol{x} \\
& =\int_{0}^{\infty} \operatorname{Leb}_{d}\left(L_{t}\left(f_{D}\right)\right) \mathrm{d} t=\int_{0}^{\infty} \operatorname{Leb}_{d}(r(t) D) \mathrm{d} t=1 .
\end{aligned}
$$

Homothetic distributions arise from $l_{p}$-spherical distributions (Osiewalski, 1993) where the superlevel sets are determined as balls in the $l_{p}$-norm, and also arise from a further generalized class of distributions called the $v$-spherical distributions (Fernandez et al., 1995). Examples of homothetic distributions include skew-normal distributions and rotund-exponential distributions; see Balkema and Nolde (2010). It is straightforward to check that $\boldsymbol{X} \sim \mathcal{H}\left(\mathbf{0}_{d}, D, r\right)$ is star unimodal about $\boldsymbol{x}_{0} \in \mathbb{R}^{d}$ if $D$ is star-shaped about $\boldsymbol{x}_{0}$, and convex unimodal if $D$ is convex. 
Suppose $\boldsymbol{X} \sim \mathcal{H}\left(\mathbf{0}_{d}, D, r\right)$ for a convex set $D$. Then $\boldsymbol{X}$ is convex unimodal and so is $\boldsymbol{X}^{\prime} \mid\{S=K\}$ by Proposition 2 Part 2. For this homothetic distribution, the superlevel set of $\boldsymbol{X}^{\prime} \mid\{S=K\}$ embedded in $\mathbb{R}^{d}$ has the following representation

$$
\begin{aligned}
\left\{\boldsymbol{x} \in \mathbb{R}^{d}\right. & \left.: \boldsymbol{x}^{\prime} \in L_{t}\left(\boldsymbol{X}^{\prime} \mid S=K\right), x_{d}=K-\mathbf{1}_{d^{\prime}}^{\top} \boldsymbol{x}^{\prime}\right\} \\
& =\left\{\boldsymbol{x} \in \mathbb{R}^{d}: f_{\boldsymbol{X}^{\prime} \mid\{S=K\}}\left(\boldsymbol{x}^{\prime}\right) \geq t, x_{d}=K-\mathbf{1}_{d^{\prime}}^{\top} \boldsymbol{x}^{\prime}\right\} \\
& =\left\{\boldsymbol{x} \in \mathbb{R}^{d}: f(\boldsymbol{x}) \geq t f_{S}(K)\right\} \cap \mathcal{K}_{d}(K)=L_{t f_{S}(K)}(f) \cap \mathcal{K}_{d}(K) \\
& =r\left(t f_{S}(K)\right) D \cap \mathcal{K}_{d}(K)=\left\{s \boldsymbol{x}: \boldsymbol{x} \in D, 0 \leq s \leq r\left(t f_{S}(K)\right)\right\} \cap \mathcal{K}_{d}(K) \\
& =\left\{\frac{K}{\mathbf{1}_{d}^{\top} \boldsymbol{x}} \boldsymbol{x}: \boldsymbol{x} \in D, 0 \leq \frac{K}{\mathbf{1}_{d}^{\top} \boldsymbol{x}} \leq r\left(t f_{S}(K)\right)\right\} \\
& =\left\{\frac{K}{\mathbf{1}_{d}^{\top} \boldsymbol{x}} \boldsymbol{x}: \boldsymbol{x} \in \bigcup_{k \geq K / r\left(t f_{S}(K)\right)} D \cap \mathcal{K}_{d}(k)\right\},
\end{aligned}
$$

that is, the superlevel set $L_{t}\left(\boldsymbol{X}^{\prime} \mid\{S=K\}\right)$ embedded in $\mathbb{R}^{d}$ is a collection of the projected points of $\boldsymbol{x} \in D$ intersected with the upper half space $\left\{\boldsymbol{x} \in \mathbb{R}^{d}: \mathbf{1}_{d}^{\top} \boldsymbol{x} \geq K / r\left(t f_{S}(K)\right)\right\}$ onto $\mathcal{K}_{d}(K)$.

The following example shows that neither weak unimodality nor star unimodality of $\boldsymbol{X}$ implies any of the unimodality concepts introduced in Definition 1 for $\boldsymbol{X}^{\prime} \mid\{S=K\}$.

Example 2. Consider $\boldsymbol{X} \in \mathcal{H}\left(\mathbf{0}_{2}, D, r\right)$ where $D=([-2,2] \times[-1,1]) \cup([-1,1] \times[-2,2])$ and $r(t)=$ $\frac{1}{2 \sqrt{3}} \exp (-t / 2)$. $D$ is star-shaped (and thus connected) around $(0,0)$ and $r$ is a decreasing function. Furthermore, the pair of $(D, r)$ satisfies Condition (10) since

$$
\int_{0}^{\infty} \operatorname{Leb}_{2}(r(t) D) \mathrm{d} t=\operatorname{Leb}_{2}(D) \int_{0}^{\infty} r^{2}(t) \mathrm{d} t=12 \int_{0}^{\infty} \frac{1}{12} \exp (-t) \mathrm{d} t=1 .
$$

Suppose that the total capital is given by $K=1 / 3$. For $t=-2 \log (\sqrt{3} / 3) \approx 1.098$, we have $r(t)=1 / 6$ and thus $L_{t}\left(f_{D}\right)=D / 6=([-1 / 3,1 / 3] \times[-1 / 6,1 / 6]) \cup([-1 / 6,1 / 6] \times[-1 / 3,1 / 3])$. Therefore, $L_{t}\left(\boldsymbol{X}^{\prime} \mid\{S=\right.$ $K\})=[0,1 / 6] \cup[1 / 3,1 / 2]$, which is neither star-shaped nor even connected.

Next we study marginal properties of unimodality. In general, even if $\boldsymbol{X}$ is convex unimodal, it does not imply any unimodality for its marginal distributions; see Balkema and Nolde (2010, Example A.3.) for a counterexample. The following example shows that marginal unimodality also does not imply joint unimodality.

Example 3 (Marginal unimodality does not imply joint unimodality). Consider the following bivariate density

$$
f(u, v)=\frac{9}{4} \mathbf{1}_{\left\{(u, v) \in \bigcup_{i=1}^{3}[(i-1) / 3, i / 3]^{2}\right\}}+\frac{9}{4} \mathbf{1}_{\left\{(u, v) \in[1 / 3,2 / 3]^{2}\right\}}, \quad(u, v) \in[0,1],
$$

which has the convex unimodal marginal densities

$$
f_{1}(u)=f_{2}(u)=\frac{3}{4} \mathbf{1}_{\{u \in[0,1]\}}+\frac{3}{4} \mathbf{1}_{\{u \in[1 / 3,2 / 3]\}}, \quad u \in[0,1] .
$$

However, $L_{9 / 4}(f)=[0,1 / 3]^{2} \cup[1 / 3,2 / 3]^{2} \cup[2 / 3,1]^{2}$ is neither convex nor star-shaped.

Joint unimodality implies marginal unimodality for certain classes of distributions. As is shown in Balkema and Nolde (2010), $l_{p}$-spherical distributions form a subclass of homothetic densities for which unimodality is preserved under marginalization. This property also holds for the class of $s$-concave densities, which is also closed under the operation $\boldsymbol{X} \mapsto \boldsymbol{X}^{\prime} \mid\{S=K\}$; see Appendix B for details. 


\subsection{Dependence of $\boldsymbol{X} \mid\{S=K\}$ and its modality}

The dependence structure of $\boldsymbol{X}^{\prime} \mid\{S=K\}$ is typically described in terms of the dependence among $X_{j}$ and $S$ for $j=1, \ldots, d^{\prime}$. For instance, when $\boldsymbol{X} \sim \mathcal{E}_{d}(\boldsymbol{\mu}, \Sigma, \psi)$, Proposition 1 yields

$$
\begin{aligned}
\operatorname{Cov}\left[X_{i}, X_{j} \mid\{S=K\}\right] & =\left(\Sigma_{K}\right)_{i, j}=\operatorname{Cov}\left[X_{i}, X_{j}\right]-\frac{1}{\sigma_{S}^{2}}\left(\Sigma \mathbf{1}_{d}\right)_{i}\left(\Sigma \mathbf{1}_{d}\right)_{j} \\
& =\operatorname{Cov}\left[X_{i}, X_{j}\right]-\frac{1}{\sigma_{S}^{2}} \operatorname{Cov}\left[X_{i}, S\right] \operatorname{Cov}\left[X_{j}, S\right]=\sigma_{i} \sigma_{j}\left(\rho_{X_{i}, X_{j}}-\rho_{X_{i}, S} \rho_{X_{j}, S}\right),
\end{aligned}
$$

where $\sigma_{j}^{2}=\operatorname{Var}\left(X_{j}\right)$ and $\rho_{X_{i}, X_{j}}$ is the correlation coefficient of $\left(X_{i}, X_{j}\right)$. Beyond the elliptical case, various dependence concepts, in particular the total positivity and its related order of $\boldsymbol{X}^{\prime} \mid\{S=K\}$, are investigated in Appendix C.

In this section, we investigate the modality of $\boldsymbol{X} \mid\{S=K\}$ under extremal dependence of $\boldsymbol{X}$. The following example shows that $\boldsymbol{X} \mid\{S=K\}$ is degenerate and thus unimodal when $\boldsymbol{X}$ is comonotone.

Example $4\left(\boldsymbol{X}^{\prime} \mid\{S=K\}\right.$ under comonotonicity). Suppose $\boldsymbol{X}$ is a comonotone random vector with continuous and strictly increasing margins $F_{1}, \ldots, F_{d}$, that is, $\boldsymbol{X} \stackrel{\mathrm{d}}{=}\left(F_{1}^{-1}(U), \ldots, F_{d}^{-1}(U)\right)$ for some $U \sim$ $\mathrm{U}(0,1)$. Under continuity and strict increasingness of $F_{1}, \ldots, F_{d}$, their quantile functions $F_{j}^{-1}, j=1, \ldots, d$, are continuous and strictly increasing. Therefore, the equation $\sum_{j=1}^{d} F_{j}^{-1}(u)=K$ of $u \in[0,1]$ has a unique solution $u^{*}$. Moreover, it holds that

$$
\boldsymbol{X} \mid\{S=K\}=\left(F_{1}^{-1}\left(u^{*}\right), \ldots, F_{d}^{-1}\left(u^{*}\right)\right) \quad \mathbb{P} \text {-a.s. }
$$

since

$$
\mathbb{P}\left(\bigcup_{j=1}^{d}\left\{X_{j} \neq F_{j}^{-1}\left(u^{*}\right)\right\} \mid\{S=K\}\right)=\mathbb{P}\left(\bigcup_{j=1}^{d}\left\{F_{j}^{-1}(U) \neq F_{j}^{-1}\left(u^{*}\right)\right\} \mid\left\{\sum_{j=1}^{d} F_{j}^{-1}(U)=K\right\}\right)=0 .
$$

This example can be understood as an extreme case where positive dependence (comonotonicity) implies unimodality of $\boldsymbol{X} \mid\{S=K\}$, which takes on one point $\left(F_{1}^{-1}\left(u^{*}\right), \ldots, F_{d}^{-1}\left(u^{*}\right)\right)$ with probability 1 .

When $\boldsymbol{X}$ has negative dependence, a wider variety of distributions, possibly multimodal ones, arise as $\boldsymbol{X} \mid\{S=K\}$ compared with the positive dependent case. We demonstrate this phenomenon in the following example.

Example $5(\boldsymbol{X} \mid\{S=K\}$ under extreme negative dependence). Let $K>0$ and $X \sim F$ for a continuous distribution function $F$ supported on $[0, \infty)$ such that $X \mid\{X \leq K\}$ is radially symmetric about $K / 2$ in the sense that $(X-K / 2)|\{X \leq K\} \stackrel{\mathrm{d}}{=}(K / 2-X)|\{X \leq K\}$. For $U \sim \mathrm{U}(0,1)$ define $\left(X_{1}, X_{2}\right)$ by

$$
\begin{aligned}
& X_{1}=F^{-1}(U) \mathbf{1}_{\{U \leq F(K)\}}+F^{-1}(U) \mathbf{1}_{\{U>F(K)\}}=F^{-1}(U), \\
& X_{2}=\left(K-F^{-1}(U)\right) \mathbf{1}_{\{U \leq F(K)\}}+F^{-1}(U) \mathbf{1}_{\{U>F(K)\}} .
\end{aligned}
$$

Then $\mathbb{P}\left(X_{1} \leq x\right)=\mathbb{P}\left(F^{-1}(U) \leq x\right)=\mathbb{P}(U \leq F(x))=F(x)$ for all $x \geq 0$. Since the conditional radial symmetry of $F$ implies that $\mathbb{P}\left(K-F^{-1}(U) \leq x, U \leq F(K)\right)=\mathbb{P}\left(F^{-1}(U) \leq x, U \leq F(K)\right)$, it holds that

$$
\begin{aligned}
\mathbb{P}\left(X_{2} \leq x\right) & =\mathbb{P}\left(X_{2} \leq x, U \leq F(K)\right)+\mathbb{P}\left(X_{2} \leq x, U>F(K)\right) \\
& =\mathbb{P}\left(K-F^{-1}(U) \leq x, U \leq F(K)\right)+\mathbb{P}\left(F^{-1}(U) \leq x, U>F(K)\right) \\
& =\mathbb{P}\left(F^{-1}(U) \leq x, U \leq F(K)\right)+\mathbb{P}\left(F^{-1}(U) \leq x, U>F(K)\right) \\
& =\mathbb{P}\left(F^{-1}(U) \leq x\right)=F(x), \quad x \geq 0 .
\end{aligned}
$$


Therefore, $X_{1} \sim F$ and $X_{2} \sim F$. The body $\left\{X_{1} \leq K\right\}$ of $X_{1}$ and the tail $\left\{X_{2}>K\right\}$ of $X_{2}$ are mutually exclusive in the sense that $\mathbb{P}\left(X_{1} \leq K, X_{2}>K\right)=0$. Similarly $\mathbb{P}\left(X_{1}>K, X_{2} \leq K\right)=0$. In the tail, $X_{1}$ and $X_{2}$ are comonotone in the sense that $\left(X_{1}, X_{2}\right)=\left(F^{-1}(U), F^{-1}(U)\right)$ on $\{U>F(K)\}$. In the body, $X_{1}$ and $X_{2}$ are countermonotone in the sense that $\left(X_{1}, X_{2}\right)=\left(F^{-1}(U), K-F^{-1}(U)\right)$ on $\{U \leq F(K)\}$. Since $X_{1}+X_{2}=F^{-1}(U)+K-F^{-1}(U)=K$ on $\{U \leq F(K)\}$ and $X_{1}+X_{2}=2 F^{-1}(U)>2 K>K$ on $\{U>F(K)\}$, we have that

$$
\left\{X_{1}+X_{2}=K\right\}=\left\{X_{1}+X_{2}=K, U \leq F(K)\right\} \cup\left\{X_{1}+X_{2}=K, U>F(K)\right\}=\{U \leq F(K)\},
$$

and thus that

$$
\left(X_{1}, X_{2}\right)\left|\left\{X_{1}+X_{2}=K\right\}=\left(X_{1}, X_{2}\right)\right|\{U \leq F(K)\}=\left(F^{-1}(U), K-F^{-1}(U)\right) \mid\{U \leq F(K)\} .
$$

Consequently, $\left(X_{1}, X_{2}\right) \mid\{S=K\}$ has the homogeneous marginal distribution $F_{X \mid\{X \leq K\}}$ and a countermonotone copula $W$. Therefore, multimodality of $\boldsymbol{X} \mid\{S=K\}$ appears when, for example, $X \sim F$ has a bimodal distribution on the body $\{X \leq K\}$; see Remark 3 for a more concrete example.

Remark 3 (Extension to $d \geq 3$ with complete mixability). Example 5 for constructing $\left(X_{1}, X_{2}\right)$ based on countermonotonicity can be extended to the multivariate case $d \geq 3$. Let $K>0$ and $X \sim F$ for a continuous distribution function $F$ supported on $[0, \infty)$ such that the conditional distribution $F_{X \mid\{X \leq K\}}$ is $d$-completely mixable with center $K$ for $d \geq 3$, that is, there exists a $d$-dimensional random vector $\boldsymbol{Y}=\left(Y_{1}, \ldots, Y_{d}\right)$ called the $d$-complete mix such that $Y_{j} \sim F_{X \mid\{X \leq K\}}, j=1, \ldots, d$, and $Y_{1}+\cdots+Y_{d}=K$ a.s. Such a random vector exists, for example, when $F_{X \mid\{X \leq K\}}$ admits a decreasing density with $\mathbb{E}\left[Y_{1}\right]=K / d$; see Wang and Wang (2011, Corollary 2.9.). Define $\boldsymbol{X}=\left(X_{1}, \ldots, X_{d}\right)$ by $X_{j}=Y_{j} \mathbf{1}_{\{U \leq F(K)\}}+Z_{j} \mathbf{1}_{\{U>F(K)\}}$ for $\boldsymbol{Y}=\left(Y_{1}, \ldots, Y_{d}\right)$ being the $d$-complete mix of $F_{X \mid\{X \leq K\}}, U \sim \mathrm{U}(0,1), Z_{j} \sim F_{X \mid\{X>K\}}, j=1, \ldots, d$ and $\boldsymbol{Y}, U$ and $Z_{1}, \ldots, Z_{d}$ are independent of each other. Then one can check that $X_{j} \sim F$. Moreover, $\left\{X_{1}+\cdots+X_{d}=K\right\}=\{U \leq F(K)\}$ since

$$
S=X_{1}+\cdots+X_{d}=K \mathbf{1}_{\{U \leq F(K)\}}+\left(Z_{1}+\cdots+Z_{d}\right) \mathbf{1}_{\{U>F(K)\}},
$$

and $Z_{1}+\cdots+Z_{d}>d K>K$ a.s. Consequently, $\boldsymbol{X}|\{S=K\}=\boldsymbol{X}|\{U \leq F(K)\}=\boldsymbol{Y}$ a.s. and thus $\boldsymbol{X} \mid\{S=K\}$ is the $d$-complete mix of $X \mid\{X \leq K\}$. To construct a multimodal $\boldsymbol{X} \mid\{S=K\}$ one can choose $\boldsymbol{Y}$ as an equally weighted mixture of three Dirichlet distributions $\operatorname{Dir}(\alpha, \alpha, \beta), \operatorname{Dir}(\alpha, \beta, \alpha)$ and $\operatorname{Dir}(\beta, \alpha, \alpha)$ for $0<\alpha<\beta$. This mixture is a 3 -complete mix since it has homogeneous marginal distributions and a constant sum. Moreover, $\boldsymbol{Y}$ has non-connected superlevel sets when, for example, $\alpha=2$ and $\beta=10$, and thus $\boldsymbol{X}^{\prime} \mid\{S=K\}$ is multimodal.

\subsection{Tail behavior of $\boldsymbol{X} \mid\{S=K\}$}

In this section we study the tail behavior of $\boldsymbol{X}^{\prime} \mid\{S=K\}$ through its density. Since boundedness of $\boldsymbol{X}$ from below leads to a bounded support of $\boldsymbol{X}^{\prime} \mid\{S=K\}$ as shown in Section 3.1, we focus on the case when $\boldsymbol{X}$ is supported on $\mathbb{R}^{d}$. In this case, the support of $\boldsymbol{X}^{\prime} \mid\{S=K\}$ is $\mathbb{R}^{d^{\prime}}$ and thus there are $2^{d^{\prime}}$ orthants to be considered. Hereafter we consider tail behavior only in the first orthant $\left\{\boldsymbol{x}^{\prime} \in \mathbb{R}^{d^{\prime}}: x_{1}, \ldots, x_{d^{\prime}}>0\right\}$ since tails on the other orthants can be discussed similarly. We study the following limiting behaviors of the ratio of densities. 
Definition 3 (Multivariate regular and rapid variations of a density). Let $\boldsymbol{X}$ be a d-dimensional random vector $\boldsymbol{X}$ with a density $f_{\boldsymbol{X}}$.

1. $\boldsymbol{X}$ is called multivariate regularly varying with limit function $\lambda: \mathbb{R}^{2 d} \rightarrow \mathbb{R}_{+}$(at $\infty$ and on the first orthant), denoted by $\operatorname{MRV}(\lambda)$, if

$$
\lim _{t \rightarrow \infty} \frac{f_{\boldsymbol{X}}(t \boldsymbol{y})}{f_{\boldsymbol{X}}(t \boldsymbol{x})}=\lambda(\boldsymbol{x}, \boldsymbol{y})>0 \quad \text { for any } \quad \boldsymbol{x}, \boldsymbol{y} \in \mathbb{R}_{+}^{d},
$$

provided the limit function $\lambda$ exists.

2. $\boldsymbol{X}$ is called multivariate rapidly varying (at $\infty$ and on the first orthant), denoted by $M R V(\infty)$ if,

$$
\lim _{t \rightarrow \infty} \frac{f_{\boldsymbol{X}}(s t \boldsymbol{x})}{f_{\boldsymbol{X}}(t \boldsymbol{x})}=\left\{\begin{array}{ll}
0, & s>1, \\
\infty, & 0<s<1,
\end{array} \quad \text { for any } \quad s>0 \quad \text { and } \boldsymbol{x} \in \mathbb{R}_{+}^{d} .\right.
$$

Note that we adopt the definitions of regular and rapid variations of densities for their potential application to MCMC methods where the ratio of target densities $f_{\boldsymbol{X}^{\prime} \mid\{S=K\}}\left(\boldsymbol{y}^{\prime}\right) / f_{\boldsymbol{X}^{\prime} \mid\{S=K\}}\left(\boldsymbol{x}^{\prime}\right)$ at any two points $\boldsymbol{x}^{\prime}, \boldsymbol{y}^{\prime} \in \mathbb{R}^{d^{\prime}}$ is of interest; see Appendix F. Taking $\boldsymbol{x}=\mathbf{1}_{d}$ in (11) leads to the standard definition of regular variation introduced, for example, in Resnick (2007). Regular variation is typically described in terms of probability measures or their survival functions, and these concepts of variations are connected to regular variation of densities through Resnick (2007, Theorem 6.4.).

The following proposition states that one can find a limit function for $\boldsymbol{X}^{\prime} \mid\{S=K\}$ based on that of $\boldsymbol{X}$ through the auxiliary random vector $\tilde{\boldsymbol{X}}=\left(\boldsymbol{X}^{\prime}, K-X_{d}\right)$.

Proposition 3 (Multivariate regular and rapid variations of $\boldsymbol{X}^{\prime} \mid\{S=K\}$ ).

1. Assume that $\tilde{\boldsymbol{X}}=\left(\boldsymbol{X}^{\prime}, K-X_{d}\right)$ is $M R V(\tilde{\lambda})$. Then $\boldsymbol{X}^{\prime} \mid\{S=K\}$ is $M R V\left(\lambda^{\prime}\right)$ with limit function

$$
\lambda^{\prime}\left(\boldsymbol{x}^{\prime}, \boldsymbol{y}^{\prime}\right)=\tilde{\lambda}\left(\left(\boldsymbol{x}^{\prime}, \mathbf{1}_{d^{\prime}}^{\top} \boldsymbol{x}^{\prime}\right),\left(\boldsymbol{y}^{\prime}, \mathbf{1}_{d^{\prime}}^{\top} \boldsymbol{y}^{\prime}\right)\right), \quad \boldsymbol{x}^{\prime}, \boldsymbol{y}^{\prime} \in \mathbb{R}_{+}^{d^{\prime}} .
$$

2. If $\tilde{\boldsymbol{X}}$ is $M R V(\infty)$, then $\boldsymbol{X}^{\prime} \mid\{S=K\}$ is $M R V(\infty)$.

The sufficient conditions in Proposition 3 are straightforward to check since $\tilde{\boldsymbol{X}}$ does not depend on the sum $S$, and the joint distribution of $\tilde{\boldsymbol{X}}$ can be specified through its marginal distributions and copula. The margins of $\tilde{\boldsymbol{X}}$ are $\tilde{F}_{j}=F_{j}, j=1, \ldots, d^{\prime}$, and $\tilde{F}_{d}\left(x_{d}\right)=\bar{F}_{d}\left(K-x_{d}\right)$, and the copula $\tilde{C}$ of $\tilde{\boldsymbol{X}}$ is the distribution function of $\left(U_{1}, \ldots, U_{d^{\prime}}, 1-U_{d}\right)$ where $\boldsymbol{U} \sim C$ is the copula of $\boldsymbol{X}$. This enables one to find a limit function for $\tilde{\boldsymbol{X}}$; see, for example, Li (2013) and Joe and Li (2019).

The following proposition shows that the limit function is determined by the density generator $g$ in the elliptical case.

Proposition 4 (Multivariate regular and rapid variations for elliptical distribution). Assume $\boldsymbol{X} \sim \mathcal{E}_{d}(\boldsymbol{\mu}, \Sigma, \psi)$ admits a density with density generator $g$ continuous on $\mathbb{R}_{+}$.

1. If $g$ is regularly varying in the sense that

$$
\lim _{t \rightarrow \infty} \frac{g(t u)}{g(t s)}=\lambda_{g}(s, u), \quad s, u>0,
$$

then $\boldsymbol{X}^{\prime} \mid\{S=K\}$ is $\operatorname{MRV}\left(\lambda_{K}\right)$ with

$$
\lambda_{K}\left(\boldsymbol{x}^{\prime}, \boldsymbol{y}^{\prime}\right)=\lambda_{g}\left(\boldsymbol{x}^{\prime \top} \Sigma_{K}^{-1} \boldsymbol{x}^{\prime}, \boldsymbol{y}^{\prime \top} \Sigma_{K}^{-1} \boldsymbol{y}^{\prime}\right), \quad \boldsymbol{x}^{\prime}, \boldsymbol{y}^{\prime} \in \mathbb{R}^{d^{\prime}} .
$$


2. If $g$ is rapidly varying in the sense that

$$
\lim _{t \rightarrow \infty} \frac{g(s t)}{g(t)}= \begin{cases}0, & s>1 \\ \infty, & 0<s<1\end{cases}
$$

then $\boldsymbol{X}^{\prime} \mid\{S=K\}$ is $M R V(\infty)$.

Example 6 (Normal and Student $t$ distributions). The multivariate Normal distribution has a rapidly varying density generator $g(t)=\exp (-t)$, and thus its corresponding conditional distribution $\boldsymbol{X}^{\prime} \mid\{S=K\}$ is also rapidly varying by Proposition 4 Part 2. Next, suppose $\boldsymbol{X}$ follows a $d$-dimensional Student $t$ distribution with degrees of freedom $\nu \geq 1$. Its density generator (9) is regularly varying with limit function

$$
\lim _{t \rightarrow \infty} \frac{g(t u)}{g(t s)}=\left(\frac{u}{s}\right)^{-\frac{\nu+d}{2}}, \quad u, s>0 .
$$

Consequently, by Proposition 4 Part $1, \boldsymbol{X}^{\prime} \mid\{S=K\}$ is regularly varying with limit function

$$
\lim _{t \rightarrow \infty} \frac{f_{\boldsymbol{X}^{\prime} \mid\{S=K\}}\left(t \boldsymbol{y}^{\prime}\right)}{f_{\boldsymbol{X}^{\prime} \mid\{S=K\}}\left(t \boldsymbol{x}^{\prime}\right)}=\left(\frac{\left\|\Sigma_{K}^{-\frac{1}{2}} \boldsymbol{y}^{\prime}\right\|}{\left\|\Sigma_{K}^{-\frac{1}{2}} \boldsymbol{x}^{\prime}\right\|}\right)^{-(\nu+d)}, \quad \boldsymbol{x}^{\prime}, \boldsymbol{y}^{\prime} \in \mathbb{R}_{+}^{d^{\prime}},
$$

where $\|\cdot\|$ is an Euclidean norm on $\mathbb{R}^{d^{\prime}}$.

\section{Maximum likelihood allocation and multimodality adjustment}

In this section we investigate how the modality of $\boldsymbol{X} \mid\{S=K\}$ can be incorporated in risk management. Under unimodality, the mode of $\boldsymbol{X} \mid\{S=K\}$ is regarded as the most likely stress scenario covered by the given total capital $K$. This mode is defined to be a maximum likelihood allocation (MLA) in Section 4.1.1, and its properties are studied in Section 4.1.2. Under multimodality of $\boldsymbol{X} \mid\{S=K\}$, a single vector of allocations may obscure multiple risky scenarios as seen in Section 2.2. To overcome this issue, we introduce the so-called multimodality adjustment in Section 4.2 to utilize the knowledge of multimodality of $\boldsymbol{X} \mid\{S=K\}$ and to increase the soundness of risk allocations.

\subsection{Maximum likelihood allocation}

\subsubsection{Definition and assumptions on MLA}

We denote by $\mathcal{U}_{d}(K)$ the set of all $d$-dimensional random vectors $\boldsymbol{X}$ such that $\boldsymbol{X}$ and $\left(\boldsymbol{X}^{\prime}, S\right)$ admit density functions, and that the function $\boldsymbol{x} \mapsto f_{\boldsymbol{X}}(\boldsymbol{x}) \mathbf{1}_{\left\{\boldsymbol{x} \in \mathcal{K}_{d}(K)\right\}}$ has a unique maximum. For $\boldsymbol{X} \in \mathcal{U}_{d}(K)$, $\boldsymbol{X}^{\prime} \mid\{S=K\}$ admits a density through (5), and $\boldsymbol{x}^{\prime} \mapsto f_{\boldsymbol{X}^{\prime} \mid\{S=K\}}\left(\boldsymbol{x}^{\prime}\right)$ has a unique maximum attained by the mode of $\boldsymbol{X}^{\prime} \mid\{S=K\}$. By Proposition 2, elliptical random vectors with continuous and decreasing density generators form a subclass of $\mathcal{U}_{d}(K)$. Although some exchangeable random vectors possessing negative dependence, such as Model (b) in Section 2.2, may not be included in $\mathcal{U}_{d}(K)$, we believe that most loss models used in risk management practice are contained in $\mathcal{U}_{d}(K)$. As explained in Section 3.3, uniqueness of the mode of $\boldsymbol{X}^{\prime} \mid\{S=K\}$ and its unimodality are different concepts, and thus the class $\mathcal{U}_{d}(K)$ contains multimodal random vectors in the sense that the density $f_{\boldsymbol{X}^{\prime} \mid\{S=K\}}$ has multiple local maximizers (we call them the local modes of $\boldsymbol{X}^{\prime} \mid\{S=K\}$; see Definition 8 for their formal definitions). Nevertheless, in 
this section we focus only on the unique global maximizer of $f_{\boldsymbol{X}^{\prime} \mid\{S=K\}}$ (not on local ones) since unimodal distributions are the primary object to apply the MLA to. The multimodal case will then be revisited in Section 4.2. As we will demonstrate in Section 5 , multimodality can be detected by searching for the modes of $f_{\boldsymbol{X}^{\prime} \mid\{S=K\}}$.

In the following we define the unique mode of $\boldsymbol{X}^{\prime} \mid\{S=K\}$ as a risk allocation of $K$.

Definition 4 (Maximum likelihood allocation). For $K>0$ and $\boldsymbol{X} \in \mathcal{U}_{d}(K)$, the maximum likelihood allocation (MLA) on a set $\mathcal{K} \subseteq \mathcal{K}_{d}(K)$ is defined by

$$
\boldsymbol{K}_{\mathrm{M}}[\boldsymbol{X} ; \mathcal{K}]=\operatorname{argmax}\left\{f_{\boldsymbol{X}}(\boldsymbol{x}): \boldsymbol{x} \in \mathcal{K}\right\},
$$

provided the function $\boldsymbol{x} \mapsto f_{\boldsymbol{X}}(\boldsymbol{x}) \mathbf{1}_{\{\boldsymbol{x} \in \mathcal{K}\}}$ has a unique maximum. When $\mathcal{K}=\mathcal{K}_{d}(K)$, we call it the maximum likelihood allocation.

By (5), MLA of $K$ on $\mathcal{K}$ can be equivalently formulated as

$$
\boldsymbol{K}_{\mathrm{M}}[\boldsymbol{X} ; \mathcal{K}]=\operatorname{argmax}\left\{f_{\boldsymbol{X}^{\prime} \mid\{S=K\}}\left(\boldsymbol{x}^{\prime}\right):\left(\boldsymbol{x}^{\prime}, K-\mathbf{1}_{d^{\prime}}^{\top} \boldsymbol{x}^{\prime}\right) \in \mathcal{K}\right\} .
$$

By definition, MLA on $\mathcal{K} \subseteq \mathcal{K}_{d}(K)$ is an allocation of $K$ in the sense that it satisfies the full allocation property $\mathbf{1}_{d}^{\top} \boldsymbol{K}_{\mathrm{M}}[\boldsymbol{X} ; \mathcal{K}]=K$. We mainly study the case when $\mathcal{K}=\mathcal{K}_{d}(K)$. However, as we will see in Section 4.1.2 and Appendix F.2, the set $\mathcal{K}$ can be taken so that $\boldsymbol{K}_{\mathrm{M}}[\boldsymbol{X} ; \mathcal{K}]$ satisfies some desirable properties for a risk allocation principle.

\subsubsection{Properties of MLA}

We now investigate properties of MLA as a risk allocation principle. For desirable properties of risk allocation in the case when the capital $K$ is exogenously given as a constant, see Maume-Deschamps et al. (2016). By construction, $\boldsymbol{K}_{\mathrm{M}}[\boldsymbol{X} ; \mathcal{K}]$ always satisfies the full allocation property (1). The following proposition summarizes other desirable properties of MLA.

Proposition 5 (Properties of MLA). Suppose $K>0$ and $\boldsymbol{X} \in \mathcal{U}_{d}(K)$.

1. Translation invariance: $\boldsymbol{K}_{\mathrm{M}}\left[\boldsymbol{X}+\boldsymbol{c} ; \mathcal{K}_{d}\left(K+\mathbf{1}_{d}^{\top} \boldsymbol{c}\right)\right]=\boldsymbol{K}_{\mathrm{M}}\left[\boldsymbol{X} ; \mathcal{K}_{d}(K)\right]+\boldsymbol{c}$ for $\boldsymbol{c} \in \mathbb{R}^{d}$.

2. Positive homogeneity: $\boldsymbol{K}_{\mathrm{M}}\left[c \boldsymbol{X} ; \mathcal{K}_{d}(c K)\right]=c \boldsymbol{K}_{\mathrm{M}}\left[\boldsymbol{X} ; \mathcal{K}_{d}(K)\right]$ for $c>0$.

3. Symmetry: For $(i, j) \in\{1, \ldots, d\}, i \neq j$, let $\tilde{\boldsymbol{X}}$ be a d-dimensional random vector such that $\tilde{X}_{j}=X_{i}$, $\tilde{X}_{i}=X_{j}$ and $\tilde{X}_{k}=X_{k}, k \in\{1, \ldots, d\} \backslash\{i, j\}$. If $\boldsymbol{X} \stackrel{d}{=} \tilde{\boldsymbol{X}}$, then $\boldsymbol{K}_{\mathrm{M}}\left[\boldsymbol{X} ; \mathcal{K}_{d}(K)\right]_{i}=\boldsymbol{K}_{\mathrm{M}}\left[\boldsymbol{X} ; \mathcal{K}_{d}(K)\right]_{j}$, where $\boldsymbol{K}_{\mathrm{M}}\left[\boldsymbol{X} ; \mathcal{K}_{d}(K)\right]_{l}$ is the lth component of $\boldsymbol{K}_{\mathrm{M}}\left[\boldsymbol{X} ; \mathcal{K}_{d}(K)\right]$ for $l=1, \ldots, d$.

4. Continuity: Suppose $\boldsymbol{X}_{n}, \boldsymbol{X} \in \mathcal{U}_{d}(K)$ have densities $f_{n}$ and $f$ for $n=1,2, \ldots$, respectively. If $f_{n}$ is uniformly continuous and bounded for $n=1,2, \ldots$, and $\boldsymbol{X}_{n} \rightarrow \boldsymbol{X}$ weakly, then $\lim _{n \rightarrow \infty} \boldsymbol{K}_{\mathrm{M}}\left[\boldsymbol{X}_{n} ; \mathcal{K}_{d}(K)\right]$ $=\boldsymbol{K}_{\mathrm{M}}\left[\boldsymbol{X} ; \mathcal{K}_{d}(K)\right]$

Translation invariance states that a sure loss $c \in \mathbb{R}^{d}$ requires the same amount of risk allocation and the rest of the total capital is allocated to the random loss $\boldsymbol{X}$. Positive homogeneity means that, for a proportion $c>0,100 c \%$ of the loss $\boldsymbol{X}$ requires $100 c \%$ of the total capital $K$ and the resulting MLA of $c \boldsymbol{X}$ is $100 c \%$ of the allocation of $K$ to $\boldsymbol{X}$. Symmetry implies that, if exchanging two units does not change 
the distribution of the joint loss, then equal amounts of capitals are allocated to them. Finally, continuity ensures that if MLA is calculated based on an estimated model $f_{n}$ of $f$, then this estimate of MLA is close to the true MLA. Note that the assumption that $\boldsymbol{X}_{n}, n=1,2, \ldots$, and $\boldsymbol{X}$ belong to $\mathcal{U}_{d}(K)$ is esssential so that the MLAs of $\boldsymbol{X}_{n}, n=1,2, \ldots$, and $\boldsymbol{X}$ are well-defined.

Next we discuss properties that need to be considered separately.

\section{RORAC compatibility and core compatibility:}

RORAC compatibility and core compatibility are important properties of risk allocations since either of them characterizes Euler allocation; see Tasche (1995) and Denault (2001). However, the definitions of these properties are not meaningful when $K$ is exogenously given as a constant. Moreover, similar constraints as in core compatibility can be additionally imposed on $\mathcal{K}_{d}(K)$ so that the resulting MLA is core-compatible; see Appendix F.2 for details.

\section{Riskless asset:}

The riskless asset condition requires the sure loss $X_{j}=c_{j}$ a.s. for $c_{j} \in \mathbb{R}$ to be covered by the amount of allocated capital $c_{j}$. This property needs to be considered separately since in this case $\boldsymbol{X}$ does not admit a density. Suppose that $X_{j}=c_{j} \in \mathbb{R}$ a.s. for $j \in I \subseteq\{1, \ldots, d\}$ and that $\boldsymbol{X}_{-I}=\left(X_{j}, j \in\{1, \ldots, d\} \backslash I\right)$ admits a density $f_{\boldsymbol{X}_{-I}}$. Since

$$
\left(\boldsymbol{X}_{I}, \boldsymbol{X}_{-I}\right)\left|\{S=K\} \stackrel{\mathrm{d}}{=}\left(\boldsymbol{c}, \boldsymbol{X}_{-I}\right)\right|\left\{\mathbf{1}_{|-I|}^{\top} \boldsymbol{X}_{-I}=K-\mathbf{1}_{|I|}^{\top} \boldsymbol{c}\right\} \stackrel{\mathrm{d}}{=}\left(\boldsymbol{c}, \boldsymbol{X}_{-I} \mid\left\{\mathbf{1}_{|-I|}^{\top} \boldsymbol{X}_{-I}=K-\mathbf{1}_{|I|}^{\top} \boldsymbol{c}\right\}\right),
$$

any realization $\boldsymbol{x}$ of $\boldsymbol{X} \mid\{S=K\}$ satisfies $\boldsymbol{x}_{I}=\boldsymbol{c}$ and the likelihood of $\boldsymbol{x}$ is quantified through the value of the density $f_{\boldsymbol{X}_{-I} \mid\left\{\mathbf{1}_{|-I|}^{\top} \boldsymbol{X}_{-I}=K-\mathbf{1}_{|I|}^{\top} \boldsymbol{c}\right\}}\left(\boldsymbol{x}_{-I}\right)$. According to this discussion, a natural extension of the definition of MLA to such a random vector $\boldsymbol{X}$ is

$$
\boldsymbol{K}_{\mathrm{M}}\left[\boldsymbol{X} ; \mathcal{K}_{d}(K)\right]_{I}=\boldsymbol{c} \quad \text { and } \quad \boldsymbol{K}_{\mathrm{M}}\left[\boldsymbol{X} ; \mathcal{K}_{d}(K)\right]_{-I}=\boldsymbol{K}_{\mathrm{M}}\left[\boldsymbol{X}_{-I} ; \mathcal{K}_{|-I|}\left(K-\mathbf{1}_{|I|}^{\top} \boldsymbol{c}\right)\right] \text {, }
$$

which is compatible with the riskless asset property.

\section{Allocation under comonotonicity:}

Suppose $\boldsymbol{X}$ is a comonotone random vector with continuous and strictly increasing margins $F_{1}, \ldots, F_{d}$. As seen in Example 4, it holds that $\boldsymbol{X} \mid\{S=K\}=\left(F_{1}^{-1}\left(u^{*}\right), \ldots, F_{d}^{-1}\left(u^{*}\right)\right)$ a.s., where $u^{*} \in[0,1]$ is the unique solution of $\sum_{j=1}^{d} F_{j}^{-1}(u)=K$. According to the extended definition of MLA (13), we have that

$$
\boldsymbol{K}_{\mathrm{M}}\left(\boldsymbol{X} ; \mathcal{K}_{d}(K)\right)=\left(F_{1}^{-1}\left(u^{*}\right), \ldots, F_{d}^{-1}\left(u^{*}\right)\right)
$$

\subsubsection{Discussion on MLA}

We now discuss whether MLA is an appropriate risk allocation principle, and also compare MLA with Euler allocation. Here we define Euler allocation by $\mathbb{E}[\boldsymbol{X} \mid\{S=K\}]$, which are VaR contributions (2) with $K=\operatorname{VaR}_{p}(S)$ for some confidence level $p \in(0,1)$. As shown in Proposition 5, MLA possesses properties naturally required as an allocation such as translation invariance, positive homogeneity and riskless asset. Euler allocation also satisfies these properties since $\mathbb{E}\left[\boldsymbol{X}+\boldsymbol{c} \mid\left\{\mathbf{1}_{d}^{\top}(\boldsymbol{X}+\boldsymbol{c})=K+\mathbf{1}_{d}^{\top} \boldsymbol{c}\right\}\right]=\mathbb{E}\left[\boldsymbol{X} \mid\left\{\mathbf{1}_{d}^{\top} \boldsymbol{X}=\right.\right.$ 
$K\}]+\boldsymbol{c}$ for $\boldsymbol{c} \in \mathbb{R}^{d}$ (translation invariance), $\mathbb{E}\left[c \boldsymbol{X} \mid\left\{\mathbf{1}_{d}^{\top}(c \boldsymbol{X})=c K\right\}\right]=c \mathbb{E}\left[\boldsymbol{X} \mid\left\{\mathbf{1}_{d}^{\top} \boldsymbol{X}=K\right\}\right]$ for $c>0$ (positive homogeneity) and the riskless asset property holds by taking expectation on the both sides of the first equality in (12). See Appendix D for properties that neither MLA nor Euler allocation satisfy. Note that by Proposition 1 and Proposition 2 Part 1, Euler and maximum likelihood allocations coincide when $\boldsymbol{X}$ is elliptically distributed. Therefore, the economic justifications of Euler allocation, such as RORAC compatibility and core-compatibility, also hold for MLA when $\boldsymbol{X}$ is elliptical. Moreover, through the process of estimating a MLA, one can detect multimodality of $\boldsymbol{X}^{\prime} \mid\{S=K\}$ and discover hidden risky scenarios based on which one can evaluate the soundness of risk allocations. On the other hand, the main disadvantage of MLA compared with Euler allocation is that estimating modes becomes more difficult than estimating a mean as the dimension of the portfolio becomes larger. Furthermore, MLA is not well-defined for distributions whose $\operatorname{argmax}\left\{f_{\boldsymbol{X}}(\boldsymbol{x}): \boldsymbol{x} \in \mathcal{K}_{d}(K)\right\}$ is not a single point. Finally, MLA may ignore the behavior of $\boldsymbol{X} \mid\{S=K\}$ other than its mode. Considering these aspects, we believe that MLA itself may not be an appropriate risk allocation principle, but its estimation procedure is beneficial for discovering hidden multiple scenarios likely to occur in the stressed situation $\{S=K\}$, for assessing the soundness of risk allocations in stress testing applications, and eventually for constructing more sound risk allocations based on multiple scenarios as we will consider in Section 4.2.

\subsection{Multimodality adjustment of risk allocations}

Multiple local modes of $\boldsymbol{X} \mid\{S=K\}$ can be discovered in the process of estimating MLAs. In this section, we discuss how to utilize the local modes, and introduce the so-called multimodality adjustment to increase the soundness of risk allocations under multimodality of $\boldsymbol{X} \mid\{S=K\}$.

\subsubsection{Definition of multimodality adjustment}

Suppose that $M \in \mathbb{N}$ scenarios $\boldsymbol{K}_{1}, \ldots, \boldsymbol{K}_{M} \in \mathcal{K}_{d}(K)$ are found with corresponding probability weights $w_{1}, \ldots, w_{M} \in[0,1]$ such that $\sum_{m=1}^{M} w_{m}=1$. A typical choice of the scenario set $\left\{\boldsymbol{K}_{1}, \ldots, \boldsymbol{K}_{M}\right\}$ is the set of local modes of $\boldsymbol{x} \mapsto f_{\boldsymbol{X}}(\boldsymbol{x}) \mathbf{1}_{\left\{\boldsymbol{x} \in \mathcal{K}_{d}(K)\right\}}$ (assumed to be a finite set), or possibly those belonging to a superlevel set at a certain level of plausibility. The probability weight $w_{m}$ typically represents the likelihood of the scenario $\boldsymbol{K}_{m}$ to occur, for instance, $w_{m} \propto f_{\boldsymbol{X}}\left(\boldsymbol{K}_{m}\right) / f_{S}(K)$. Moreover, experts' assessments of the impact of the loss $\boldsymbol{K}_{m}$ on the portfolio $\boldsymbol{X}$ can also be incorporated. Multimodality adjustment is then defined as follows.

Definition 5 (Multimodality adjustment of risk allocations). Let $M \in \mathbb{N}$ be the number of scenarios, $\mathcal{X}=\left\{\boldsymbol{K}_{1}, \ldots, \boldsymbol{K}_{M}\right\}$ be the set of scenarios where $\boldsymbol{K}_{m} \neq \boldsymbol{K}_{m^{\prime}}$ for any $m, m^{\prime} \in\{1, \ldots, M\}$ such that $m \neq m^{\prime}$, and $\boldsymbol{w}=\left(w_{1}, \ldots, w_{M}\right)$ be the associated probability weights such that $\sum_{m=1}^{M} w_{m}=1$. Then the multimodality-adjusted allocated capital is defined by

$$
\boldsymbol{K}_{\boldsymbol{w}, \mathcal{X}, \Lambda}=\overline{\boldsymbol{K}}_{\boldsymbol{w}, \mathcal{X}}+\sum_{m=1}^{M} w_{m} \boldsymbol{\lambda}_{m} \circ\left(\boldsymbol{K}_{m}-\overline{\boldsymbol{K}}_{\boldsymbol{w}, \mathcal{X}}\right)^{+},
$$

where $\overline{\boldsymbol{K}}_{\boldsymbol{w}, \mathcal{X}}=\sum_{m=1}^{M} w_{m} \boldsymbol{K}_{m}$ is the baseline allocation, $\Lambda=\left(\boldsymbol{\lambda}_{1}, \ldots, \boldsymbol{\lambda}_{M}\right) \in \mathbb{R}_{+}^{d \times M}$ is the matrix of multimodality loading parameters, $\boldsymbol{x} \circ \boldsymbol{y}=\left(x_{1} y_{1}, \ldots, x_{d} y_{d}\right)$ for $\boldsymbol{x}, \boldsymbol{y} \in \mathbb{R}^{d}$ and $\boldsymbol{x}^{+}=\left(\max \left(x_{1}, 0\right), \ldots, \max \left(x_{d}, 0\right)\right)$ for $\boldsymbol{x} \in \mathbb{R}^{d}$. We call the second term $\sum_{m=1}^{M} w_{m} \boldsymbol{\lambda}_{m} \circ\left(\boldsymbol{K}_{m}-\overline{\boldsymbol{K}}_{\boldsymbol{w}, \mathcal{X}}\right)^{+}$of (14) the multimodality adjustment. 
Unlike MLA, the multimodality-adjusted allocated capital can be well-defined even if the global mode of $\boldsymbol{X} \mid\{S=K\}$ is not unique. The capital (14) consists of the baseline allocation and the additional loading to cover the variability of scenarios. The baseline allocation is understood as an allocated capital before adjustment of multimodality. Therefore, $\overline{\boldsymbol{K}}_{\boldsymbol{w}, \mathcal{X}}$ in (14) can be replaced by Euler allocation if one requires its economic justification such as RORAC compatibility and core-compatibility. To explain the multimodality adjustment, suppose that the scenario $\left\{\boldsymbol{X}=\boldsymbol{K}_{m}\right\}$ occurs with probability $w_{m}$. Under this scenario, the portfolio incurs the loss (or profit) $\boldsymbol{K}_{m}-\overline{\boldsymbol{K}}_{\boldsymbol{w}, \mathcal{X}}$. When $\boldsymbol{\lambda}_{m}=\mathbf{1}_{d}$, the actual amount of loss $\left(\boldsymbol{K}_{m}-\overline{\boldsymbol{K}}_{\boldsymbol{w}, \mathcal{X}}\right)^{+}$contributes to the multimodality adjustment $\sum_{m=1}^{M} w_{m} \boldsymbol{\lambda}_{m} \circ\left(\boldsymbol{K}_{m}-\overline{\boldsymbol{K}}_{\boldsymbol{w}, \mathcal{X}}\right)^{+}$. However, this choice of $\boldsymbol{\lambda}_{m}$ is too conservative, and smaller values of $\boldsymbol{\lambda}_{m}$ are typically more reasonable since both $\boldsymbol{K}_{m}$ and $\overline{\boldsymbol{K}}_{\boldsymbol{w}, \mathcal{X}}$ sum up to $K$ and thus losses of some units imply profits of others. Therefore, losses of some units can be compensated by the profits of other units, and the multimodality loading parameter $\boldsymbol{\lambda}_{m}$ can be determined by such risk mitigation or a corresponding insurance contract.

\subsubsection{Properties of the multimodality adjustment}

Next we study properties of $\boldsymbol{K}_{\boldsymbol{w}, \mathcal{X}, \Lambda}$. For certain choices of $\boldsymbol{w}$ and $\mathcal{X}$, the capital $\boldsymbol{K}_{\boldsymbol{w}, \mathcal{X}, \Lambda}$ can be shown to satisfy translation invariance, positive homogeneity, riskless asset and symmetry; see Appendix E for details. In the remainder of this section, we will verify that $\boldsymbol{K}_{\boldsymbol{w}, \mathcal{X}, \Lambda}$ measures the risk of multimodality from various viewpoints. First, if $M=1$, then $\boldsymbol{K}_{\boldsymbol{w}, \mathcal{X}, \Lambda}=\overline{\boldsymbol{K}}_{\boldsymbol{w}, \mathcal{X}}$ and thus the multimodality adjustment is zero. Second, suppose that $M \geq 2$ and $w_{m}>0$ for $m=1, \ldots, M$. Then $\boldsymbol{K}_{\boldsymbol{w}, \mathcal{X}, \Lambda}=\overline{\boldsymbol{K}}_{\boldsymbol{w}, \mathcal{X}}$ if and only if $\lambda_{j, m}=0$ for all $j=1, \ldots, d$ and $m=1, \ldots, M$ such that $K_{m, j}>\bar{K}_{\boldsymbol{w}, \mathcal{X}, j}$. Therefore, under multimodality, the multimodality adjustment is zero if and only if losses of some units of the portfolio are completely compensated by profits of others. Finally, $\boldsymbol{K}_{\boldsymbol{w}, \mathcal{X}, \Lambda}$ is increasing with respect to the variability of the set of scenarios, which can be understood as a degree of multimodality. To see this, suppose that $\boldsymbol{\lambda}_{1}=\cdots=\boldsymbol{\lambda}_{M}=\boldsymbol{\lambda}$ for some $\boldsymbol{\lambda} \in \mathbb{R}_{+}^{d}$, and denote by $\boldsymbol{Y}$ the discrete random vector taking points $\boldsymbol{K}_{1}, \ldots, \boldsymbol{K}_{M}$ with probabilities $w_{1}, \ldots, w_{M}$. Then the multimodality-adjusted allocated capital (14) can be written as

$$
\boldsymbol{K}_{\boldsymbol{w}, \mathcal{X}, \Lambda}=\mathbb{E}[\boldsymbol{Y}]+\boldsymbol{\lambda} \circ \mathbb{E}\left[(\boldsymbol{Y}-\mathbb{E}[\boldsymbol{Y}])^{+}\right]
$$

Variability of the set of scenarios can then be compared by the so-called convex order of $Y_{1}, \ldots, Y_{d}$. For two $\mathbb{R}$-valued random variables $Y$ and $Y^{\prime}, Y^{\prime}$ is said to be larger than $Y$ in the convex order, denoted as $Y \leq_{\mathrm{cx}} Y^{\prime}$, if $\mathbb{E}[\phi(Y)] \leq \mathbb{E}\left[\phi\left(Y^{\prime}\right)\right]$ for all convex functions $\phi: \mathbb{R} \rightarrow \mathbb{R}$ provided the expectations exist; see Shaked and Shanthikumar (2007) for a comprehensive reference. Roughly speaking, convex order compares the variability of random variables and $Y^{\prime}$ shows more variability than $Y$ if $Y \leq_{\mathrm{cx}} Y^{\prime}$; for instance, $Y \leq_{\mathrm{cx}} Y^{\prime}$ implies $\mathbb{E}[Y]=\mathbb{E}\left[Y^{\prime}\right], \operatorname{Var}(Y) \leq \operatorname{Var}\left(Y^{\prime}\right)$, ess.inf $\left(Y^{\prime}\right) \leq \operatorname{ess} \cdot \inf (Y)$, ess.sup $(Y) \leq \operatorname{ess.sup}\left(Y^{\prime}\right)$ and $\mathbb{E}[(Y-$ $\left.a)^{+}\right] \leq \mathbb{E}\left[\left(Y^{\prime}-a\right)^{+}\right]$for all $a \in \mathbb{R}$. Therefore, for two sets of scenarios $\mathcal{X}$ and $\mathcal{X}^{\prime}$ with associated probabilities $\boldsymbol{w}$ and $\boldsymbol{w}^{\prime}$, if one shows more variability than the other in the sense that the corresponding discrete random variables satisfy $Y_{j} \leq_{\text {cx }} Y_{j}^{\prime}$ for some $j \in\{1, \ldots, d\}$, then it holds that $\left(\boldsymbol{K}_{\boldsymbol{w}, \mathcal{X}, \Lambda}\right)_{j} \leq\left(\boldsymbol{K}_{\boldsymbol{w}^{\prime}, \mathcal{X}^{\prime}, \Lambda}\right)_{j}$.

Remark 4 (Multimodality adjustment for general sets of scenarios). Representation (15) bears structural resemblance to Gini shortfall allocations introduced in Furman et al. (2017), and (15) indicates a possible extension of the multimodality adjustment to the case when the set of scenarios is not finite. For instance, 
by taking $\mathcal{X}=\left\{\boldsymbol{x} \in \mathbb{R}^{d}: \mathbf{1}_{d}^{\top} \boldsymbol{x} \geq \operatorname{VaR}_{p}(S)\right\}$ and $w(\boldsymbol{x})=f_{\boldsymbol{X} \mid\left\{S \geq \operatorname{VaR}_{p}(S)\right\}}(\boldsymbol{x})$, (15) can be interpreted as multimodality-adjusted Euler allocations of Expected Shortfall since (15) yields

$$
\boldsymbol{K}_{\boldsymbol{w}, \mathcal{X}, \Lambda}=\mathrm{ES}_{p}\left(X_{j} ; S\right)+\boldsymbol{\lambda} \circ \mathbb{E}\left[\left(\boldsymbol{X}-\mathrm{ES}_{p}\left(X_{j} ; S\right)\right)^{+} \mid\left\{S \geq \operatorname{VaR}_{p}(S)\right\}\right],
$$

where $\operatorname{ES}_{p}\left(X_{j} ; S\right)=\mathbb{E}\left[\boldsymbol{X} \mid\left\{S \geq \operatorname{VaR}_{p}(S)\right\}\right]$ is the Euler allocation of $K=\operatorname{ES}_{p}(S)$ as derived in (3).

We end this section with a remark on the case when multiple measures or models are considered as different scenarios and how to incorporate these scenarios into multimodality-adjusted allocated capitals.

Remark 5 (Multimodality adjustment for different measures). A single model of a risk may not be sufficient to manage the risk due to changes of an economic situation or due to model uncertainty. For a further risk assessment, it may be useful to consider multiple measures $\mathbb{Q}_{1}, \ldots, \mathbb{Q}_{S}$ where $\mathbb{Q}_{s}$ is a probability measure on $(\Omega, \mathcal{A})$ and $F_{\boldsymbol{X}}^{\mathbb{Q}_{s}}$ is the distribution function of $\boldsymbol{X}$ under $\mathbb{Q}_{s}$ for $s=1, \ldots, S$. These multiple measures can be incorporated into the scenario analysis by, for example, considering the (componentwise) maximum of the multimodality-adjusted allocated capitals $\mathcal{K}_{\boldsymbol{w}, \mathcal{X}, \Lambda}$ calculated based on $F_{\boldsymbol{X}}^{\mathbb{Q}_{s}}$ for $s=1, \ldots, S$, or considering their mixture with respect to probabilities $q_{1}, \ldots, q_{S}$ where $q_{s}$ is associated to the scenario $\mathbb{Q}_{s}$ determined, for example, proportionally to the sample size available for the distribution $F_{\boldsymbol{X}}^{\mathbb{Q}_{s}}$.

\section{$5 \quad$ Numerical experiments}

In this section we conduct an empirical and simulation study to compute Euler and maximum likelihood allocations, and compare them for various models. Simulation of the conditional distribution given a constant sum is in general challenging. Throughout this section, we adopt (crude) Monte Carlo (MC) method to simulate $\boldsymbol{X}^{\prime} \mid\{S=K\}$ according to which unconditional samples from $\boldsymbol{X}$ are first generated and those falling in the region $\mathcal{K}_{d}(K, \delta)=\left\{\boldsymbol{x} \in \mathbb{R}^{d}: K-\delta<\mathbf{1}_{d}^{\top} \boldsymbol{x}<K+\delta\right\}$ for a sufficiently small $\delta>0$ are then extracted. The extracted samples are standardized via $K X_{j} / \sum_{j=1}^{d} X_{j}$ so that their componentwise sum equals $K$. Finally the standardized samples are used as pseudo-samples from $\boldsymbol{X}^{\prime} \mid\{S=K\}$. See Appendix F.1 for the potential bias produced by this method, and more sophisticated simulation approaches of $\boldsymbol{X}^{\prime} \mid\{S=K\}$ based on MCMC methods. All experiments are run on a MacBook Air with 1.4 GHz Intel Core i5 processor and 4 GB $1600 \mathrm{MHz}$ of DDR3 RAM.

\subsection{Empirical study}

In this section we estimate MLA nonparametrically for real financial data. We consider daily log-returns of the stock indices FTSE $X_{t, 1}$, S\&P $500 X_{t, 2}$ and DJI $X_{t, 3}$ from January 2, 1990 to March 25, 2004, which contains 3713 days and thus $T=3712 \log$-returns. We consider two portfolios (a) $\boldsymbol{X}_{t}^{\text {pos }}=\left(X_{t, 1}, X_{t, 2}, X_{t, 3}\right)$ and (b) $\boldsymbol{X}_{t}^{\text {neg }}=\left(X_{t, 1},-X_{t, 2}, X_{t, 3}\right)$. For each portfolio, we aim at allocating the capital $K=1$ based on the conditional loss distribution at time $T+1$ given the history up to and including time $T$. Taking into account the stylized facts of stock returns listed in Chapter 3 of McNeil et al. (2015) (such as unimodality, heavytailedness and volatility clusters), we adopt a copula-GARCH model with marginal skew- $t$ innovations (ST-GARCH; see, for example, Jondeau and Rockinger (2006) and Huang et al. (2009)). We utilize a $\operatorname{GARCH}(1,1)$ model with skew- $t$ innovations with degrees of freedom $\nu_{j}>0$ and skewness parameter $\gamma_{j}>0$ for the $j$ th marginal time series. That is, within a fixed time period $\{1, \ldots, T+1\}$, the $j$ th return 
Table 1: Maximum likelihood estimates and estimated standard errors of the ST-GARCH $(1,1)$ model $X_{t, j}=$ $\mu_{j}+\sigma_{t, j} Z_{t, j}$ with $\sigma_{t, j}^{2}=\omega_{j}+\alpha_{j} X_{t-1, j}^{2}+\beta_{j} \sigma_{t-1, j}^{2}$ and $Z_{t, j} \stackrel{\text { iid }}{\sim} \operatorname{ST}\left(\nu_{j}, \gamma_{j}\right)$ for $j=1,2,3$.

\begin{tabular}{lcccccc}
\hline & $\mu_{j}$ & $\omega_{j}$ & $\alpha_{j}$ & $\beta_{j}$ & $\gamma_{j}$ & $\nu_{j}$ \\
\hline$X_{t, 1}^{\text {pos }}=X_{t, 1}^{\text {neg }}$ & 0.053 & 0.006 & 0.052 & 0.943 & 0.969 & 6.414 \\
$\mathrm{SE}$ & 0.013 & 0.002 & 0.008 & 0.008 & 0.021 & 0.663 \\
$X_{t, 2}^{\text {pos }}$ & 0.050 & 0.003 & 0.049 & 0.950 & 0.983 & 6.265 \\
$\mathrm{SE}$ & 0.013 & 0.001 & 0.007 & 0.007 & 0.021 & 0.659 \\
$X_{t, 2}^{\text {neg }}$ & -0.050 & 0.003 & 0.049 & 0.950 & 1.018 & 6.265 \\
$\mathrm{SE}$ & 0.013 & 0.001 & 0.007 & 0.007 & 0.022 & 0.659 \\
$X_{t, 3}^{\text {pos }}=X_{t, 3}^{\text {neg }}$ & 0.031 & 0.011 & 0.071 & 0.920 & 0.966 & 10.000 \\
$\mathrm{SE}$ & 0.014 & 0.003 & 0.009 & 0.010 & 0.023 & 1.309 \\
\hline
\end{tabular}

series $\left(X_{1, j}, \ldots, X_{T+1, j}\right)$ follows

$$
X_{t, j}=\mu_{j}+\sigma_{t, j} Z_{t, j}, \quad \sigma_{t, j}^{2}=\omega_{j}+\alpha_{j} X_{t-1, j}^{2}+\beta_{j} \sigma_{t-1, j}^{2}, \quad Z_{t, j} \stackrel{\mathrm{iid}}{\sim} \operatorname{ST}\left(\nu_{j}, \gamma_{j}\right), \quad j=1, \ldots, d,
$$

where $\omega_{j}>0, \alpha_{j}, \beta_{j} \geq 0, \alpha_{j}+\beta_{j}<1$, and $Z_{t, j}$ follows a skew- $t$ distribution $\operatorname{ST}\left(\nu_{j}, \gamma_{j}\right)$ with density given by

$$
f_{j}\left(x_{j} ; \nu_{j}, \gamma_{j}\right)=\frac{2}{\gamma_{j}+\frac{1}{\gamma_{j}}}\left\{t\left(x_{j}, \nu_{j}\right) 1_{\left[x_{j} \geq 0\right]}+t\left(\gamma_{j} x_{j}, \nu_{j}\right) 1_{\left[x_{j}<0\right]}\right\}
$$

where $t(x, \nu)$ is the density function of a Student $t$ distribution with degrees of freedom $\nu>0$ and a skewness parameter $\gamma>0$ with $\gamma=1$ corresponding to the standard symmetric case; see Fernández and Steel (1998) for more details. The copula among the stationary process $\boldsymbol{Z}_{t}=\left(Z_{t, 1}, \ldots, Z_{t, d}\right)$, denoted as $C$, is estimated nonparametrically. Under this model, the joint distribution of the returns $\boldsymbol{X}_{T+1 \mid \mathcal{F}_{T}}=$ $\left(X_{T+1,1 \mid \mathcal{F}_{T}}, \ldots, X_{T+1, d \mid \mathcal{F}_{T}}\right)$ has marginal distributions $\mathrm{ST}\left(\mu_{j}, \sigma_{t+1, j}^{2}, \nu_{j}, \gamma_{j}\right), j=1, \ldots, d$, and a copula $C$, where $\operatorname{ST}\left(\mu_{j}, \sigma_{t+1, j}^{2}, \nu_{j}, \gamma_{j}\right)$ is a skew- $t$ distribution with density $f_{j}\left(\frac{x_{j}-\mu_{j}}{\sigma_{t+1, j}} ; \nu_{j}, \gamma_{j}\right)$ with $f_{j}\left(\cdot ; \nu_{j}, \gamma_{j}\right)$ defined in (16). Parameters of the ST-GARCH $(1,1)$ models are estimated with the maximum likelihood method; the results are summarized in Table 1.

For each case of (a) $\boldsymbol{X}^{\text {pos }}$ and (b) $\boldsymbol{X}^{\text {neg }}$, we estimate the Euler allocation and MLA by a resampling method. After extracting the marginal standardized residuals, we build their pseudo-observations as a pseudo-sample from $C$. We then generate samples of size $N=3712$ by resampling with replacement. The samples from $C$ are then marginally transformed by skew- $t$ distributions with parameters specified as in Table 1. From these samples of $\boldsymbol{X}_{T+1 \mid \mathcal{F}_{T}}$, we extract the subsamples falling in the region $\mathcal{K}_{d}(K, \delta)=\left\{\boldsymbol{x} \in \mathbb{R}^{3}: K-\delta<\sum_{j=1}^{3} x_{j}<K+\delta\right\}$ where $\delta=0.3$. These samples are then standardized via $K X_{t, j} / \sum_{j=1}^{d} X_{t, j}$. Scatter plots of the first two components of these samples are shown in Figure 2.

The 3712 data points lead to 354 and 558 samples from $\boldsymbol{X}_{T+1 \mid \mathcal{F}_{T}}^{\text {pos }}$ and $\boldsymbol{X}_{T+1 \mid \mathcal{F}_{T}}^{\text {neg }}$ on $\mathcal{K}_{d}(K, \delta)$, respectively. Based on these conditional samples, we estimate the Euler allocation $\mathbb{E}[\boldsymbol{X} \mid\{S=K\}]$ and the MLA, that is, the mode of $f_{\boldsymbol{X} \mid\{S=K\}}$ provided it is unique. The (possibly multiple) modes were estimated by the function kms (kernel mean shift clustering, proposed by Fukunaga and Hostetler, 1975) of the R package ks; 
(a)

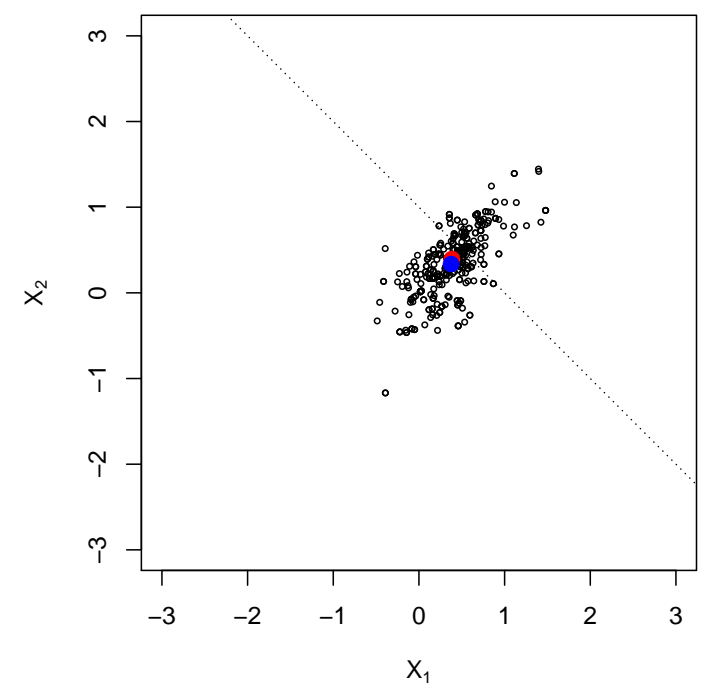

(b)

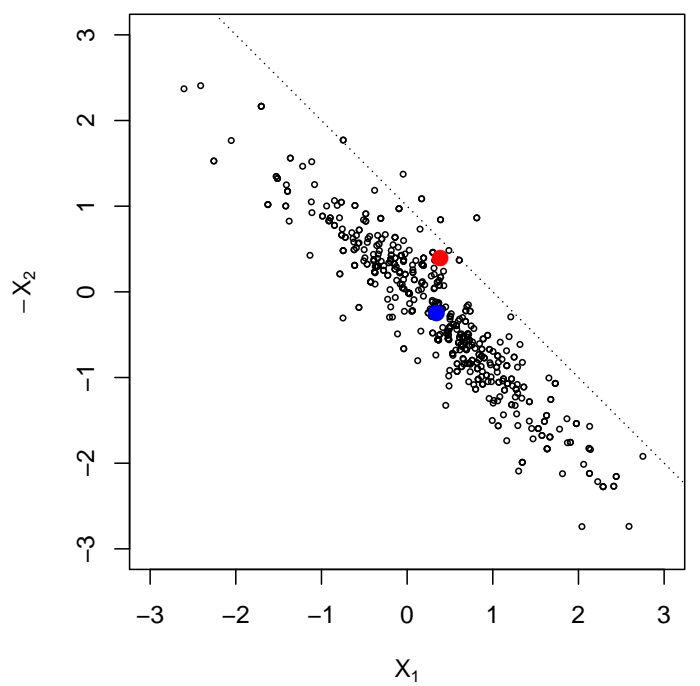

Figure 2: Scatter plots (black dots) of the first two components of (a) $\boldsymbol{X}_{T+1 \mid \mathcal{F}_{T}}^{\text {pos }}=$ $\left(X_{T+1,1 \mid \mathcal{F}_{T}}, X_{T+1,2 \mid \mathcal{F}_{T}}, X_{T+1,3 \mid \mathcal{F}_{T}}\right)$ and (b) $\boldsymbol{X}_{T+1 \mid \mathcal{F}_{T}}^{\text {neg }}=\left(X_{T+1,1 \mid \mathcal{F}_{T}},-X_{T+1,2 \mid \mathcal{F}_{T}}, X_{T+1,3 \mid \mathcal{F}_{T}}\right)$ for daily logreturns of the stock indices FTSE $X_{t, 1}$, S\&P $500 X_{t, 2}$ and DJI $X_{t, 3}$ falling in the region $\mathcal{K}_{d}(K, \delta)=$ $\left\{\boldsymbol{x} \in \mathbb{R}^{3}: K-\delta<\sum_{j=1}^{3} x_{j}<K+\delta\right\}$ where $\delta=0.3$ and $K=1$. The dotted lines represent $x+y=K$. The red dot represents the Euler allocation $\mathbb{E}\left[\boldsymbol{X}^{\prime} \mid\{S=K\}\right]$ and the blue dot represents the maximum likelihood allocation, which is the mode of $f_{\boldsymbol{X}^{\prime} \mid\{S=K\}}$.

see Carreira-Perpinán (2015) and Chen et al. (2016) for details and for other methods of estimating modes. For the computational times, computing MLAs took 0.35 seconds in Case (a) and 0.43 seconds in Case (b) whereas, in both cases, the Euler allocations were computed almost instantly. As was expected from the ellipticality of the scatter plots in Figure 2, the unique mode was discovered in each case. The first two components of the two allocations are pointed out in Figure 2.

Next, we estimate the standard errors of the Euler and maximum likelihood allocations using the bootstrap method. We compute the Euler allocation, MLA and their standard errors based on the $B=100$ number of samples of size $N=3712$ resampled from the original data with replacement. The results are summarized in Table 2.

In Figure 2 we can observe that compared with Case (a) the distribution in Case (b) is more spread out and losses take larger absolute values. If the samples are regarded as stressed scenarios, the scenario set in Case (b) contains a wider variety of scenarios than in Case (a) since both of positive and negative losses can appear in Case (b) whereas most realizations are positive in Case (a). Nevertheless, as is observed from Table 2, in both cases the Euler allocation and the MLA are close to each other also in terms of standard errors. This observation does not conflict with the stylized fact that the joint log-returns nearly follow an elliptical distribution, and thus the mean (Euler allocation) of $\boldsymbol{X} \mid\{S=K\}$ coincides with its mode; see Proposition 1 and Proposition 2 Part 1. 
Table 2: Bootstrap estimates and estimated standard errors of the Euler allocation and MLA of $\boldsymbol{X}_{T+1 \mid \mathcal{F}_{T}}^{\text {pos }}=$ $\left(X_{T+1,1 \mid \mathcal{F}_{T}}, X_{T+1,2 \mid \mathcal{F}_{T}}, X_{T+1,3 \mid \mathcal{F}_{T}}\right)$ and $\boldsymbol{X}^{\mathrm{neg}}=\left(X_{T+1,1 \mid \mathcal{F}_{T}},-X_{T+1,2 \mid \mathcal{F}_{T}}, X_{T+1,3 \mid \mathcal{F}_{T}}\right)$ for daily log-returns of the stock indices FTSE $X_{t, 1}$, S\&P $500 X_{t, 2}$ and DJI $X_{t, 3}$. The subsample size is $N=3712$ and the bootstrap sample size is $B=100$.

\begin{tabular}{lcccccccc}
\hline & \multicolumn{3}{c}{ Estimator } & & \multicolumn{3}{c}{ Standard error } \\
\cline { 2 - 3 } \cline { 7 - 9 } & $X_{1}$ & $X_{2}$ & $X_{3}$ & & $X_{1}$ & $X_{2}$ & $X_{3}$ \\
\hline $\mathbb{E}\left[\boldsymbol{X}^{\text {pos }} \mid\{S=K\}\right]$ & 0.378 & 0.338 & 0.285 & & 0.019 & 0.022 & 0.038 \\
$\boldsymbol{K}_{\mathrm{M}}\left[\boldsymbol{X}^{\text {pos }} ; \mathcal{K}_{d}(K)\right]$ & 0.367 & 0.365 & 0.268 & & 0.019 & 0.024 & 0.041 \\
$\mathbb{E}\left[\boldsymbol{X}^{\text {neg }} \mid\{S=K\}\right]$ & 0.345 & -0.248 & 0.903 & & 0.037 & 0.039 & 0.015 \\
$\boldsymbol{K}_{\mathrm{M}}\left[\boldsymbol{X}^{\text {neg }} ; \mathcal{K}_{d}(K)\right]$ & 0.371 & -0.280 & 0.909 & & 0.040 & 0.039 & 0.013 \\
\hline
\end{tabular}

\subsection{Simulation study}

In this section, we consider four models, referred to as (M1), (M2), (M3) and (M4), respectively, with $d=3$ and having the same marginal distributions $X_{1} \sim \operatorname{Par}(2.5,5), X_{2} \sim \operatorname{Par}(2.75,5)$ and $X_{3} \sim \operatorname{Par}(3,5)$ (where $\operatorname{Par}(\theta, \lambda)$ denotes the Pareto distribution with shape parameter $\theta>0$ and scale parameter $\lambda>0$ ) but different $t$ copulas with degrees of freedom $\nu=5$ and dispersion matrices

$$
\begin{aligned}
& P_{1}=\left(\begin{array}{ccc}
1 & 0.8 & 0.5 \\
0.8 & 1 & 0.8 \\
0.5 & 0.8 & 1
\end{array}\right), \quad P_{2}=\left(\begin{array}{ccc}
1 & 0.5 & 0.5 \\
0.5 & 1 & 0.5 \\
0.5 & 0.5 & 1
\end{array}\right) \\
& P_{3}=\left(\begin{array}{ccc}
1 & 0 & 0.5 \\
0 & 1 & 0 \\
0.5 & 0 & 1
\end{array}\right), \quad P_{4}=\left(\begin{array}{ccc}
1 & -0.5 & 0.5 \\
-0.5 & 1 & -0.5 \\
0.5 & -0.5 & 1
\end{array}\right) \text {, }
\end{aligned}
$$

respectively. For these parametric models, we first simulate $N=10^{6}$ samples from the unconditional distribution and then extract subsamples falling in the region $\mathcal{K}_{d}(K, \delta)$ with $K=40$ and $\delta=1$. These pseudo samples from $\boldsymbol{X}^{\prime} \mid\{S=K\}$ are shown in Figure 3. The red point in the figure represents the Euler allocation and the blue points are the (local) modes, which are estimated similarly as in Section 5.1.

The computational times required for calculating MLAs were (in seconds) (M1) 18.96, (M2) 11.72, (M3) 15.94, and (M4) 22.76 whereas the Euler allocations were computed almost instantly for all the cases. Compared with the results in Section 5.1, we observe that the computational time required to calculate MLA increases more rapidly than the Euler allocation does as the sample size increases.

In Figure 3 we can observe that the conditional distribution is more concentrated under positive dependence (Model (M1) and (M2)) and it is more dispersed under negative dependence (Model (M4)). Regarding the samples as stressed scenarios, the sets for Model (M3) and (M4) are more worrisome than those for Model (M1) and (M2) since (M3) and (M4) contain two distinct scenarios, one around the first axis and another around the upper-left corner of the plot region, both of which are likely to occur in the stressed situation $\{S=K\}$. Unimodality of the conditional distribution for Model (M1) and (M2) leads to closer Euler allocation and MLA. For Model (M1) and (M2), the choice of Euler allocation and MLA does not 
(M1)

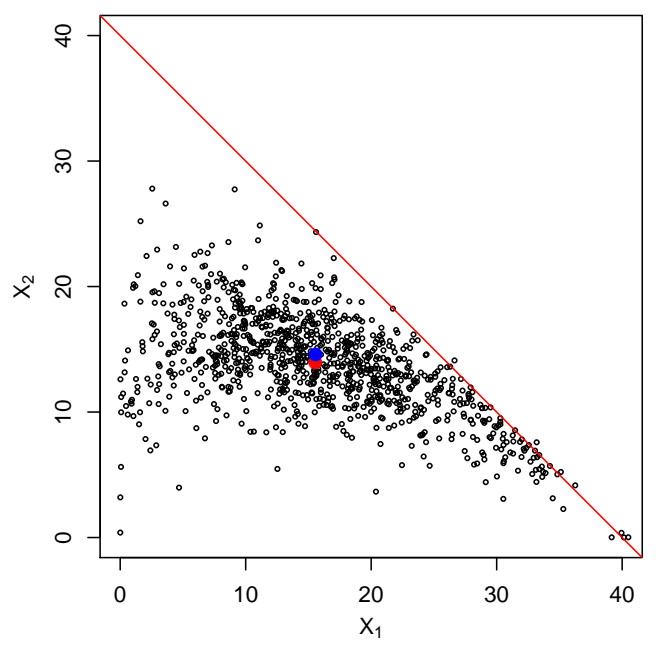

(M3)

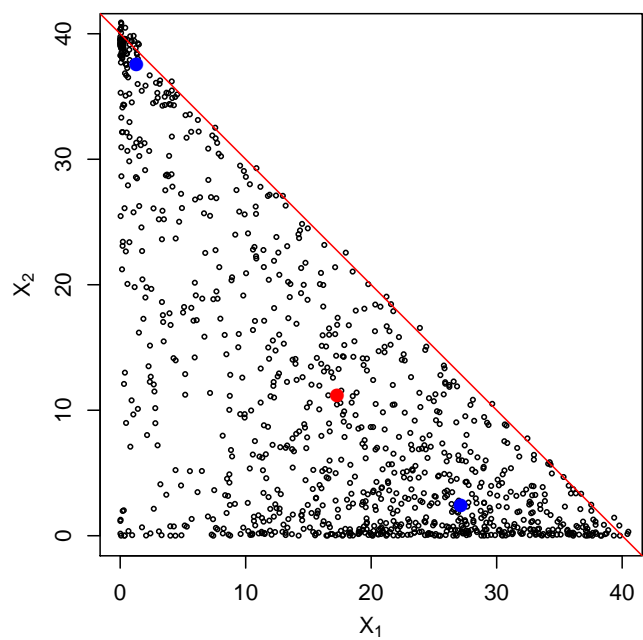

(M2)

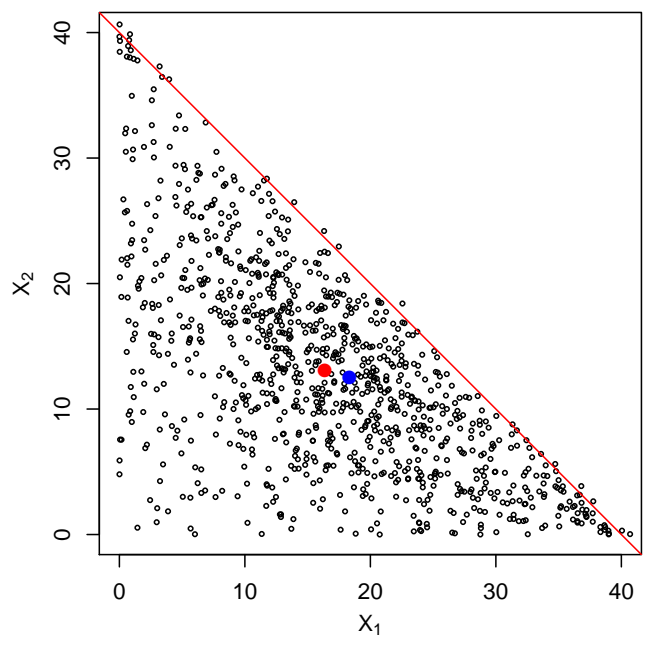

(M4)

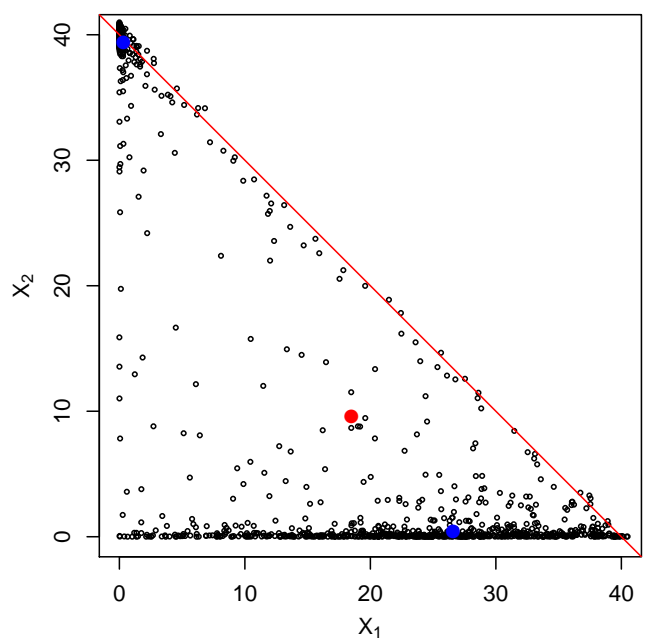

Figure 3: Scatter plots (black dots) of the first two components of the four models (M1), (M2), (M3) and (M4) falling in the region $\mathcal{K}_{d}(K, \delta)$ with $K=40$ and $\delta=1$. All the four models have the same marginal distributions $X_{1} \sim \operatorname{Par}(2.5,5), X_{2} \sim \operatorname{Par}(2.75,5)$ and $X_{3} \sim \operatorname{Par}(3,5)$ but different $t$ copulas with parameters provided in (17). The red lines represent $x+y=K$. The red dot represents the Euler allocation $\mathbb{E}\left[\boldsymbol{X}^{\prime} \mid\{S=K\}\right]$ and the blue dots represent the (local) modes of $f_{\boldsymbol{X}^{\prime} \mid\{S=K\}}$. 
Table 3: Estimates and estimated standard errors of the Euler allocation and MLA of the four models (M1), (M2), (M3) and (M4) all having the same marginal distributions $X_{1} \sim \operatorname{Par}(2.5,5), X_{2} \sim \operatorname{Par}(2.75,5)$ and $X_{3} \sim \operatorname{Par}(3,5)$ but different $t$ copulas with parameters provided in (17). Estimates and estimated standard errors are computed based on 100 replications, each of which consists of 500 conditional samples falling in the region $\mathcal{K}_{d}(K, \delta)$ with $K=40$ and $\delta=1$.

\begin{tabular}{|c|c|c|c|c|c|c|}
\hline & \multicolumn{3}{|c|}{ Estimator } & \multicolumn{3}{|c|}{ Standard error } \\
\hline & $X_{1}$ & $X_{2}$ & $X_{3}$ & $X_{1}$ & $X_{2}$ & $X_{3}$ \\
\hline \multicolumn{7}{|c|}{ (M1) Pareto $+t$ copula: strong positive dependence } \\
\hline $\mathbb{E}[\boldsymbol{X} \mid\{S=K\}]$ & 15.549 & 13.889 & 10.562 & 0.336 & 0.157 & 0.288 \\
\hline $\boldsymbol{K}_{\mathrm{M}}\left[\boldsymbol{X} ; \mathcal{K}_{d}(K)\right]$ & 15.849 & 14.434 & 9.718 & 0.482 & 0.213 & 0.356 \\
\hline \multicolumn{7}{|c|}{ (M2) Pareto $+t$ copula: positive dependence } \\
\hline $\mathbb{E}[\boldsymbol{X} \mid\{S=K\}]$ & 16.228 & 13.042 & 10.562 & 0.399 & 0.355 & 0.288 \\
\hline $\boldsymbol{K}_{\mathrm{M}}\left[\boldsymbol{X} ; \mathcal{K}_{d}(K)\right]$ & 17.689 & 12.481 & 9.830 & 0.759 & 0.663 & 0.475 \\
\hline \multicolumn{7}{|c|}{ (M3) Pareto $+t$ copula: no correlation } \\
\hline $\mathbb{E}[\boldsymbol{X} \mid\{S=K\}]$ & 17.479 & 11.368 & 10.562 & 0.517 & 0.530 & 0.288 \\
\hline $\boldsymbol{K}_{\mathrm{M}, 1}\left[\boldsymbol{X} ; \mathcal{K}_{d}(K)\right]$ & 25.678 & 3.107 & 11.215 & 1.185 & 0.278 & 1.205 \\
\hline $\boldsymbol{K}_{\mathrm{M}, 2}\left[\boldsymbol{X} ; \mathcal{K}_{d}(K)\right]$ & 2.639 & 35.275 & 2.086 & 0.973 & 1.306 & 0.424 \\
\hline \multicolumn{7}{|c|}{ (M4) Pareto $+t$ copula: negative dependence } \\
\hline $\mathbb{E}[\boldsymbol{X} \mid\{S=K\}]$ & 19.062 & 9.272 & 10.562 & 0.556 & 0.614 & 0.288 \\
\hline $\boldsymbol{K}_{\mathrm{M}, 1}\left[\boldsymbol{X} ; \mathcal{K}_{d}(K)\right]$ & 28.353 & 0.684 & 10.962 & 2.125 & 1.646 & 2.154 \\
\hline $\boldsymbol{K}_{\mathrm{M}, 2}\left[\boldsymbol{X} ; \mathcal{K}_{d}(K)\right]$ & 0.710 & 38.385 & 0.905 & 1.719 & 3.537 & 2.705 \\
\hline
\end{tabular}

significantly change the resulting allocation. On the other hand, for Model (M3) and (M4), the conditional distributions are multimodal, and thus a more careful decision making may be required.

To investigate the standard errors of the estimators, we compute the estimates of Euler allocation and (local) modes of $f_{\boldsymbol{X}^{\prime} \mid\{S=K\}} 100$ times for each model. For each repetition, we simulate samples from $\boldsymbol{X}$ so that there are 500 samples in the region $\mathcal{K}_{d}(K, \delta)$. The estimates and standard errors are computed based on the 100 replications and the results are summarized in Table 3. We can again see that for Models (M1) and (M2) the mode of $\boldsymbol{X}^{\prime} \mid\{S=K\}$ is unique and the two allocations are close. On the other hand, for Models (M3) and (M4) where the conditional distributions are multimodal, the standard errors of the (local) modes are higher than those of the Euler allocation.

In the end, we compute the multimodality-adjusted allocated capitals (14) for (M3) and (M4). In each case, the parameters are specified as $M=2, \mathcal{X}=\left\{\boldsymbol{K}_{1}, \boldsymbol{K}_{2}\right\}$ and $\boldsymbol{w}=\left(w_{1}, w_{2}\right)$ with $w_{m} \propto f_{\boldsymbol{X}}\left(\boldsymbol{K}_{m}\right)$, where $\boldsymbol{K}_{1}=\boldsymbol{K}_{\mathrm{M}, 1}\left[\boldsymbol{X} ; \mathcal{K}_{d}(K)\right]=(26.726,2.114,11.158)$ and $\boldsymbol{K}_{2}=\boldsymbol{K}_{\mathrm{M}, 2}\left[\boldsymbol{X} ; \mathcal{K}_{d}(K)\right]=(1.505,37.203,1.291)$ for (M3), and $\boldsymbol{K}_{1}=(28.589,0.432,10.978)$ and $\boldsymbol{K}_{2}=(0.326,39.314,0.358)$ for (M4). For both (M3) and (M4), the first and third units incur losses when $\left\{\boldsymbol{X}=\boldsymbol{K}_{1}\right\}$ occurs, and the second unit incurs a large loss when 
$\left\{\boldsymbol{X}=\boldsymbol{K}_{2}\right\}$ happens. The probability weights of the scenarios are given by $\boldsymbol{w}=(0.509,0.490)$ for (M3) and $\boldsymbol{w}=(0.272,0.727)$ for (M4). The two scenarios $\boldsymbol{K}_{1}$ and $\boldsymbol{K}_{2}$ are almost equally likely to occur for (M3), and the second scenario $\boldsymbol{K}_{2}$ is more likely to occur for (M4). Based on $\boldsymbol{w}$ and $\mathcal{X}$, the baseline allocations $\boldsymbol{K}_{\boldsymbol{w}, \mathcal{X}}$ are given by $(14.357,19.323,6.319)$ for $(\mathrm{M} 3)$ and $(8.038,28.705,3.256)$ for (M4). As seen in Table 3 , these allocations are not quite close to the Euler allocations since $\boldsymbol{K}_{\boldsymbol{w}, \mathcal{X}}$ is calculated based only on the two points $\boldsymbol{K}_{1}$ and $\boldsymbol{K}_{2}$ in $\mathcal{K}_{d}(K)$.

On computing the multimodality adjustments, we consider two cases when $\boldsymbol{K}_{\boldsymbol{w}, \mathcal{X}}$ or Euler allocations are used as baseline allocations. If $\boldsymbol{K}_{\boldsymbol{w}, \mathcal{X}}$ is the baseline allocation, the average loss $w_{1}\left(\boldsymbol{K}_{1}-\boldsymbol{K}_{\boldsymbol{w}, \mathcal{X}}\right)^{+}+w_{2}\left(\boldsymbol{K}_{2}-\right.$ $\left.\boldsymbol{K}_{\boldsymbol{w}, \mathcal{X}}\right)^{+}$in the multimodality adjustment is given by $(6.303,11.204,0.000)$ for (M3) and $(5.607,22.742,0.000)$ for (M4). If Euler allocation is used as a baseline allocation, the average loss is given by $(4.873,9.828,0.000)$ for (M3) and $(2.618,14.775,0.428)$ for (M4). In all cases, the average loss incurred in the second unit is larger than those in the first and third units since the second unit incurs a large loss when the second scenario $\left\{\boldsymbol{X}=\boldsymbol{K}_{2}\right\}$ occurs. Moreover, for (M4), the probability that the scenario $\left\{\boldsymbol{X}=\boldsymbol{K}_{2}\right\}$ occurs is higher than that of the first scenario $\left\{\boldsymbol{X}=\boldsymbol{K}_{1}\right\}$. Therefore, the analysis of the modes of $\boldsymbol{X} \mid\{S=K\}$ reveals that a large multimodality adjustment should be applied to $\boldsymbol{X}_{2}$ to increase the soundness of risk allocations.

\section{Conclusion}

Motivated from stress testing of risk allocations, we investigated properties of the conditional distribution of $\boldsymbol{X}$ given the sum constraint $\{S=K\}$, and also introduced the novel risk allocation method called maximum likelihood allocation (MLA). The superlevel set of $\boldsymbol{X} \mid\{S=K\}$ can be regarded as a set of stressed (severe and plausible) scenarios, and the modality of $\boldsymbol{X} \mid\{S=K\}$ can be interpreted as a variety of risky scenarios, which turned out to be an important feature in risk assessment related to the soundness of risk allocations. We then studied modality, dependence and tail behavior of $\boldsymbol{X} \mid\{S=K\}$, most of which are inherited from those of the unconditional loss $\boldsymbol{X}$. We then investigated how to incorporate the knowledge of the modality of $\boldsymbol{X} \mid\{S=K\}$ for more sound risk management. Under unimodality, we defined MLA as a mode of $\boldsymbol{X} \mid\{S=K\}$, and studied its properties as a risk allocation principle, such as translation invariance and positive homogeneity. Under multimodality, we considered the so-called multimodality adjustment to increase the soundness of risk allocations based on the multiple modes. Euler allocation and MLA were then compared in numerical experiments. Through the experiments, we demonstrated that $\boldsymbol{X} \mid\{S=$ $K\}$ is typically unimodal, and Euler allocation and MLA lead to close values when $\boldsymbol{X}$ possesses positive dependence. On the other hand, when the losses are negatively dependent, multimodality is likely to occur, and searching for the modes of $\boldsymbol{X} \mid\{S=K\}$ is beneficial for discovering hidden risky scenarios, evaluating the soundness of risk allocations, and eventually increasing the soundness of risk allocations by imposing the multimodality adjustment.

Although we empirically observed the relationship between multimodality of $\boldsymbol{X} \mid\{S=K\}$ and negative dependence of $\boldsymbol{X}$, this relationship requires further theoretical investigation. Another aspect of future research is to study more distributional properties, such as tail dependence and measures of concordance, of $\boldsymbol{X} \mid\{S=K\}$ especially without assuming the existence of a density. Unlike Euler allocations, estimation of MLAs is not a straightforward problem in general but various methods are known for estimating modes of 
multivariate distributions. For applying the MLA principle in practice, efficient estimation methods of the modes of multivariate distributions in high dimensions need to be explored further. An economic justification of the MLA principle is also an interesting direction for future research. In addition, an extension of the multimodality adjustment to general sets of scenarios is another avenue to explore since the multimodality adjustment proposed in this paper relies on the assumption that the set of scenarios is finite. In the end, efficient simulation approaches of $\boldsymbol{X} \mid\{S=K\}$ may need to rely on MCMC methods as introduced in Appendix F, and further investigation is required to assess how beneficial the distributional properties proven in this paper are to construct efficient MCMC methods since the performance of MCMC methods typically depends on tail-heaviness and modality of the target distribution.

\section{Funding}

This research was funded by NSERC through Discovery Grant RGPIN-5010-2015.

\section{Declaration of interests}

Declarations of interest: none.

\section{Acknowledgments}

We would like to thank the editor and three anonymous referees for their insightful comments on our manuscript.

\section{References}

Asimit, V., Peng, L., Wang, R., and Yu, A. (2019). An efficient approach to quantile capital allocation and sensitivity analysis. Mathematical Finance, 29(4):1131-1156.

Aumann, R. J. and Shapley, L. S. (2015). Values of non-atomic games. Princeton University Press, Princeton, New Jersey.

Balkema, G. and Nolde, N. (2010). Asymptotic independence for unimodal densities. Advances in Applied Probability, 42(2):411-432.

Breuer, T., Jandacka, M., Rheinberger, K., and Summer, M. (2018). How to find plausible, severe, and useful stress scenarios. Eighteenth issue (September 2009) of the International Journal of Central Banking.

Carreira-Perpinán, M. A. (2015). A review of mean-shift algorithms for clustering. arXiv preprint arXiv:1503.0068\%.

Chen, Y.-C., Genovese, C. R., Wasserman, L., et al. (2016). A comprehensive approach to mode clustering. Electronic Journal of Statistics, 10(1):210-241.

Denault, M. (2001). Coherent allocation of risk capital. Journal of Risk, 4(1):1-34. 
Dhaene, J., Tsanakas, A., Valdez, E. A., and Vanduffel, S. (2012). Optimal capital allocation principles. Journal of Risk and Insurance, 79(1):1-28.

Dharmadhikari, S. and Joag-Dev, K. (1988). Unimodality, convexity, and applications. Elsevier.

Ding, P. (2016). On the conditional distribution of the multivariate t distribution. The American Statistician, 70(3):293-295.

Fang, K. W. (2018). Symmetric multivariate and related distributions. Chapman and Hall/CRC.

Fernandez, C., Osiewalski, J., and Steel, M. F. (1995). Modeling and inference with $v$-spherical distributions. Journal of the American Statistical Association, 90(432):1331-1340.

Fernández, C. and Steel, M. F. (1998). On bayesian modeling of fat tails and skewness. Journal of the American Statistical Association, 93(441):359-371.

Fukunaga, K. and Hostetler, L. (1975). The estimation of the gradient of a density function, with applications in pattern recognition. IEEE Transactions on information theory, 21(1):32-40.

Furman, E. and Landsman, Z. (2006). Tail variance premium with applications for elliptical portfolio of risks. ASTIN Bulletin: The Journal of the IAA, 36(2):433-462.

Furman, E., Wang, R., and Zitikis, R. (2017). Gini-type measures of risk and variability: Gini shortfall, capital allocations, and heavy-tailed risks. Journal of Banking \& Finance, 83:70-84.

Huang, J.-J., Lee, K.-J., Liang, H., and Lin, W.-F. (2009). Estimating value at risk of portfolio by conditional copula-garch method. Insurance: Mathematics and economics, 45(3):315-324.

Joe, H. and Li, H. (2019). Tail densities of skew-elliptical distributions. Journal of Multivariate Analysis, 171:421-435.

Jondeau, E. and Rockinger, M. (2006). The copula-garch model of conditional dependencies: An international stock market application. Journal of international money and finance, 25(5):827-853.

Kalkbrener, M. (2005). An axiomatic approach to capital allocation. Mathematical Finance, 15(3):425-437.

Karlin, S. and Rinott, Y. (1980a). Classes of orderings of measures and related correlation inequalities. i. multivariate totally positive distributions. Journal of Multivariate Analysis, 10(4):467-498.

Karlin, S. and Rinott, Y. (1980b). Classes of orderings of measures and related correlation inequalities ii. multivariate reverse rule distributions. Journal of Multivariate Analysis, 10(4):499-516.

Koike, T. and Hofert, M. (2020). Markov chain monte carlo methods for estimating systemic risk allocations. Risks, 8(1):6.

Koike, T. and Minami, M. (2019). Estimation of risk contributions with mcmc. Quantitative Finance, 19(9):1579-1597.

Laeven, R. J. and Goovaerts, M. J. (2004). An optimization approach to the dynamic allocation of economic capital. Insurance: Mathematics and Economics, 35(2):299-319. 
Li, H. (2013). Toward a copula theory for multivariate regular variation. In Copulae in mathematical and quantitative finance, pages 177-199. Springer.

Maume-Deschamps, V., Rullière, D., and Said, K. (2016). On a capital allocation by minimization of some risk indicators. European Actuarial Journal, 6(1):177-196.

McNeil, A. J., Frey, R., and Embrechts, P. (2015). Quantitative risk management: Concepts, techniques and tools. Princeton University Press, Princeton.

Müller, A. and Stoyan, D. (2002). Comparison methods for stochastic models and risks, volume 389. Wiley New York.

Norkin, V. and Roenko, N. (1991). $\alpha$-concave functions and measures and their applications. Cybernetics and Systems Analysis, 27(6):860-869.

Osiewalski, J. (1993). Robust bayesian inference in lq-spherical models. Biometrika, 80(2):456-460.

Resnick, S. I. (2007). Heavy-tail phenomena: probabilistic and statistical modeling. Springer Science \& Business Media.

Roth, M. (2012). On the multivariate $t$ distribution. Linköping University Electronic Press.

Saumard, A. and Wellner, J. A. (2014). Log-concavity and strong log-concavity: a review. Statistics Surveys, $8: 45$.

Schmidt, R. (2002). Tail dependence for elliptically contoured distributions. Mathematical Methods of Operations Research, 55(2):301-327.

Shaked, M. and Shanthikumar, J. G. (2007). Stochastic orders. Springer Science \& Business Media.

Sweeting, T. J. et al. (1986). On a converse to scheffé's theorem. The Annals of Statistics, 14(3):1252-1256.

Tasche, D. (1995). Risk contributions and performance measurement. Working Paper, Techische Universität München.

Tasche, D. (2001). Conditional expectation as quantile derivative. arXiv preprint math/0104190.

Wang, B. and Wang, R. (2011). The complete mixability and convex minimization problems with monotone marginal densities. Journal of Multivariate Analysis, 102(10):1344-1360.

\section{Appendices}

\section{A Proofs}

\section{Proof of Proposition 1}

Proof. Notice that $\left(\boldsymbol{X}^{\prime}, S\right)=A \boldsymbol{X} \sim \mathcal{E}_{d}\left(A \boldsymbol{\mu}, A \Sigma A^{\top}, \psi\right)$ where $A=\left(\begin{array}{cc}\boldsymbol{I}_{d} & \mathbf{0}_{d} \\ \mathbf{1}_{d}^{\top} & 1\end{array}\right) \in \mathbb{R}^{d \times d}$. Therefore, the conditional distribution $\boldsymbol{X}^{\prime} \mid\{S=K\}$ also follows an elliptical distribution with the location parameter $\boldsymbol{\mu}_{K}$ 
and the dispersion parameter $\Sigma_{K}$ as specified in (7). The corresponding characteristic generator $\psi_{K}$ can be specified through Theorem 2.18 of Fang (2018). If $\boldsymbol{X}$ admits a density with density generator $g$, then

$$
f_{\boldsymbol{X}^{\prime} \mid\{S=K\}}\left(\boldsymbol{x}^{\prime}\right)=\frac{f_{\left(\boldsymbol{X}^{\prime}, S\right)}\left(\boldsymbol{x}^{\prime}, K\right)}{f_{S}(K)} \propto g_{d}\left(\frac{1}{2}\left(\boldsymbol{x}^{\prime}-\boldsymbol{\mu}^{\prime}, K-\mu_{S}\right)^{\top}\left(\begin{array}{cc}
\Sigma^{\prime} & \left(\Sigma \mathbf{1}_{d}\right)^{\prime} \\
\left(\Sigma \mathbf{1}_{d}\right)^{\prime \top} & \sigma_{S}^{2}
\end{array}\right)^{-1}\left(\boldsymbol{x}^{\prime}-\boldsymbol{\mu}^{\prime}, K-\mu_{S}\right)\right) .
$$

The quadratic term reduces to

$$
\left(\boldsymbol{x}^{\prime}-\boldsymbol{\mu}^{\prime}, K-\mu_{S}\right)^{\top}\left(\begin{array}{cc}
\Sigma^{\prime} & \left(\Sigma \mathbf{1}_{d}\right)^{\prime} \\
\left(\Sigma \mathbf{1}_{d}\right)^{\prime \top} & \sigma_{S}^{2}
\end{array}\right)^{-1}\left(\boldsymbol{x}^{\prime}-\boldsymbol{\mu}^{\prime}, K-\mu_{S}\right)=\left(\boldsymbol{x}^{\prime}-\boldsymbol{\mu}_{K}\right)^{\top} \Sigma_{K}^{-1}\left(\boldsymbol{x}^{\prime}-\boldsymbol{\mu}_{K}\right)+\left(\frac{K-\mu_{S}}{\sigma_{S}}\right)^{2} .
$$

Therefore, we have that

$$
f_{\boldsymbol{X}^{\prime} \mid\{S=K\}}\left(\boldsymbol{x}^{\prime}\right) \propto g\left(\frac{1}{2}\left(\boldsymbol{x}^{\prime}-\boldsymbol{\mu}_{K}\right)^{\top} \Sigma_{K}^{-1}\left(\boldsymbol{x}^{\prime}-\boldsymbol{\mu}_{K}\right)+\Delta_{K}\right)=g_{K}\left(\frac{1}{2}\left(\boldsymbol{x}^{\prime}-\boldsymbol{\mu}_{K}\right)^{\top} \Sigma_{K}^{-1}\left(\boldsymbol{x}^{\prime}-\boldsymbol{\mu}_{K}\right)\right),
$$

where $\Delta_{K}=\left(K-\mu_{S}\right)^{2} /\left(2 \sigma_{S}^{2}\right)$ and $g_{K}(t)=g\left(t+\Delta_{K}\right)$ as specified in (8).

\section{Proof of Proposition 2}

Proof. 1. By Proposition $1, \boldsymbol{X}^{\prime} \mid\{S=K\}$ follows a $d^{\prime}$-dimensional elliptical distribution with location vector $\boldsymbol{\mu}_{K}$, dispersion matrix $\Sigma_{K}$ and density generator $g_{K}$. Furthermore, $g_{K}$ is decreasing if $g$ is. Therefore, for $0<s \leq c_{K} t_{K}^{*} / \sqrt{\left|\Sigma_{K}\right|}$,

$$
\begin{aligned}
L_{s}\left(\boldsymbol{X}^{\prime} \mid\{S=K\}\right) & =\left\{\boldsymbol{x}^{\prime} \in \mathbb{R}^{d^{\prime}}: g_{K}\left(\frac{1}{2}\left(\boldsymbol{x}^{\prime}-\boldsymbol{\mu}_{K}\right)^{\top} \Sigma_{K}^{-1}\left(\boldsymbol{x}^{\prime}-\boldsymbol{\mu}_{K}\right)\right) \geq \frac{s \sqrt{\left|\Sigma_{K}\right|}}{c_{K}}\right\} \\
& =\left\{\boldsymbol{x}^{\prime} \in \mathbb{R}^{d^{\prime}}: 0 \leq\left(\boldsymbol{x}^{\prime}-\boldsymbol{\mu}_{K}\right)^{\top} \Sigma_{K}^{-1}\left(\boldsymbol{x}^{\prime}-\boldsymbol{\mu}_{K}\right) \leq 2\left\{g^{-1}\left(\frac{s \sqrt{\left|\Sigma_{K}\right|}}{c_{K}}\right)-\Delta_{K}\right\}\right\},
\end{aligned}
$$

which is a convex set with ellipsoid as surface. Moreover, when $s^{*}=c_{K} t_{K}^{*} / \sqrt{\left|\Sigma_{K}\right|}$, we have

$$
L_{s^{*}}\left(\boldsymbol{X}^{\prime} \mid\{S=K\}\right)=\left\{\boldsymbol{x}^{\prime} \in \mathbb{R}^{d^{\prime}}:\left(\boldsymbol{x}^{\prime}-\boldsymbol{\mu}_{K}\right)^{\top} \Sigma_{K}^{-1}\left(\boldsymbol{x}^{\prime}-\boldsymbol{\mu}_{K}\right)=0\right\}=\left\{\boldsymbol{\mu}_{K}\right\}
$$

and thus $\boldsymbol{X}^{\prime} \mid\{S=K\}$ has a mode $\boldsymbol{\mu}_{K}$.

2. For $t>0$ and $\boldsymbol{x}^{\prime} \in \mathbb{R}^{d^{\prime}}$, we have the equivalence relation:

$$
\boldsymbol{x}^{\prime} \in L_{t}\left(\boldsymbol{X}^{\prime} \mid\{S=K\}\right) \quad \text { if and only if } \quad\left(\boldsymbol{x}^{\prime}, K-\mathbf{1}_{d^{\prime}}^{\top} \boldsymbol{x}^{\prime}\right) \in L_{t f_{S}(K)}(\boldsymbol{X})
$$

since $f_{\boldsymbol{X}^{\prime} \mid\{S=K\}}\left(\boldsymbol{x}^{\prime}\right)=f_{\boldsymbol{X}}\left(\boldsymbol{x}^{\prime}, K-\mathbf{1}_{d^{\prime}}^{\top} \boldsymbol{x}^{\prime}\right) / f_{S}(K)$ and thus

$$
L_{t}\left(\boldsymbol{X}^{\prime} \mid\{S=K\}\right)=\left\{\boldsymbol{x}^{\prime} \in \mathbb{R}^{d^{\prime}}: f_{\boldsymbol{X}^{\prime} \mid\{S=K\}}\left(\boldsymbol{x}^{\prime}\right) \geq t\right\}=\left\{\boldsymbol{x}^{\prime} \in \mathbb{R}^{d^{\prime}}: f_{\boldsymbol{X}}\left(\boldsymbol{x}^{\prime}, K-\mathbf{1}_{d^{\prime}}^{\top} \boldsymbol{x}^{\prime}\right) \geq t f_{S}(K)\right\} .
$$

Suppose $\boldsymbol{x}^{\prime}, \boldsymbol{y}^{\prime} \in L_{t}\left(\boldsymbol{X}^{\prime} \mid\{S=K\}\right)$. By (18), we have that $\left(\boldsymbol{x}^{\prime}, K-\mathbf{1}_{d^{\prime}}^{\top} \boldsymbol{x}^{\prime}\right), \quad\left(\boldsymbol{y}^{\prime}, K-\mathbf{1}_{d^{\prime}}^{\top} \boldsymbol{y}^{\prime}\right) \in$ $L_{t f_{S}(K)}(\boldsymbol{X})$. Since $\boldsymbol{X}$ is convex unimodal, $L_{t f_{S}(K)}(\boldsymbol{X})$ is a convex set. Therefore, we have, for $\theta \in(0,1)$, that

$$
\begin{aligned}
\theta\left(\boldsymbol{x}^{\prime}, K-\mathbf{1}_{d^{\prime}} \boldsymbol{x}^{\prime}\right)+(1-\theta)\left(\boldsymbol{y}^{\prime}, K-\mathbf{1}_{d^{\prime}}^{\top} \boldsymbol{y}^{\prime}\right) & =\left(\theta \boldsymbol{x}^{\prime}+(1-\theta) \boldsymbol{y}^{\prime}, \theta\left(K-\mathbf{1}_{d^{\prime}}^{\top} \boldsymbol{x}^{\prime}\right)+(1-\theta)\left(K-\mathbf{1}_{d^{\prime}}^{\top} \boldsymbol{y}^{\prime}\right)\right. \\
& =\left(\theta \boldsymbol{x}^{\prime}+(1-\theta) \boldsymbol{y}^{\prime}, K-\mathbf{1}_{d^{\prime}}^{\top}\left(\theta \boldsymbol{x}^{\prime}+(1-\theta) \boldsymbol{y}^{\prime}\right)\right) \in L_{t f_{S}(K)}(\boldsymbol{X}),
\end{aligned}
$$

which implies that $\theta \boldsymbol{x}^{\prime}+(1-\theta) \boldsymbol{y}^{\prime} \in L_{t}\left(\boldsymbol{X}^{\prime} \mid S=K\right)$ by (18). 


\section{Proof of Proposition 3}

Proof. Let $\tilde{\boldsymbol{X}}=\left(\boldsymbol{X}^{\prime}, K-X_{d}\right)$. Since the density of $\tilde{\boldsymbol{X}}$ is written as $f_{\tilde{\boldsymbol{X}}}\left(x_{1}, \ldots, x_{d}\right)=f_{\boldsymbol{X}}\left(x_{1}, \ldots, x_{d^{\prime}}, K-x_{d}\right)$, we have, by (5), that

$$
f_{\boldsymbol{X}^{\prime} \mid\{S=K\}}\left(\boldsymbol{x}^{\prime}\right)=\frac{f_{\boldsymbol{X}}\left(\boldsymbol{x}^{\prime}, K-\mathbf{1}_{d^{\prime}}^{\top} \boldsymbol{x}^{\prime}\right)}{f_{S}(K)}=\frac{f_{\tilde{\boldsymbol{X}}}\left(\boldsymbol{x}^{\prime}, \mathbf{1}_{d^{\prime}}^{\top} \boldsymbol{x}^{\prime}\right)}{f_{S}(K)}, \quad \boldsymbol{x}^{\prime} \in \mathbb{R}_{+}^{d^{\prime}} .
$$

Therefore, if $\tilde{\boldsymbol{X}}$ has a limit function $\tilde{\lambda}$, then the density of $\boldsymbol{X}^{\prime} \mid\{S=K\}$ satisfies

$$
\lim _{t \rightarrow \infty} \frac{f_{\boldsymbol{X}^{\prime} \mid\{S=K\}}\left(t \boldsymbol{y}^{\prime}\right)}{f_{\boldsymbol{X}^{\prime} \mid\{S=K\}}\left(t \boldsymbol{x}^{\prime}\right)}=\lim _{t \rightarrow \infty} \frac{f_{\tilde{\boldsymbol{X}}}\left(t \boldsymbol{y}^{\prime}, t \mathbf{1}_{d^{\prime}}^{\top} \boldsymbol{y}^{\prime}\right)}{f_{\tilde{\boldsymbol{X}}}\left(t \boldsymbol{x}^{\prime}, t \mathbf{1}_{d^{\prime}}^{\top} \boldsymbol{x}^{\prime}\right)}=\tilde{\lambda}\left(\left(\boldsymbol{x}^{\prime}, \mathbf{1}_{d^{\prime}}^{\top} \boldsymbol{x}^{\prime}\right),\left(\boldsymbol{y}^{\prime}, \mathbf{1}_{d^{\prime}}^{\top} \boldsymbol{y}^{\prime}\right)\right)=\lambda^{\prime}\left(\boldsymbol{x}^{\prime}, \boldsymbol{y}^{\prime}\right),
$$

for any $\boldsymbol{x}^{\prime}, \boldsymbol{y}^{\prime} \in \mathbb{R}_{+}^{d^{\prime}}$ since $\left(\boldsymbol{x}^{\prime}, \mathbf{1}_{d^{\prime}}^{\top} \boldsymbol{x}^{\prime}\right),\left(\boldsymbol{y}^{\prime}, \mathbf{1}_{d^{\prime}}^{\top} \boldsymbol{y}^{\prime}\right) \in \mathbb{R}_{+}^{d}$. Similarly, if $\tilde{\boldsymbol{X}}$ is $\operatorname{MRV}(\infty)$, then

$$
\lim _{t \rightarrow \infty} \frac{f_{\boldsymbol{X}^{\prime} \mid\{S=K\}}\left(s t \boldsymbol{x}^{\prime}\right)}{f_{\boldsymbol{X}^{\prime} \mid\{S=K\}}\left(t \boldsymbol{x}^{\prime}\right)}=\lim _{t \rightarrow \infty} \frac{f_{\tilde{\boldsymbol{X}}}\left(s t \boldsymbol{x}^{\prime}, s t \mathbf{1}_{d^{\prime}}^{\top} \boldsymbol{x}^{\prime}\right)}{f_{\tilde{\boldsymbol{X}}}\left(t \boldsymbol{x}^{\prime}, t \mathbf{1}_{d^{\prime}}^{\top} \boldsymbol{x}^{\prime}\right)}= \begin{cases}0, & s>1, \\ \infty, & 0<s<1,\end{cases}
$$

for any $s>0$ and $\boldsymbol{x}^{\prime} \in \mathbb{R}_{+}^{d^{\prime}}$.

\section{Proof of Proposition 4}

Proof. Proposition 1 yields that $\boldsymbol{X}^{\prime} \mid\{S=K\}$ follows a $d^{\prime}$-dimensional elliptical distribution with location vector $\boldsymbol{\mu}_{K}$, dispersion matrix $\Sigma_{K}$ and density generator $g_{K}$. If $g$ is regularly varying, then

$$
\begin{aligned}
\lim _{t \rightarrow \infty} \frac{f_{\boldsymbol{X}^{\prime} \mid\{S=K\}}\left(t \boldsymbol{y}^{\prime}\right)}{f_{\boldsymbol{X}^{\prime} \mid\{S=K\}}\left(t \boldsymbol{x}^{\prime}\right)} & =\lim _{t \rightarrow \infty} \frac{g_{K}\left(\frac{1}{2}\left(t \boldsymbol{y}^{\prime}-\boldsymbol{\mu}_{K}\right)^{\top} \Sigma_{K}^{-1}\left(t \boldsymbol{y}^{\prime}-\boldsymbol{\mu}_{K}\right)\right)}{g_{K}\left(\frac{1}{2}\left(t \boldsymbol{x}^{\prime}-\boldsymbol{\mu}_{K}\right)^{\top} \Sigma_{K}^{-1}\left(t \boldsymbol{x}^{\prime}-\boldsymbol{\mu}_{K}\right)\right)} \\
& =\lim _{t \rightarrow \infty} \frac{g\left(\frac{1}{2} t^{2}\left(\boldsymbol{y}^{\prime}-\boldsymbol{\mu}_{K} / t\right)^{\top} \Sigma_{K}^{-1}\left(\boldsymbol{y}^{\prime}-\boldsymbol{\mu}_{K} / t\right)+\Delta_{K}\right)}{g\left(\frac{1}{2} t^{2}\left(\boldsymbol{x}^{\prime}-\boldsymbol{\mu}_{K} / t\right)^{\top} \Sigma_{K}^{-1}\left(\boldsymbol{x}^{\prime}-\boldsymbol{\mu}_{K} / t\right)+\Delta_{K}\right)} \\
& =\lim _{t \rightarrow \infty} \frac{g\left(\frac{1}{2} t^{2} \boldsymbol{y}^{\prime \top} \Sigma_{K}^{-1} \boldsymbol{y}^{\prime}\right)}{g\left(\frac{1}{2} t^{2} \boldsymbol{x}^{\prime \top} \Sigma_{K}^{-1} \boldsymbol{x}^{\prime}\right)}=\lambda_{g}\left(\boldsymbol{x}^{\prime \top} \Sigma_{K}^{-1} \boldsymbol{x}^{\prime}, \boldsymbol{y}^{\prime \top} \Sigma_{K}^{-1} \boldsymbol{y}^{\prime}\right)=\lambda_{K}\left(\boldsymbol{x}^{\prime}, \boldsymbol{y}^{\prime}\right),
\end{aligned}
$$

for any $\boldsymbol{x}^{\prime}, \boldsymbol{y}^{\prime} \in \mathbb{R}^{d^{\prime}}$, where the third equality comes from continuity of $g$ and the fourth equality holds since $\boldsymbol{x}^{\prime \top} \Sigma_{K}^{-1} \boldsymbol{x}^{\prime}, \boldsymbol{y}^{\prime \top} \Sigma_{K}^{-1} \boldsymbol{y}^{\prime}>0$. Therefore, $\boldsymbol{X}^{\prime} \mid\{S=K\}$ is $\operatorname{MRV}\left(\lambda_{K}\right)$. For the rapidly varying case,

$$
\lim _{t \rightarrow \infty} \frac{f_{\boldsymbol{X}^{\prime} \mid\{S=K\}}\left(s t \boldsymbol{x}^{\prime}\right)}{f_{\boldsymbol{X}^{\prime} \mid\{S=K\}}\left(t \boldsymbol{x}^{\prime}\right)}=\lim _{t \rightarrow \infty} \frac{g\left(\frac{1}{2} t^{2} s^{2} \boldsymbol{x}^{\prime \top} \Sigma_{K}^{-1} \boldsymbol{x}^{\prime}\right)}{g\left(\frac{1}{2} t^{2} \boldsymbol{x}^{\prime \top} \Sigma_{K}^{-1} \boldsymbol{x}^{\prime}\right)}= \begin{cases}0, & s>1, \\ \infty, & 0<s<1,\end{cases}
$$

for any $s>0$ and $\boldsymbol{x}^{\prime}, \boldsymbol{y}^{\prime} \in \mathbb{R}^{d^{\prime}}$ since $s>1$ if and only if $s^{2}>1$ and $0<s<1$ if and only if $0<s^{2}<1$ for $s>0$. Therefore, $\boldsymbol{X}^{\prime} \mid\{S=K\}$ is rapidly varying.

\section{Proof of Proposition 5}

Proof. 1. Translation invariance: Let $\tilde{\boldsymbol{X}}=\boldsymbol{X}+\boldsymbol{c}, \tilde{S}=S+\mathbf{1}_{d}^{\top} \boldsymbol{c}$ and $\tilde{K}=K+\mathbf{1}_{d}^{\top} \boldsymbol{c}$. Since $f_{\boldsymbol{X}+\boldsymbol{c}}(\boldsymbol{x})=$ $f_{\boldsymbol{X}}(\boldsymbol{x}-\boldsymbol{c})$, we have that

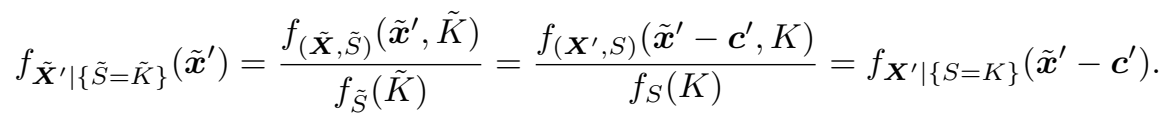

Therefore, uniqueness of the maximizer of $f_{\boldsymbol{X}^{\prime} \mid\{S=K\}}$ implies that of $f_{\tilde{\boldsymbol{X}}^{\prime} \mid\{\tilde{S}=\tilde{K}\}}$, and these maximizers are related via $\boldsymbol{K}_{\mathrm{M}}\left[\boldsymbol{X}+\boldsymbol{c} ; \mathcal{K}_{d}\left(K+\mathbf{1}_{d}^{\top} \boldsymbol{c}\right)\right]=\boldsymbol{K}_{\mathrm{M}}\left[\boldsymbol{X} ; \mathcal{K}_{d}(K)\right]+\boldsymbol{c}$. 
2. Positive homogeneity: Let $\tilde{\boldsymbol{X}}=c \boldsymbol{X}, \tilde{S}=c S$ and $\tilde{K}=c K$. Since $f_{c \boldsymbol{X}}(\boldsymbol{x})=f_{\boldsymbol{X}}(\boldsymbol{x} / c)$, we have that

$$
f_{\tilde{\boldsymbol{X}}^{\prime} \mid\{\tilde{S}=\tilde{K}\}}\left(\tilde{\boldsymbol{x}}^{\prime}\right)=\frac{f_{(\tilde{\boldsymbol{X}}, \tilde{S})}\left(\tilde{\boldsymbol{x}}^{\prime}, \tilde{K}\right)}{f_{\tilde{S}}(\tilde{K})}=\frac{f_{\left(\boldsymbol{X}^{\prime}, S\right)}\left(\tilde{\boldsymbol{x}}^{\prime} / c, K\right)}{f_{S}(K)}=f_{\boldsymbol{X}^{\prime} \mid\{S=K\}}\left(\tilde{\boldsymbol{x}}^{\prime} / c\right) .
$$

As seen in the case of translation invariance, this equality implies that $\tilde{\boldsymbol{X}} \in \mathcal{U}_{d}(\tilde{K})$ and $\boldsymbol{K}_{\mathrm{M}}\left[\boldsymbol{X} ; \mathcal{K}_{d}(c K)\right]=$ $c \boldsymbol{K}_{\mathrm{M}}\left[\boldsymbol{X} ; \mathcal{K}_{d}(K)\right]$

3. Symmetry: Without loss of generality, consider $i=1$ and $j=2$. Let $\tilde{\boldsymbol{X}}=\left(X_{2}, X_{1}, \boldsymbol{X}_{-(1,2)}\right)$ and $\tilde{S}=\mathbf{1}_{d}^{\top} \tilde{\boldsymbol{X}}$, where $\boldsymbol{x}_{-(1,2)}$ is a shorthand for $\left(x_{3}, \ldots, x_{d}\right)$ for $\boldsymbol{x} \in \mathbb{R}^{d}$. Then $f_{\tilde{\boldsymbol{X}}}(\boldsymbol{x})=f_{\boldsymbol{X}}(\tilde{\boldsymbol{x}})$ for $\boldsymbol{x}=\left(x_{1}, x_{2}, \boldsymbol{x}_{-(1,2)}\right) \in \mathbb{R}^{d}$ and $\tilde{\boldsymbol{x}}=\left(x_{2}, x_{1}, \boldsymbol{x}_{-(1,2)}\right) \in \mathbb{R}^{d}$. Moreover, when $\boldsymbol{X} \stackrel{\mathrm{d}}{=} \tilde{\boldsymbol{X}}$, we have that $\tilde{\boldsymbol{X}} \in \mathcal{U}_{d}(K)$ and $f_{\boldsymbol{X}}=f_{\tilde{\boldsymbol{X}}}$. Consequently, we have that

$$
f_{\boldsymbol{X}^{\prime} \mid\{S=K\}}\left(\boldsymbol{x}^{\prime}\right)=\frac{f_{\boldsymbol{X}}\left(\boldsymbol{x}^{\prime}, K-\mathbf{1}_{d^{\prime}}^{\top} \boldsymbol{x}^{\prime}\right)}{f_{S}(K)}=\frac{f_{\tilde{\boldsymbol{X}}}\left(\boldsymbol{x}^{\prime}, K-\mathbf{1}_{d^{\prime}}^{\top} \boldsymbol{x}^{\prime}\right)}{f_{S}(K)}=\frac{f_{\boldsymbol{X}}\left(\tilde{\boldsymbol{x}}^{\prime}, K-\mathbf{1}_{d^{\prime}}^{\top} \tilde{\boldsymbol{x}}^{\prime}\right)}{f_{S}(K)}=f_{\boldsymbol{X}^{\prime} \mid\{S=K\}}\left(\tilde{\boldsymbol{x}}^{\prime}\right),
$$

where the third equation holds since $\mathbf{1}_{d^{\prime}}^{\top} \boldsymbol{x}^{\prime}=\mathbf{1}_{d^{\prime}}^{\top} \tilde{\boldsymbol{x}}^{\prime}$. Now suppose that $\boldsymbol{K}_{\mathrm{M}}\left[\boldsymbol{X} ; \mathcal{K}_{d}(K)\right]_{1} \neq \boldsymbol{K}_{\mathrm{M}}\left[\boldsymbol{X} ; \mathcal{K}_{d}(K)\right]_{2}$. Then two different vectors $\boldsymbol{K}_{\mathrm{M}}\left[\boldsymbol{X} ; \mathcal{K}_{d}(K)\right]$ and $\left(\boldsymbol{K}_{\mathrm{M}}\left[\boldsymbol{X} ; \mathcal{K}_{d}(K)\right]_{2}, \boldsymbol{K}_{\mathrm{M}}\left[\boldsymbol{X} ; \mathcal{K}_{d}(K)\right]_{1}, \boldsymbol{K}_{\mathrm{M}}\left[\boldsymbol{X} ; \mathcal{K}_{d}(K)\right]_{-(1,2)}\right)$ attain the maximum of $f_{\boldsymbol{X}^{\prime} \mid\{S=K\}}$ by (19). Since $\boldsymbol{K}_{\mathrm{M}}\left[\boldsymbol{X} ; \mathcal{K}_{d}(K)\right]$ is obtained by the unique maximizer of $f_{\boldsymbol{X}^{\prime} \mid\{S=K\}}\left(\boldsymbol{x}^{\prime}\right)$, this leads to a contradiction.

4. Continuity: When $f_{n}$ is uniformly continuous and bounded for $n=1,2, \ldots$, the sequence $\left(f_{n}\right)$ is asymptotically uniformly equicontinuous and bounded in the sense introduced in Sweeting et al. (1986). Together with the assumption that $\boldsymbol{X}_{n} \rightarrow \boldsymbol{X}$ weakly, Theorem 2 of Sweeting et al. (1986) implies that $f_{n} \rightarrow f$ pointwise and uniformly in $\mathbb{R}^{d}$ for the uniformly continuous density $f$ of $\boldsymbol{X}$. Define $g_{n}\left(\boldsymbol{x}^{\prime}\right)=f_{n}\left(\boldsymbol{x}^{\prime}, K-\mathbf{1}_{d^{\prime}}^{\top} \boldsymbol{x}^{\prime}\right)$ for $n=1,2, \ldots$ and $g\left(\boldsymbol{x}^{\prime}\right)=f\left(\boldsymbol{x}^{\prime}, K-\mathbf{1}_{d^{\prime}}^{\top} \boldsymbol{x}^{\prime}\right), \boldsymbol{x}^{\prime} \in \mathbb{R}^{d^{\prime}}$. By (5) and since $\boldsymbol{X}_{n}, \boldsymbol{X} \in \mathcal{U}_{d}(K)$, the maximizers of $g_{n}$ and $g$ are uniquely determined. Denote them as $\boldsymbol{x}_{n}^{*}=\underset{\boldsymbol{x} \in \mathbb{R}^{d^{\prime}}}{\operatorname{argmax}} g_{n}(\boldsymbol{x})$ and $\boldsymbol{x}^{*}=\underset{\boldsymbol{x} \in \mathbb{R}^{d^{\prime}}}{\operatorname{argmax}} g(\boldsymbol{x})$. By definition of $\boldsymbol{x}_{n}^{*}$, we have that

$$
g_{n}\left(\boldsymbol{x}_{n}^{*}\right) \geq g_{n}(\boldsymbol{x}) \text { for any } \quad \boldsymbol{x} \in \mathbb{R}^{d^{\prime}}
$$

Since $g_{n}$ converges uniformly to $g$, it holds that

$$
g\left(\limsup _{n \rightarrow \infty} \boldsymbol{x}_{n}^{*}\right) \geq g(\boldsymbol{x}) \quad \text { and } \quad g\left(\liminf _{n \rightarrow \infty} \boldsymbol{x}_{n}^{*}\right) \geq g(\boldsymbol{x}) \quad \text { for any } \quad \boldsymbol{x} \in \mathbb{R}^{d^{\prime}} .
$$

If $\lim \sup _{n \rightarrow \infty} \boldsymbol{x}_{n}^{*}>\liminf _{n \rightarrow \infty} \boldsymbol{x}_{n}^{*}$, then two points attain the maximum of $g$, which contradicts the uniqueness of the maximizer of $g$. As a consequence, $\limsup _{n \rightarrow \infty} \boldsymbol{x}_{n}^{*}=\liminf _{n \rightarrow \infty} \boldsymbol{x}_{n}^{*}=\lim _{n \rightarrow \infty} \boldsymbol{x}_{n}^{*}=$ $\boldsymbol{x}^{*}$ and thus $\lim _{n \rightarrow \infty} \boldsymbol{K}_{\mathrm{M}}\left[\boldsymbol{X}_{n} ; \mathcal{K}_{d}(K)\right]=\boldsymbol{K}_{\mathrm{M}}\left[\boldsymbol{X} ; \mathcal{K}_{d}(K)\right]$.

\section{B Modality and $s$-concave densities}

As we saw in Section 3.3, neither joint unimodality nor marginal unimodality implies the other . However, unimodality is preserved under marginalization for some specific class of densities, so-called $s$-concave densities. In this appendix we briefly introduce the connection between unimodality and $s$-concavity of the conditional distribution given a constant sum. 
Definition 6 (s-concavity). For $s \in \mathbb{R}$, a density $f$ on $\mathbb{R}^{d}$ is called $s$-concave on a convex set $A \subseteq \mathbb{R}^{d}$ if

$$
f(\theta \boldsymbol{x}+(1-\theta) \boldsymbol{y}) \geq M_{s}(f(\boldsymbol{x}), f(\boldsymbol{y}) ; \theta), \quad \boldsymbol{x}, \boldsymbol{y} \in A, \quad \theta \in(0,1),
$$

where $M_{s}$ is called the generalized mean defined, by continuity, as

$$
M_{s}(a, b ; \theta)= \begin{cases}\left\{\theta a^{s}+(1-\theta) b^{s}\right\}^{1 / s}, & 0<s<\infty \text { or }(-\infty<s<0 \text { and } a b \neq 0), \\ 0, & -\infty<s<0 \text { and } a b=0, \\ a^{\theta} b^{1-\theta}, & s=0, \\ a \wedge b, & s=-\infty, \\ a \vee b, & s=+\infty,\end{cases}
$$

for $s \in \mathbb{R}, a, b \geq 0$ and $\theta \in(0,1)$.

Definition 6 of $s$-concavity is based on densities and can be extended to a measure-based definition for distributions that do not admit densities; see Dharmadhikari and Joag-Dev (1988). For $s=-\infty, s$ concavity is also known as quasi-concavity and 0-concavity is also known as log-concavity. By definition, for $0<s<\infty, f$ is $s$-concave if and only if $f^{s}$ is a concave function. As shown in Dharmadhikari and Joag-Dev (1988), the function $s \mapsto M_{s}(a, b ; \theta)$ is increasing for fixed $(a, b ; \theta)$. From this we have that $t$-concavity of $f$ implies $s$-concavity for $s<t$. Examples of $s$-concave densities include the skew-normal distributions (Balkema and Nolde, 2010), Wishart distributions, Dirichlet distributions with certain range of parameters (Dharmadhikari and Joag-Dev, 1988) and uniform distributions on a convex set in $\mathbb{R}^{d}$ (Norkin and Roenko, 1991).

Convex unimodality (Definition 1 ) is related to $s$-concavity since a density $f$ is convex unimodal if and only if it is $-\infty$-concave (Dharmadhikari and Joag-Dev, 1988). Therefore, $f$ is convex unimodal if it is $s$-concave for some $s \in \mathbb{R}$. Furthermore, it is straightforward to show that $\boldsymbol{X}^{\prime} \mid\{S=K\}$ has an $s$-concave density if $\boldsymbol{X}$ has. As shown in Dharmadhikari and Joag-Dev (1988) and Saumard and Wellner (2014), $s$-concavity is preserved under marginalization, convolution and weak-limit for certain ranges of $s \in \mathbb{R}$. Therefore, convex unimodality can also be preserved under these operations if $\boldsymbol{X}$ has the $s$-concave the density $f_{\boldsymbol{X}}$.

\section{Dependence of $\boldsymbol{X} \mid\{S=K\}$ and stochastic orders}

In this appendix, we investigate the dependence, especially the total positivity and its related orders of $\boldsymbol{X}^{\prime} \mid\{S=K\}$ implied by those of $\boldsymbol{X}$. To this end, we define the following concepts.

Definition 7 (Multivariate total positivity of order 2). Suppose random vectors $\boldsymbol{X}$ and $\boldsymbol{Y}$ have densities $f_{\boldsymbol{X}}$ and $f_{\boldsymbol{Y}}$, respectively.

1. $\boldsymbol{X}$ is said to be multivariate totally positively ordered of order 2 (MTP2) if

$$
f_{\boldsymbol{X}}(\boldsymbol{x}) f_{\boldsymbol{X}}(\boldsymbol{y}) \leq f_{\boldsymbol{X}}(\boldsymbol{x} \wedge \boldsymbol{y}) f_{\boldsymbol{X}}(\boldsymbol{x} \vee \boldsymbol{y}), \quad \text { for all } \boldsymbol{x}, \boldsymbol{y} \in \mathbb{R}^{d} .
$$

2. $\boldsymbol{X}$ is said to be multivariate reverse rule of order 2 (MRR2) if

$$
f_{\boldsymbol{X}}(\boldsymbol{x}) f_{\boldsymbol{X}}(\boldsymbol{y}) \geq f_{\boldsymbol{X}}(\boldsymbol{x} \wedge \boldsymbol{y}) f_{\boldsymbol{X}}(\boldsymbol{x} \vee \boldsymbol{y}), \quad \text { for all } \boldsymbol{x}, \boldsymbol{y} \in \mathbb{R}^{d} .
$$


3. $\boldsymbol{Y}$ is said to be larger than $\boldsymbol{X}$ in $T P 2$-order, denoted as $\boldsymbol{X} \leq_{t p} \boldsymbol{Y}$ if

$$
f_{\boldsymbol{X}}(\boldsymbol{x}) f_{\boldsymbol{Y}}(\boldsymbol{y}) \leq f_{\boldsymbol{X}}(\boldsymbol{x} \wedge \boldsymbol{y}) f_{\boldsymbol{Y}}(\boldsymbol{x} \vee \boldsymbol{y}), \quad \text { for all } \boldsymbol{x}, \boldsymbol{y} \in \mathbb{R}^{d}
$$

For examples and implied dependence properties of MTP2, MRR2 and TP2 ordered distributions, see Karlin and Rinott (1980a) and Karlin and Rinott (1980b). The following proposition states that the MTP2, MRR2 and TP2 order of $\boldsymbol{X}^{\prime} \mid\left\{\mathbf{1}_{d}^{\top} \boldsymbol{X}=K\right\}$ and $\boldsymbol{Y}^{\prime} \mid\left\{\mathbf{1}_{d}^{\top} \boldsymbol{Y}=K\right\}$ are inherited from those of $\left(\boldsymbol{X}^{\prime}, \mathbf{1}_{d}^{\top} \boldsymbol{X}\right)$ and $\left(\boldsymbol{Y}^{\prime}, \mathbf{1}_{d}^{\top} \boldsymbol{Y}\right)$.

Proposition 6 (MTP2, MRR2 and TP2 order of $\left.\boldsymbol{X}^{\prime} \mid\{S=K\}\right)$. Suppose $\left(\boldsymbol{X}^{\prime}, S\right)$ and $\left(\boldsymbol{Y}^{\prime}, T\right)$ with $S=\mathbf{1}_{d}^{\top} \boldsymbol{X}$ and $T=\mathbf{1}_{d}^{\top} \boldsymbol{Y}$ have densities $f_{\left(\boldsymbol{X}^{\prime}, S\right)}$ and $f_{\left(\boldsymbol{Y}^{\prime}, T\right)}$, respectively.

1. If $\left(\boldsymbol{X}^{\prime}, S\right)$ is MTP2 (MRR2) then $\boldsymbol{X}^{\prime} \mid\{S=K\}$ is MTP2 (MRR2).

2. If $\left(\boldsymbol{X}^{\prime}, S\right) \leq_{t p}\left(\boldsymbol{Y}^{\prime}, T\right)$ then $\boldsymbol{X}^{\prime}\left|\{S=K\} \leq_{t p} \boldsymbol{Y}^{\prime}\right|\{T=K\}$.

Proof. By (5) we have, for $\boldsymbol{x}^{\prime}, \boldsymbol{y}^{\prime} \in \mathbb{R}^{d^{\prime}}$, that

$$
\begin{aligned}
f_{\boldsymbol{X}^{\prime} \mid\{S=K\}}\left(\boldsymbol{x}^{\prime}\right) f_{\boldsymbol{X}^{\prime} \mid\{S=K\}}\left(\boldsymbol{y}^{\prime}\right) & =\frac{f_{\left(\boldsymbol{X}^{\prime}, S\right)}\left(\boldsymbol{x}^{\prime}, K\right) f_{\left(\boldsymbol{X}^{\prime}, S\right)}\left(\boldsymbol{y}^{\prime}, K\right)}{f_{S}^{2}(K)} \\
& \leq \frac{f_{\left(\boldsymbol{X}^{\prime}, S\right)}\left(\boldsymbol{x}^{\prime} \wedge \boldsymbol{y}^{\prime}, K \wedge K\right) f_{\left(\boldsymbol{X}^{\prime}, S\right)}\left(\boldsymbol{x}^{\prime} \vee \boldsymbol{y}^{\prime}, K \vee K\right)}{f_{S}^{2}(K)} \\
& =f_{\boldsymbol{X}^{\prime} \mid\{S=K\}}\left(\boldsymbol{x}^{\prime} \wedge \boldsymbol{y}^{\prime}\right) f_{\boldsymbol{X}^{\prime} \mid\{S=K\}}\left(\boldsymbol{x}^{\prime} \vee \boldsymbol{y}^{\prime}\right),
\end{aligned}
$$

which proves the first part on MTP2. The MRR2 and TP2 parts are shown in similar manners.

The properties of MTP2 (MRR2) and TP2 order have various implications. For example, when $\boldsymbol{X}^{\prime} \mid\{S=$ $K\}$ is MTP2, then $\boldsymbol{X}^{\prime} \mid\{S=K\}$ is positively associated in the sense that $\operatorname{Cov}\left[g\left(X_{i}\right), h\left(X_{j}\right) \mid\{S=K\}\right] \geq 0$ for all increasing functions $g: \mathbb{R} \rightarrow \mathbb{R}$ and $h: \mathbb{R} \rightarrow \mathbb{R}$. If $\boldsymbol{X}^{\prime}\left|\{S=K\} \leq_{t p} \boldsymbol{Y}^{\prime}\right|\{T=K\}$, then $\boldsymbol{X}^{\prime}\left|\{S=K\} \leq_{s t} \boldsymbol{Y}\right|\{T=K\}$, that is, $\mathbb{E}\left[h\left(\boldsymbol{X}^{\prime}\right) \mid\{S=K\}\right] \leq \mathbb{E}\left[h\left(\boldsymbol{Y}^{\prime}\right) \mid\{T=K\}\right]$ for all bounded and increasing functions $h: \mathbb{R}^{d^{\prime}} \rightarrow \mathbb{R}$. See Müller and Stoyan (2002) for more implications of the MTP2, MRR2 and TP2 order.

\section{Fallacies in risk allocations}

In this appendix we introduce two properties which intuitively hold but in general do not for the Euler and maximum likelihood allocations. For a $d$-dimensional random vector $\boldsymbol{X}$ and a real number $K \in \mathbb{R}$, an allocation principle $\boldsymbol{K}$ maps $(\boldsymbol{X}, K)$ to $\boldsymbol{K}(\boldsymbol{X} ; K) \in \mathbb{R}^{d}$ such that $\mathbf{1}_{d}^{\top} \boldsymbol{K}(\boldsymbol{X} ; K)=K$.

1. Invariance under independence:

For two integers $d, \tilde{d} \geq 2$, consider a $d$-dimensional random vector $\boldsymbol{X}$ with $S=\mathbf{1}_{d}^{\top} \boldsymbol{X}$ and a $\tilde{d}$ dimensional random vector $\tilde{\boldsymbol{X}}$ with $\tilde{S}=\mathbf{1}_{\tilde{d}}^{\top} \tilde{\boldsymbol{X}}$. For $K, \tilde{K}>0$, we call a risk allocation $\boldsymbol{K}$ invariant under independence if

$$
\boldsymbol{K}((\boldsymbol{X}, \tilde{\boldsymbol{X}}) ; K+\tilde{K})=(\boldsymbol{K}(\boldsymbol{X} ; K), \boldsymbol{K}(\tilde{\boldsymbol{X}} ; \tilde{K}))
$$


provided that $\boldsymbol{X}$ and $\tilde{\boldsymbol{X}}$ are independent of each other. This property means that risk allocation problems of multiple portfolios independent of each other can be considered separately. This property does not hold for MLA since the maximizers of the two functions

$$
\begin{aligned}
f_{(\boldsymbol{X}, \tilde{\boldsymbol{X}}) \mid\{S+\tilde{S}=K+\tilde{K}\}}((\boldsymbol{x}, \tilde{\boldsymbol{x}})) & =\frac{f_{(\boldsymbol{X}, \tilde{\boldsymbol{X}})}((\boldsymbol{x}, \tilde{\boldsymbol{x}})) \mathbf{1}_{\left\{\mathbf{1}_{d}^{\top} \boldsymbol{x}+\mathbf{1}_{\tilde{d}}^{\top} \tilde{\boldsymbol{x}}=K+\tilde{K}\right\}}}{f_{S+\tilde{S}}(K+\tilde{K})}=\frac{f_{\boldsymbol{X}}(\boldsymbol{x}) f_{\tilde{\boldsymbol{X}}}(\tilde{\boldsymbol{x}}) \mathbf{1}_{\left\{\mathbf{1}_{d}^{\top} \boldsymbol{x}+\mathbf{1}_{\tilde{d}}^{\top} \tilde{\boldsymbol{x}}=K+\tilde{K}\right\}}}{f_{S+\tilde{S}}(K+\tilde{K})} \\
& \propto f_{\boldsymbol{X}}(\boldsymbol{x}) f_{\tilde{\boldsymbol{X}}}(\tilde{\boldsymbol{x}}) \mathbf{1}_{\left\{\bigcup_{\left.\{k, \tilde{k}) \in \mathbb{R}^{2}, k+\tilde{k}=K+\tilde{K}\right\}}\left\{\mathbf{1}_{d}^{\top} \boldsymbol{x}=k\right\} \cap\left\{\mathbf{1}_{\tilde{d}} \tilde{\boldsymbol{x}}=\tilde{k}\right\}\right\}}
\end{aligned}
$$

and

$$
f_{\boldsymbol{X} \mid\{S=K\}}(\boldsymbol{x}) f_{\tilde{\boldsymbol{X}} \mid\{\tilde{S}=\tilde{K}\}}(\tilde{\boldsymbol{x}}) \propto f_{\boldsymbol{X}}(\boldsymbol{x}) \mathbf{1}_{\left\{\mathbf{1}_{d}^{\top} \boldsymbol{x}=K\right\}} f_{\tilde{\boldsymbol{X}}}(\tilde{\boldsymbol{x}}) \mathbf{1}_{\left\{\mathbf{1}_{\tilde{d}} \tilde{\boldsymbol{x}}=\tilde{K}\right\}}
$$

are in general different. For example, let $d=d^{\prime}$ and $\boldsymbol{X}$ and $\tilde{\boldsymbol{X}}$ be two independent and identically distributed standard normal distributions. Then the maximum of $(20)$ is attained at $(K+\tilde{K}) \mathbf{1}_{2 d} / 2 d$ whereas that of $(21)$ is attained at $\left(K \mathbf{1}_{d} / d, \tilde{K} \mathbf{1}_{d} / d\right)$. The two vectors are not equal unless $K=\tilde{K}$. In this example, the Euler allocation provides the same allocated capitals as MLA. Therefore, neither Euler allocation nor MLA satisfies invariance under independence.

\section{Additivity under convolution:}

Consider two independent $d$-dimensional random vectors $\boldsymbol{X}$ and $\tilde{\boldsymbol{X}}$ with $S=\mathbf{1}_{d}^{\top} \boldsymbol{X}$ and $\tilde{S}=\mathbf{1}_{d}^{\top} \tilde{\boldsymbol{X}}$. For $K, \tilde{K}>0$, we call an allocation $\boldsymbol{K}$ additive under convolution if

$$
\boldsymbol{K}(\boldsymbol{X}+\tilde{\boldsymbol{X}} ; K+\tilde{K})=\boldsymbol{K}(\boldsymbol{X} ; K)+\boldsymbol{K}(\tilde{\boldsymbol{X}} ; \tilde{K}) .
$$

Neither Euler allocation nor MLA satisfies this property. For example, let $\boldsymbol{X} \sim \mathrm{N}_{d}(\boldsymbol{\mu}, \Sigma)$ and $\tilde{\boldsymbol{X}} \sim \mathrm{N}_{d}(\tilde{\boldsymbol{\mu}}, \tilde{\Sigma})$ be two independent normal random vectors for $\boldsymbol{\mu}, \tilde{\boldsymbol{\mu}} \in \mathbb{R}^{d}$ and $\Sigma, \tilde{\Sigma} \in \mathcal{M}_{+}^{d \times d}$. By Proposition 1, Equation (7) and Proposition 2 Part 1, we have that

$$
\boldsymbol{K}_{\mathrm{M}}\left(\boldsymbol{X} ; \mathcal{K}_{d}(K)\right)=\boldsymbol{\mu}^{\prime}+\frac{K-\mu_{S}}{\sigma_{S}^{2}}\left(\Sigma \mathbf{1}_{d}\right)^{\prime} \quad \text { and } \quad \boldsymbol{K}_{\mathrm{M}}\left(\tilde{\boldsymbol{X}} ; \mathcal{K}_{d}(\tilde{K})\right)=\tilde{\boldsymbol{\mu}}^{\prime}+\frac{\tilde{K}-\mu_{\tilde{S}}}{\sigma_{\tilde{S}}^{2}}\left(\tilde{\Sigma} \mathbf{1}_{d}\right)^{\prime}
$$

Similarly, since $\boldsymbol{X}+\tilde{\boldsymbol{X}} \sim \mathrm{N}_{d}(\boldsymbol{\mu}+\tilde{\boldsymbol{\mu}}, \Sigma+\tilde{\Sigma})$, we have that $\sigma_{S+\tilde{S}}^{2}=\sigma_{S}^{2}+\sigma_{\tilde{S}}^{2}$ and that

$$
\begin{aligned}
\boldsymbol{K}_{\mathrm{M}}\left(\boldsymbol{X}+\tilde{\boldsymbol{X}} ; \mathcal{K}_{d}(K+\tilde{K})\right) & =\boldsymbol{\mu}^{\prime}+\tilde{\boldsymbol{\mu}}^{\prime}+\frac{K+\tilde{K}-\left(\mu_{S}+\mu_{\tilde{S}}\right)}{\sigma_{S}^{2}+\sigma_{\tilde{S}}^{2}}\left((\Sigma+\tilde{\Sigma}) \mathbf{1}_{d}\right)^{\prime} \\
& =\boldsymbol{\mu}^{\prime}+\tilde{\boldsymbol{\mu}}^{\prime}+\left(\frac{\sigma_{S}^{2}}{\sigma_{S}^{2}+\sigma_{\tilde{S}}^{2}} \frac{K-\mu_{S}}{\sigma_{S}^{2}}+\frac{\sigma_{\tilde{S}}^{2}}{\sigma_{S}^{2}+\sigma_{\tilde{S}}^{2}} \frac{\tilde{K}-\mu_{\tilde{S}}}{\sigma_{\tilde{S}}^{2}}\right)\left((\Sigma+\tilde{\Sigma}) \mathbf{1}_{d}\right)^{\prime}
\end{aligned}
$$

which is not equal to $\boldsymbol{K}_{\mathrm{M}}\left(\boldsymbol{X} ; \mathcal{K}_{d}(K)\right)+\boldsymbol{K}_{\mathrm{M}}\left(\tilde{\boldsymbol{X}} ; \mathcal{K}_{d}(\tilde{K})\right)$ unless, for instance, $\Sigma=\tilde{\Sigma}$. Since Euler and maximum likelihood allocations coincide under ellipticality, the same statement holds for Euler allocations.

\section{E Further properties of the multimodality adjustment}

In this section we study further properties of the multimodality adjustment $\boldsymbol{K}_{\boldsymbol{w}, \mathcal{X}, \Lambda}$ introduced in Section 4.2. To clarify the relationship between $\boldsymbol{K}_{\boldsymbol{w}, \mathcal{X}, \Lambda}$, the total capital $K$ and the loss distribution of $\boldsymbol{X}$, 
define $\boldsymbol{K}_{\boldsymbol{w}, \mathcal{X}, \Lambda}\left[\boldsymbol{X} ; \mathcal{K}_{d}(K)\right]$ and $\overline{\boldsymbol{K}}_{\boldsymbol{w}, \mathcal{X}}\left[\boldsymbol{X} ; \mathcal{K}_{d}(K)\right]$ to be the multimodality-adjusted allocated capitals (14) and their first term $\sum_{m=1}^{M} w_{m} \boldsymbol{K}_{m}$, respectively, with $\mathcal{X}$ being the set of local modes $\boldsymbol{K}_{1}, \ldots, \boldsymbol{K}_{M}$ of $\boldsymbol{x} \mapsto$ $f_{\boldsymbol{X}}(\boldsymbol{x}) \mathbf{1}_{\left\{\boldsymbol{x} \in \mathcal{K}_{d}(K)\right\}}$ (assumed to be a finite set) and with $w_{m} \propto f_{\boldsymbol{X}}\left(\boldsymbol{K}_{m}\right)$. To this end, we adopt the following definition of local modes.

Definition 8 (Local modes). For an $\mathbb{R}_{+}$-valued function $f$ on $\mathbb{R}^{d}, \boldsymbol{x} \in \mathbb{R}^{d}$ is called a local mode of $f$ if there exists $\epsilon>0$ such that

$$
f(\boldsymbol{x}) \geq f(\boldsymbol{y}) \text { for all } \boldsymbol{y} \in \mathcal{N}_{\epsilon}(\boldsymbol{x})
$$

where $\mathcal{N}_{\epsilon}(\boldsymbol{x})=\left\{\boldsymbol{z} \in \mathbb{R}^{d}:\|\boldsymbol{z}-\boldsymbol{x}\|<\epsilon\right\}$. If (22) holds for any $\epsilon>0$, then $\boldsymbol{x}$ is called a global mode of $f$.

Properties of $\boldsymbol{K}_{\boldsymbol{w}, \mathcal{X}, \Lambda}\left[\boldsymbol{X} ; \mathcal{K}_{d}(K)\right]$ are then summarized as follows.

1. Translation invariance: We show that $\boldsymbol{K}_{\boldsymbol{w}, \mathcal{X}, \Lambda}$ is translation invariant in the sense that

$$
\boldsymbol{K}_{\boldsymbol{w}, \mathcal{X}, \Lambda}\left[\boldsymbol{X}+\boldsymbol{c} ; \mathcal{K}_{d}\left(K+\mathbf{1}_{d}^{\top} \boldsymbol{c}\right)\right]=\boldsymbol{K}_{\boldsymbol{w}, \mathcal{X}, \Lambda}\left[\boldsymbol{X} ; \mathcal{K}_{d}(K)\right]+\boldsymbol{c} \quad \text { for } \quad \boldsymbol{c} \in \mathbb{R}^{d}
$$

To show this, notice that local modes of $\boldsymbol{x} \mapsto f_{\boldsymbol{X}+\boldsymbol{c}}(\boldsymbol{x}) \mathbf{1}_{\left\{\boldsymbol{x} \in \mathcal{K}_{d}\left(K+\mathbf{1}_{d}^{\top} \boldsymbol{c}\right)\right\}}$ are given by $\boldsymbol{K}_{m}+\boldsymbol{c}, m=$ $1, \ldots, M$, if $\boldsymbol{K}_{m}, m=1, \ldots, M$, are the local modes of $\boldsymbol{x} \mapsto f_{\boldsymbol{X}}(\boldsymbol{x}) \mathbf{1}_{\left\{\boldsymbol{x} \in \mathcal{K}_{d}(K)\right\}}$. Since $w_{m}=$ $f_{\boldsymbol{X}}\left(\boldsymbol{K}_{m}\right)=f_{\boldsymbol{X}+\boldsymbol{c}}\left(\boldsymbol{K}_{m}+\boldsymbol{c}\right)$, the probability weight assigned to the $m$ th scenario does not change from $\left(\boldsymbol{X}, \mathcal{K}_{d}(K)\right)$ to $\left(\boldsymbol{X}+\boldsymbol{c}, \mathcal{K}_{d}\left(K+\mathbf{1}_{d}^{\top} \boldsymbol{c}\right)\right)$ for all $m=1, \ldots, M$. Therefore, $\overline{\boldsymbol{K}}_{\boldsymbol{w}, \mathcal{X}}\left[\boldsymbol{X}+\boldsymbol{c} ; \mathcal{K}_{d}\left(K+\mathbf{1}_{d}^{\top} \boldsymbol{c}\right)\right]=$ $\overline{\boldsymbol{K}}_{\boldsymbol{w}, \mathcal{X}}\left[\boldsymbol{X} ; \mathcal{K}_{d}(K)\right]+\boldsymbol{c}$ and thus

$$
\begin{aligned}
\boldsymbol{K}_{\boldsymbol{w}, \mathcal{X}, \Lambda}\left[\boldsymbol{X}+\boldsymbol{c} ; \mathcal{K}_{d}\left(K+\mathbf{1}_{d}^{\top} \boldsymbol{c}\right)\right]= & \overline{\boldsymbol{K}}_{\boldsymbol{w}, \mathcal{X}}\left[\boldsymbol{X}+\boldsymbol{c} ; \mathcal{K}_{d}\left(K+\mathbf{1}_{d}^{\top} \boldsymbol{c}\right)\right] \\
& +\sum_{m=1}^{M} w_{m} \boldsymbol{\lambda}_{m} \circ\left(\boldsymbol{K}_{m}+\boldsymbol{c}-\overline{\boldsymbol{K}}_{\boldsymbol{w}, \mathcal{X}}\left[\boldsymbol{X}+\boldsymbol{c} ; \mathcal{K}_{d}\left(K+\mathbf{1}_{d}^{\top} \boldsymbol{c}\right)\right]\right)^{+} \\
= & \overline{\boldsymbol{K}}_{\boldsymbol{w}, \mathcal{X}}\left[\boldsymbol{X} ; \mathcal{K}_{d}(K)\right]+\boldsymbol{c}+\sum_{m=1}^{M} w_{m} \boldsymbol{\lambda}_{m} \circ\left(\boldsymbol{K}_{m}-\overline{\boldsymbol{K}}_{\boldsymbol{w}, \mathcal{X}}\left[\boldsymbol{X} ; \mathcal{K}_{d}(K)\right]\right)^{+} \\
= & \boldsymbol{K}_{\boldsymbol{w}, \mathcal{X}, \Lambda}\left[\boldsymbol{X} ; \mathcal{K}_{d}(K)\right]+\boldsymbol{c},
\end{aligned}
$$

which shows translation invariance.

2. Positive homogeneity: Multimodality-adjusted allocated capitals are positive homogeneous in the sense that

$$
\boldsymbol{K}_{\boldsymbol{w}, \mathcal{X}, \Lambda}\left[c \boldsymbol{X} ; \mathcal{K}_{d}(c K)\right]=c \boldsymbol{K}_{\boldsymbol{w}, \mathcal{X}, \Lambda}\left[\boldsymbol{X} ; \mathcal{K}_{d}(K)\right] \quad \text { for } \quad c>0
$$

This can be checked similarly as translation invariance. The local modes of $\boldsymbol{x} \mapsto f_{c \boldsymbol{X}}(\boldsymbol{x}) \mathbf{1}_{\left\{\boldsymbol{x} \in \mathcal{K}_{d}(c K)\right\}}$ are given by $c \boldsymbol{K}_{m}, m=1, \ldots, M$, if $\boldsymbol{K}_{m}, m=1, \ldots, M$, are the local modes of $\boldsymbol{x} \mapsto f_{\boldsymbol{X}}(\boldsymbol{x}) \mathbf{1}_{\left\{\boldsymbol{x} \in \mathcal{K}_{d}(K)\right\}}$. Moreover, the probability weight assigned to the $m$ th scenario does not change from $\left(\boldsymbol{X}, \mathcal{K}_{d}(K)\right)$ to $\left(c \boldsymbol{X}, \mathcal{K}_{d}(c K)\right)$ since $w_{m}=f_{\boldsymbol{X}}\left(\boldsymbol{K}_{m}\right)=f_{c \boldsymbol{X}}\left(c \boldsymbol{K}_{m}\right)$ for all $m=1, \ldots, M$. Therefore, $\overline{\boldsymbol{K}}_{\boldsymbol{w}, \mathcal{X}}\left[c \boldsymbol{X} ; \mathcal{K}_{d}(c K)\right]=$ 
$c \overline{\boldsymbol{K}}_{\boldsymbol{w}, \mathcal{X}}\left[\boldsymbol{X} ; \mathcal{K}_{d}(K)\right]$ and thus

$$
\begin{aligned}
\boldsymbol{K}_{\boldsymbol{w}, \mathcal{X}, \Lambda}\left[c \boldsymbol{X} ; \mathcal{K}_{d}(c K)\right]= & \overline{\boldsymbol{K}}_{\boldsymbol{w}, \mathcal{X}}\left[c \boldsymbol{X} ; \mathcal{K}_{d}(c K)\right] \\
& +\sum_{m=1}^{M} w_{m} \boldsymbol{\lambda}_{m} \circ\left(c \boldsymbol{K}_{m}-\overline{\boldsymbol{K}}_{\boldsymbol{w}, \mathcal{X}}\left[c \boldsymbol{X} ; \mathcal{K}_{d}(c K)\right]\right)^{+} \\
= & c \overline{\boldsymbol{K}}_{\boldsymbol{w}, \mathcal{X}}\left[\boldsymbol{X} ; \mathcal{K}_{d}(K)\right]+c \sum_{m=1}^{M} w_{m} \boldsymbol{\lambda}_{m} \circ\left(\boldsymbol{K}_{m}-\overline{\boldsymbol{K}}_{\boldsymbol{w}, \mathcal{X}}\left[\boldsymbol{X} ; \mathcal{K}_{d}(K)\right]\right)^{+} \\
= & c \boldsymbol{K}_{\boldsymbol{w}, \mathcal{X}, \Lambda}\left[\boldsymbol{X} ; \mathcal{K}_{d}(K)\right],
\end{aligned}
$$

which shows positive homogeneity.

3. Riskless asset: Multimodality-adjusted allocated capitals satisfy the riskless asset property in the following sense. Suppose that $X_{j}=c_{j} \in \mathbb{R}$ a.s. for $j \in I \subseteq\{1, \ldots, d\}$ and that $\boldsymbol{X}_{-I}=\left(X_{j}, j \in\right.$ $\{1, \ldots, d\} \backslash I)$ admits a density $f_{\boldsymbol{X}_{-I}}$. As we discussed in Section 4.1.2, any realization $\boldsymbol{x}$ of $\boldsymbol{X} \mid\{S=$ $K\}$ satisfies $\boldsymbol{x}_{I}=\boldsymbol{c}$ where $\boldsymbol{c}=\left(c_{j} ; j \in I\right)$, and the likelihood of $\boldsymbol{x}$ is quantified through the density $f_{\boldsymbol{X}_{-I} \mid\left\{\mathbf{1}_{|-I|}^{\top} \boldsymbol{X}_{-I}=K-\mathbf{1}_{|I|}^{\top} \boldsymbol{c}\right\}}\left(\boldsymbol{x}_{-I}\right)$. Therefore, reasonable choices of the scenarios $\boldsymbol{K}_{1}, \ldots, \boldsymbol{K}_{M} \in \mathcal{K}_{d}(K)$ are such that $\left(\boldsymbol{K}_{m}\right)_{I}=\boldsymbol{c}$ and $\left(\boldsymbol{K}_{m}\right)_{-I}$ are local modes of $\boldsymbol{X}_{-I} \mid\left\{\mathbf{1}_{|-I|}^{\top} \boldsymbol{X}_{-I}=K-\mathbf{1}_{|I|}^{\top} \boldsymbol{c}\right\}$. In this case, we have that $\left(\overline{\boldsymbol{K}}_{\boldsymbol{w}, \mathcal{X}}\right)_{I}=\boldsymbol{c}$ and the multimodality adjustment yields

$$
\left(\sum_{m=1}^{M} w_{m} \boldsymbol{\lambda}_{m} \circ\left(\boldsymbol{K}_{m}-\bar{K}_{\boldsymbol{w}, \mathcal{X}}\right)^{+}\right)_{I}=\sum_{m=1}^{M} w_{m}\left(\boldsymbol{\lambda}_{m}\right)_{I} \circ(\boldsymbol{c}-\boldsymbol{c})^{+}=\mathbf{0}_{|I|} .
$$

Therefore, it holds that $\boldsymbol{K}_{\boldsymbol{w}, \mathcal{X}, \Lambda}\left[\boldsymbol{X} ; \mathcal{K}_{d}(K)\right]_{I}=\boldsymbol{c}$ if $\boldsymbol{X}_{I}=\boldsymbol{c}$ a.s.

4. Symmetry: For a reasonable choice of $\Lambda$, the multimodality-adjusted allocated capitals satisfy the symmetry property, that is, $\boldsymbol{K}_{\boldsymbol{w}, \mathcal{X}, \Lambda}\left[\boldsymbol{X} ; \mathcal{K}_{d}(K)\right]_{i}=\boldsymbol{K}_{\boldsymbol{w}, \mathcal{X}, \Lambda}\left[\boldsymbol{X} ; \mathcal{K}_{d}(K)\right]_{j}$ for $i, j \in\{1, \ldots, d\}, i \neq j$, if $\boldsymbol{X} \stackrel{\text { d }}{=} \tilde{\boldsymbol{X}}$ where $\tilde{\boldsymbol{X}}$ is a $d$-dimensional random vector satisfying $\tilde{X}_{j}=X_{i}, \tilde{X}_{i}=X_{j}$ and $\tilde{X}_{k}=X_{k}$ for all $k \in\{1, \ldots, d\} \backslash\{i, j\}$. For any $\boldsymbol{x} \in \mathbb{R}^{d}$, denote by $\tilde{\boldsymbol{x}}$ a $d$-dimensional vector such that $\tilde{x}_{j}=x_{i}$, $\tilde{x}_{i}=x_{j}$ and $\tilde{x}_{k}=x_{k}$ for all $k \in\{1, \ldots, d\} \backslash\{i, j\}$. To show the symmetry, suppose that $\boldsymbol{K} \in \mathcal{K}_{d}(K)$ is a local mode of $\boldsymbol{x} \mapsto f_{\boldsymbol{X}}(\boldsymbol{x}) \mathbf{1}_{\left\{\boldsymbol{x} \in \mathcal{K}_{d}(K)\right\}}$. Then $\tilde{\boldsymbol{K}}$ is also a local mode of $\boldsymbol{x} \mapsto f_{\boldsymbol{X}}(\boldsymbol{x}) \mathbf{1}_{\left\{\boldsymbol{x} \in \mathcal{K}_{d}(K)\right\}}$ since $f_{\boldsymbol{X}}(\boldsymbol{y})=f_{\tilde{\boldsymbol{X}}}(\tilde{\boldsymbol{y}})=f_{\boldsymbol{X}}(\tilde{\boldsymbol{y}})$ for any $\boldsymbol{y} \in \mathbb{R}^{d}$ by $\boldsymbol{X} \stackrel{\mathrm{d}}{=} \tilde{\boldsymbol{X}}$, and thus $\tilde{\boldsymbol{K}}$ satisfies (22) for some $\epsilon>0$. Therefore, any element $\boldsymbol{K}_{m}=\left(K_{m, 1}, \ldots, K_{m, d}\right)$ in $\mathcal{X}$ satisfies either (1) $K_{m, i}=K_{m, j}$ or (2) there exists a unique element $\boldsymbol{K}_{m^{\prime}} \in \mathcal{X}$ such that $K_{m, i} \neq K_{m, j}, K_{m^{\prime}, i} \neq K_{m^{\prime}, j}, K_{m^{\prime}, i}=K_{m, j}$, $K_{m, i}=K_{m^{\prime}, j}$ and $K_{m, k}=K_{m^{\prime}, k}$ for all $k \in\{1, \ldots, d\} \backslash\{i, j\}$. For such a pair of indices $\left(m, m^{\prime}\right)$, it holds that $w_{m}=w_{m^{\prime}}$ since $w_{m} \propto f_{\boldsymbol{X}}\left(\boldsymbol{K}_{m}\right)$ and $w_{m^{\prime}} \propto f_{\boldsymbol{X}}\left(\boldsymbol{K}_{m^{\prime}}\right)=f_{\tilde{\boldsymbol{X}}}\left(\boldsymbol{K}_{m}\right)=f_{\boldsymbol{X}}\left(\boldsymbol{K}_{m}\right)$ by $\boldsymbol{X} \stackrel{\mathrm{d}}{=} \tilde{\boldsymbol{X}}$. Therefore, if $\boldsymbol{\lambda}_{m}=\boldsymbol{\lambda}_{m^{\prime}}$ holds for all pairs of $\left(m, m^{\prime}\right)$, then the symmetry $\boldsymbol{K}_{\boldsymbol{w}, \mathcal{X}, \Lambda}\left[\boldsymbol{X} ; \mathcal{K}_{d}(K)\right]_{i}=$ $\boldsymbol{K}_{\boldsymbol{w}, \mathcal{X}, \Lambda}\left[\boldsymbol{X} ; \mathcal{K}_{d}(K)\right]_{j}$ holds.

The continuity property $\lim _{n \rightarrow \infty} \boldsymbol{K}_{\boldsymbol{w}, \mathcal{X}, \Lambda}\left[\boldsymbol{X}_{n} ; \mathcal{K}_{d}(K)\right]=\boldsymbol{K}_{\boldsymbol{w}, \mathcal{X}, \Lambda}\left[\boldsymbol{X} ; \mathcal{K}_{d}(K)\right]$ for a given $\boldsymbol{X}_{n}$ and $\boldsymbol{X}$ such that $\boldsymbol{X}_{n}$ converges to $\boldsymbol{X}$ weakly may not be straightforward to verify since a limit of multimodal distributions can be unimodal, and more generally, the number of scenarios may change in $n$. 


\section{F Simulation of $\boldsymbol{X} \mid\{S=K\}$ with MCMC methods}

Efficient simulation of the conditional distribution of $\boldsymbol{X}$ given a constant sum $\{S=K\}$ for $K \in \mathbb{R}$ is a challenging task in general. In Section 2.2 and Section 5 , the constraint $\{S=K\}$ was replaced by $\{K-\delta<S<K+\delta\}$ for a small $\delta>0$ so that $\mathbb{P}(K-\delta<S<K+\delta)>0$. However, this modification distorts the conditional distribution $\boldsymbol{X} \mid\{S=K\}$ and the resulting estimates of risk allocations suffer from inevitable biases. To overcome this issue, we briefly review MCMC methods, specifically the MetropolisHastings (MH) algorithm, and then demonstrate their efficiency for simulating $\boldsymbol{X} \mid\{S=K\}$.

\section{F.1 MCMC methods}

As mentioned in Section 2.1, it suffices to simulate $\boldsymbol{X}^{\prime}\left|\{S=K\}=\left(X_{1}, \ldots, X_{d^{\prime}}\right)\right|\{S=K\}$ for $d^{\prime}=d-1$. Assume that $\boldsymbol{X}^{\prime} \mid\{S=K\}$ admits a density (5). We call this density the target density and denote it as $\pi$. In the MCMC approach, a Markov chain is constructed such that its stationary distribution is $\pi$. Constructing such a Markov chain can be achieved by the MH algorithm as we now explain. From the current state $\boldsymbol{X}_{n}^{\prime}$, a candidate $\boldsymbol{Y}_{n}^{\prime}$ of the next state is simulated from $q\left(\boldsymbol{X}_{n}^{\prime}, \cdot\right)$ where $q\left(\boldsymbol{x}^{\prime}, \boldsymbol{y}^{\prime}\right), \boldsymbol{x}^{\prime}, \boldsymbol{y}^{\prime} \in \mathbb{R}^{d^{\prime}}$ is called the proposal density satisfying the two conditions that (i) $\boldsymbol{x}^{\prime} \mapsto q\left(\boldsymbol{x}^{\prime}, \boldsymbol{y}^{\prime}\right)$ is measurable for all $\boldsymbol{y}^{\prime} \in \mathbb{R}^{d^{\prime}}$, and (ii) $\boldsymbol{y}^{\prime} \mapsto q\left(\boldsymbol{x}^{\prime}, \boldsymbol{y}^{\prime}\right)$ is a density function for all $\boldsymbol{x}^{\prime} \in \mathbb{R}^{d^{\prime}}$. The candidate is accepted, that is, $\boldsymbol{X}_{n+1}^{\prime}=\boldsymbol{Y}_{n}^{\prime}$, with probability $\alpha\left(\boldsymbol{X}_{n}^{\prime}, \boldsymbol{Y}_{n}^{\prime}\right)$ where

$$
\alpha\left(\boldsymbol{x}^{\prime}, \boldsymbol{y}^{\prime}\right)=1 \wedge \frac{q\left(\boldsymbol{x}^{\prime}, \boldsymbol{y}^{\prime}\right) \pi\left(\boldsymbol{y}^{\prime}\right)}{q\left(\boldsymbol{y}^{\prime}, \boldsymbol{x}^{\prime}\right) \pi\left(\boldsymbol{x}^{\prime}\right)}=1 \wedge \frac{q\left(\boldsymbol{x}^{\prime}, \boldsymbol{y}^{\prime}\right) f_{\boldsymbol{X}}\left(\boldsymbol{y}^{\prime}, K-\mathbf{1}_{d^{\prime}}^{\top} \boldsymbol{y}^{\prime}\right)}{q\left(\boldsymbol{y}^{\prime}, \boldsymbol{x}^{\prime}\right) f_{\boldsymbol{X}}\left(\boldsymbol{x}^{\prime}, K-\mathbf{1}_{d^{\prime}}^{\top} \boldsymbol{x}^{\prime}\right)},
$$

and otherwise the chain stays at the current state $\boldsymbol{X}_{n+1}^{\prime}=\boldsymbol{X}_{n}^{\prime}$. Calculation of the acceptance probability (23) is often possible since it does not depend on $f_{S}(K)$. The resulting Markov chain is shown to have $\pi$ as a stationary distribution and thus $\boldsymbol{X}_{1}^{\prime}, \boldsymbol{X}_{2}^{\prime}, \ldots$ can be used as samples from $\pi$ in order to estimate Euler and maximum likelihood allocations.

An appropriate choice of $q$ is important since MCMC samples are typically positively correlated due to the acceptance-rejection procedure. To reduce positive correlation among MCMC samples, $q$ must reflect properties of $\pi$, such as the shape of its support, modality and tail behavior, for maintaining high acceptance probability $\alpha$. First, the support of $\pi$ must be taken into account since a candidate outside of the support of $\pi$ is immediately rejected. Second, heavy-tailed target distribution $\pi$ requires specific design of $q$ since most standard MCMC methods such as random walk MH, independent MH, Gibbs samplers and the Hamiltonian Monte Carlo methods cannot guarantee the theoretical convergence when $\pi$ is heavy-tailed. Finally, multimodality of $\pi$ also requires to be handled specifically since the chain needs to traverse from one mode to another to sample from the entire support of $\pi$.

\section{F.2 An application of MCMC to core allocation}

In this section we compute the Euler allocation and MLA on the restricted set of allocations called the (atomic) core defined by

$$
\mathcal{K}_{d}^{\mathrm{C}}(K ; r)=\left\{\boldsymbol{x} \in \mathbb{R}^{d}: \mathbf{1}_{d}^{\top} \boldsymbol{x}=K, \boldsymbol{\lambda}^{\top} \boldsymbol{x} \leq r(\boldsymbol{\lambda}), \boldsymbol{\lambda} \in\{0,1\}^{d}\right\} \subseteq \mathcal{K}_{d}(K),
$$

where $K$ is a given total capital and $r:\{0,1\}^{d} \rightarrow \mathbb{R}$ is called a participation profile function typically determined as $r(\boldsymbol{\lambda})=\varrho\left(\boldsymbol{\lambda}^{\top} \boldsymbol{X}\right)$ for a $d$-dimensional loss random vector $\boldsymbol{X}$ and a risk measure $\varrho$. We call an 
element of $\mathcal{K}_{d}^{\mathrm{C}}(K ; r)$ a core allocation. As explained in Denault $(2001)$, core allocations possess an important property as risk allocations, that is, any subportfolio of $\boldsymbol{X}=\left(X_{1}, \ldots, X_{d}\right)$ of the form $\left(\lambda_{1} X_{1}, \ldots, \lambda_{d} X_{d}\right)$ gains benefit of capital reduction by managing risk as a portfolio $\boldsymbol{X}$. In fact, for a participation profile $\boldsymbol{\lambda}=\left(\lambda_{1}, \ldots, \lambda_{d}\right)$ where $\lambda_{j} \in\{0,1\}$ represents the presence $\left(\lambda_{j}=1\right)$ or the absence $\left(\lambda_{j}=0\right)$ of the $j$ th entity, the total amount of capital required to cover the loss $\boldsymbol{\lambda}^{\top} \boldsymbol{X}$ is $\boldsymbol{\lambda}^{\top} \boldsymbol{x}$ for an allocation $\boldsymbol{x} \in \mathcal{K}_{d}(K)$. The value $r(\lambda)=\varrho\left(\boldsymbol{\lambda}^{\top} \boldsymbol{X}\right)$ is interpreted as a stand-alone capital that would have been required if the total loss $\boldsymbol{\lambda}^{\top} \boldsymbol{X}$ had been managed individually. Therefore, under the core allocation $\boldsymbol{x} \in \mathcal{K}_{d}^{\mathrm{C}}(K ; r)$, the subportfolio $\left(\lambda_{1} X_{1}, \ldots, \lambda_{d} X_{d}\right)$ gains benefit of capital reduction by $\boldsymbol{\lambda}^{\top} \boldsymbol{x}$ in comparison to $r(\boldsymbol{\lambda})$.

Given $K, r$ and the joint loss $\boldsymbol{X}$, we are interested in calculating the core-compatible versions of Euler allocation $\mathbb{E}\left[\boldsymbol{X} \mid\left\{\boldsymbol{X} \in \mathcal{K}_{d}^{\mathrm{C}}(K ; r)\right\}\right]$, MLA $\boldsymbol{K}_{\mathrm{M}}\left[\boldsymbol{X} ; \mathcal{K}_{d}^{\mathrm{C}}(K ; r)\right]$ and local modes of $f_{\boldsymbol{X} \mid\left\{\boldsymbol{X} \in \mathcal{K}_{d}^{\mathrm{C}}(K ; r)\right\}}$ if they exist. However, generating a large number of samples from $\boldsymbol{X}^{\prime} \mid\left\{\boldsymbol{X} \in \mathcal{K}_{d}^{\mathrm{C}}(K ; r)\right\}$ is computationally involved since an unconditional sample $\boldsymbol{X}$ is first filtered by the condition $\boldsymbol{X} \in\left\{\boldsymbol{x} \in \mathbb{R}^{d}: K-\delta<\mathbf{1}_{d}^{\top} \boldsymbol{x}<K+\delta\right\}=\mathcal{K}_{d}(K, \delta)$ for a small $\delta>0$, and then filtered again by the core condition $\boldsymbol{\lambda}^{\top} \boldsymbol{X} \leq r(\boldsymbol{\lambda})$ for all possible $\boldsymbol{\lambda} \in\{0,1\}^{d}$. To overcome this issue, we utilize the Hamiltonian Monte Carlo (HMC) method with reflection to directly simulate $f_{\boldsymbol{X}^{\prime} \mid\left\{\boldsymbol{X} \in \mathcal{K}_{d}^{\mathrm{C}}(K ; r)\right\}}$. Note that the support of $\boldsymbol{X}^{\prime} \mid\left\{\boldsymbol{X} \in \mathcal{K}_{d}^{\mathrm{C}}(K ; r)\right\}$ is a projection of $\mathcal{K}_{d}^{\mathrm{C}}(K ; r)$ onto $\mathbb{R}^{d^{\prime}}$, which is an intersection of finite number of hyperplanes $\left\{\boldsymbol{x}^{\prime} \in \mathbb{R}^{d^{\prime}}: \boldsymbol{\lambda}^{\top}\left(\boldsymbol{x}^{\prime}, K-\mathbf{1}_{d^{\prime}}^{\top} \boldsymbol{x}^{\prime}\right) \leq r(\boldsymbol{\lambda})\right\}$ for $\boldsymbol{\lambda} \in\{0,1\}^{d}$. In the HMC method, a candidate is proposed according to the so-called Hamiltonian dynamics, and the chain reflects at the boundaries $\left\{\boldsymbol{x}^{\prime} \in \mathbb{R}^{d^{\prime}}: \boldsymbol{\lambda}^{\top}\left(\boldsymbol{x}^{\prime}, K-\mathbf{1}_{d^{\prime}}^{\top} \boldsymbol{x}^{\prime}\right)=r(\boldsymbol{\lambda})\right\}, \boldsymbol{\lambda} \in\{0,1\}^{d}$, so that it does not violate the support constraint; see Koike and Hofert (2020) for details.

For a numerical experiment, let $\boldsymbol{X} \sim t_{\nu}\left(\mathbf{0}_{d}, P\right)$ with $d=3, \nu=5$ and $P=\left(\rho_{i j}\right)$ being a correlation matrix with $\rho_{12}=\rho_{23}=1 / 3$ and $\rho_{13}=2 / 3$. For $p=0.99$, we set $r(\boldsymbol{\lambda})=\operatorname{VaR}_{p}\left(\boldsymbol{\lambda}^{\top} \boldsymbol{X}\right)$ for $\boldsymbol{\lambda} \in\{0,1\}^{3}$ and $K=r\left(\mathbf{1}_{3}\right)$. For $\delta=0.001$, we first generate $N_{\mathrm{MC}}=10^{6}$ samples from $\boldsymbol{X}$ and estimate $K$ and $\left(r(\boldsymbol{\lambda}), \boldsymbol{\lambda} \in\{0,1\}^{3}\right)$ from these samples. Then we extract samples of $\boldsymbol{X}$ falling in the region

$$
\mathcal{K}_{d}^{\mathrm{C}}(K, \delta ; r)=\mathcal{K}_{d}(K, \delta) \cap\left\{\boldsymbol{x} \in \mathbb{R}^{d}: \boldsymbol{\lambda}^{\top} \boldsymbol{x} \leq r(\boldsymbol{\lambda}), \boldsymbol{\lambda} \in\{0,1\}^{3} \backslash\left\{\mathbf{1}_{3}\right\}\right\} .
$$

Figure 4 (a) shows the first two components of the MC samples from $\boldsymbol{X}$ and the conditional samples falling in $\mathcal{K}_{d}^{\mathrm{C}}(K, \delta ; r)$. Among the $N_{\mathrm{MC}}=10^{6}$ samples, 2000 samples were contained in $\mathcal{K}_{d}(K, \delta)$ and only 189 samples fell in $\mathcal{K}_{d}^{\mathrm{C}}(K, \delta ; r)$. Therefore, this crude simulation method is not efficient since $99.98 \%$ of the unconditional samples are discarded.

Instead, we conduct an MCMC simulation to generate $N_{\mathrm{MCMC}}=10^{4}$ samples directly from $\boldsymbol{X} \mid\{\boldsymbol{X} \in$ $\left.\mathcal{K}_{d}^{\mathrm{C}}(K ; r)\right\}$. Hyperparameters of the HMC method are estimated based on the $189 \mathrm{MC}$ samples; see Koike and Hofert (2020)for details. The resulting stepsize and integration time are $\varepsilon=0.105$ and $T=24$, respectively. It took 49.534 seconds to simulate a Markov chain with length $N_{\mathrm{MCMC}}=10^{4}$ on a MacBook Air with $1.4 \mathrm{GHz}$ Intel Core i5 processor and $4 \mathrm{~GB} 1600 \mathrm{MHz}$ of DDR3 RAM. The resulting acceptance rate was 0.866 and serial correlations were below 0.03 at lag 1 . Based on these inspections, we conclude that the MCMC method performed correctly. The first 3000 MCMC samples of $\boldsymbol{X}^{\prime} \mid\left\{\boldsymbol{X} \in \mathcal{K}_{d}^{\mathrm{C}}(K ; r)\right\}$ are plotted in Figure 4 (b).

By Proposition 1, $\boldsymbol{X}^{\prime} \mid\left\{\boldsymbol{X} \in \mathcal{K}_{d}(K)\right\}$ follows a multivariate Student $t$ distribution, and thus the mode of this conditional distribution is uniquely determined by $\boldsymbol{K}_{\mathrm{M}}\left[\boldsymbol{X} ; \mathcal{K}_{d}(K)\right]=\mathbb{E}\left[\boldsymbol{X} \mid\left\{\boldsymbol{X} \in \mathcal{K}_{d}(K)\right\}\right]$ by Proposition 2 Part 1 . Moreover, when this point is contained in the core $\boldsymbol{K}_{d}^{\mathrm{C}}(K ; r)$, we have that $\boldsymbol{K}_{\mathrm{M}}\left[\boldsymbol{X} ; \mathcal{K}_{d}(K)\right]=\boldsymbol{K}_{\mathrm{M}}\left[\boldsymbol{X} ; \mathcal{K}_{d}^{\mathrm{C}}(K ; r)\right]$ since $\mathcal{K}_{d}^{\mathrm{C}}(K ; r) \subseteq \mathcal{K}_{d}(K)$. We check these observations numerically by calculating the corresponding estimates. 
(a)

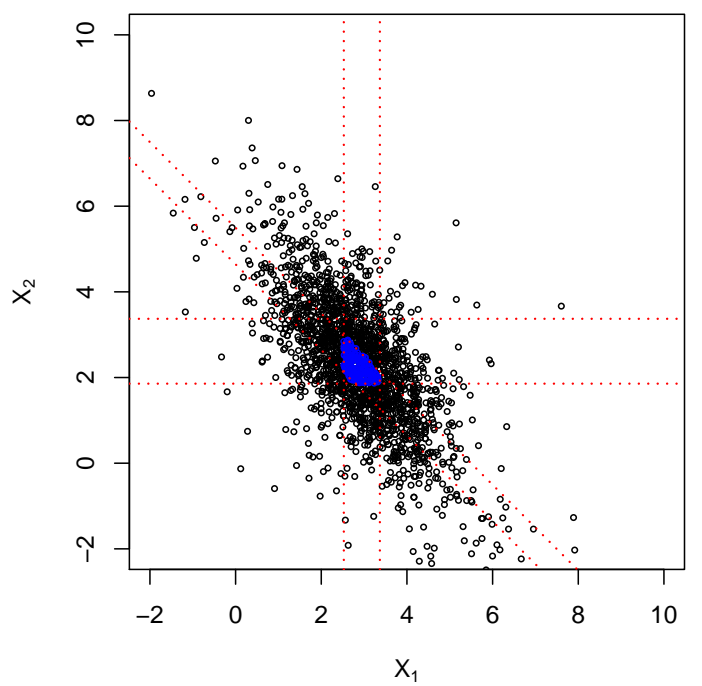

(b)

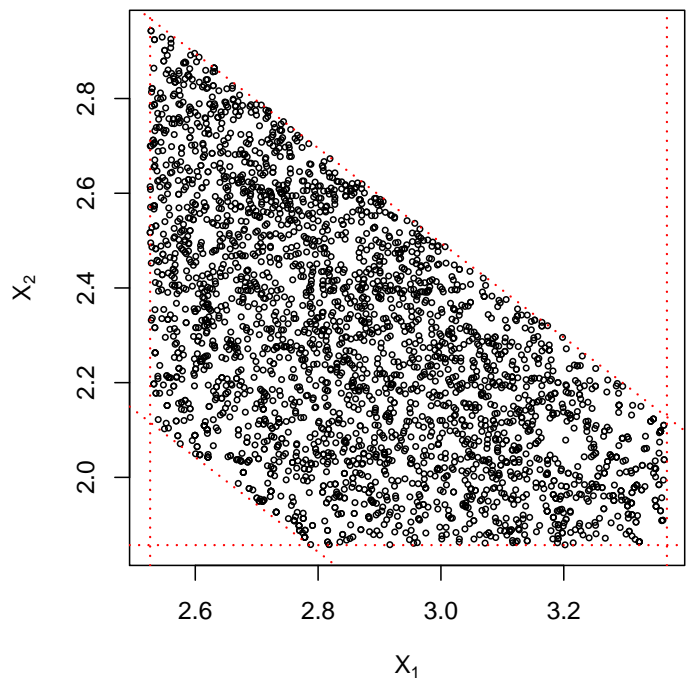

Figure 4: Scatter plots of (a) MC samples from $\boldsymbol{X}^{\prime} \mid\left\{\boldsymbol{X} \in \mathcal{K}_{d}(K, \delta)\right\}$ (black) and $\boldsymbol{X}^{\prime} \mid\left\{\boldsymbol{X} \in \mathcal{K}_{d}^{\mathrm{C}}(K, \delta ; r)\right\}$ (blue), and of (b) MCMC samples from $\boldsymbol{X}^{\prime} \mid\left\{\boldsymbol{X} \in \mathcal{K}_{d}^{\mathrm{C}}(K ; r)\right\}$ (black) where $\boldsymbol{X} \sim t_{\nu}\left(\mathbf{0}_{d}, P\right)$ with $d=3, \nu=5$ and $P=\left(\rho_{i, j}\right)$ being a correlation matrix with $\rho_{1,2}=\rho_{2,3}=1 / 3$ and $\rho_{1,3}=2 / 3, r(\boldsymbol{\lambda})=\operatorname{VaR}_{p}\left(\boldsymbol{\lambda}^{\top} \boldsymbol{X}\right)$ with $p=0.99$ for $\boldsymbol{\lambda} \in\{0,1\}^{3}, K=r\left(\mathbf{1}_{3}\right)$ and $\delta=0.001$. Red lines indicate $\left\{\boldsymbol{x}^{\prime} \in \mathbb{R}^{2}: \boldsymbol{\lambda}^{\top}\left(\boldsymbol{x}^{\prime}, K-\mathbf{1}_{2}^{\top} \boldsymbol{x}^{\prime}\right)=r(\boldsymbol{\lambda})\right\}$ for $\boldsymbol{\lambda} \in\{0,1\}^{3}$.

Table 4 summarizes the MC and MCMC estimates and standard errors of the Euler and maximum likelihood allocations on $\mathcal{K}_{d}(K)$ and those on the atomic core $\mathcal{K}_{d}^{\mathrm{C}}(K ; r)$. MC and MCMC estimates are calculated based on the samples in Figure 4 (a), and based on those in Figure 4 (b), respectively. As expected by theory, the MC estimates of $\boldsymbol{K}_{\mathrm{M}}\left[\boldsymbol{X} ; \mathcal{K}_{d}(K)\right]$ and $\mathbb{E}\left[\boldsymbol{X} \mid\left\{\boldsymbol{X} \in \mathcal{K}_{d}(K)\right\}\right]$ were close to each other. We can also observe that the MC and MCMC estimates are close to each other for all the estimators. The standard errors of the MCMC estimator of $\mathbb{E}\left[\boldsymbol{X} \mid\left\{\boldsymbol{X} \in \mathcal{K}_{d}^{\mathrm{C}}(K ; r)\right\}\right]$ were smaller than those of the $\mathrm{MC}$ estimator because of sample efficiency. Provided that $\hat{\boldsymbol{K}}_{\mathrm{M}}^{\mathrm{MC}}\left[\boldsymbol{X} ; \mathcal{K}_{d}(K)\right]$ belongs to the core $\mathcal{K}_{d}^{\mathrm{C}}(K ; r)$, we expect an estimate of $\boldsymbol{K}_{\mathrm{M}}\left[\boldsymbol{X} ; \mathcal{K}_{d}^{\mathrm{C}}(K ; r)\right]$ to be close to $\hat{\boldsymbol{K}}_{\mathrm{M}}^{\mathrm{MC}}\left[\boldsymbol{X} ; \mathcal{K}_{d}(K)\right]$. Although this was the case for both of the MC and MCMC estimates of $\boldsymbol{K}_{\mathrm{M}}\left[\boldsymbol{X} ; \mathcal{K}_{d}^{\mathrm{C}}(K ; r)\right]$, the MCMC estimate was slightly closer to $\hat{\boldsymbol{K}}_{\mathrm{M}}^{\mathrm{MC}}\left[\boldsymbol{X} ; \mathcal{K}_{d}(K)\right]$ than the MC estimate. Consequently, the MCMC estimator of $\boldsymbol{K}_{\mathrm{M}}\left[\boldsymbol{X} ; \mathcal{K}_{d}^{\mathrm{C}}(K ; r)\right]$ can be said to be less biased than the MC estimator. 
Table 4: Monte Carlo (MC) and Markov chain Monte Carlo (MCMC) estimates and standard errors of the Euler and maximum likelihood allocations on $\mathcal{K}_{d}(K)$ and those on the atomic core $\mathcal{K}_{d}^{\mathrm{C}}(K ; r)$. The MC sample size of $\boldsymbol{X}$ is $N_{\mathrm{MC}}=10^{6}$ and the MCMC sample size of $\boldsymbol{X} \mid\left\{\boldsymbol{X} \in \mathcal{K}_{d}^{\mathrm{C}}(K ; r)\right\}$ is $N_{\mathrm{MCMC}}=10^{4}$.

\begin{tabular}{lccccccc}
\hline & \multicolumn{3}{c}{ Estimator } & & \multicolumn{3}{c}{ Standard error } \\
\cline { 2 - 3 } & $X_{1}$ & $X_{2}$ & $X_{3}$ & & $X_{1}$ & $X_{2}$ & $X_{3}$ \\
\hline$\hat{\mathbb{E}}^{\mathrm{MC}}\left[\boldsymbol{X} \mid\left\{\boldsymbol{X} \in \mathcal{K}_{d}(K)\right\}\right]$ & 2.865 & 2.310 & 2.846 & & 0.026 & 0.034 & 0.026 \\
$\hat{\boldsymbol{K}}_{\mathrm{M}}^{\mathrm{MC}}\left[\boldsymbol{X} ; \mathcal{K}_{d}(K)\right]$ & 2.861 & 2.366 & 2.793 & - & - & - \\
$\hat{\mathbb{E}}^{\mathrm{MC}}\left[\boldsymbol{X} \mid\left\{\boldsymbol{X} \in \mathcal{K}_{d}^{\mathrm{C}}(K ; r)\right\}\right]$ & 2.852 & 2.267 & 2.903 & & 0.016 & 0.019 & 0.016 \\
$\hat{\boldsymbol{K}}_{\mathrm{M}}^{\mathrm{MC}}\left[\boldsymbol{X} ; \mathcal{K}_{d}^{\mathrm{C}}(K ; r)\right]$ & 2.838 & 2.262 & 2.920 & - & - & - \\
$\hat{\mathbb{E}}^{\mathrm{MCMC}}\left[\boldsymbol{X} \mid\left\{\boldsymbol{X} \in \mathcal{K}_{d}^{\mathrm{C}}(K ; r)\right\}\right]$ & 2.876 & 2.269 & 2.877 & & 0.002 & 0.003 & 0.002 \\
$\hat{\boldsymbol{K}}_{\mathrm{M}}^{\mathrm{MCMC}}\left[\boldsymbol{X} ; \mathcal{K}_{d}^{\mathrm{C}}(K ; r)\right]$ & 2.866 & 2.283 & 2.871 & - & - & - \\
\hline
\end{tabular}

